\title{
Copper-Catalyzed Hydroxylation of (Hetero)aryl Halides under Mild Conditions
}

Shanghua Xia, Lu Gan, Kailiang Wang, Zheng Li and Dawei Ma*

Interdisciplinary Research Center on Biology and Chemistry, Shanghai Institute of Organic Chemistry, Chinese Academy of Sciences, 354 Fenglin Lu, Shanghai 200032, China

E-mail: $\underline{\text { madw@mail.sioc.ac.cn }}$

\section{Supporting Information}

Table of contents

1. General procedure for the preparation of ligands---------------------------------S2

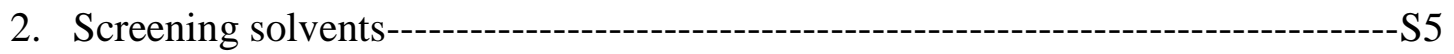

3. General procedure for the $\mathrm{Cu}$-catalyzed synthesis of phenols and hydroxylated

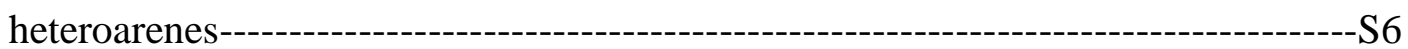

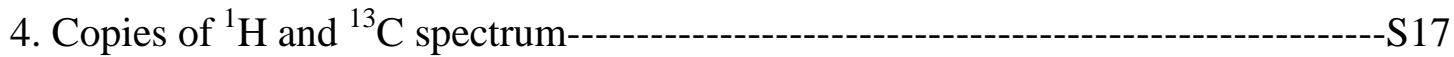




\section{General procedures for the preparation of ligands}

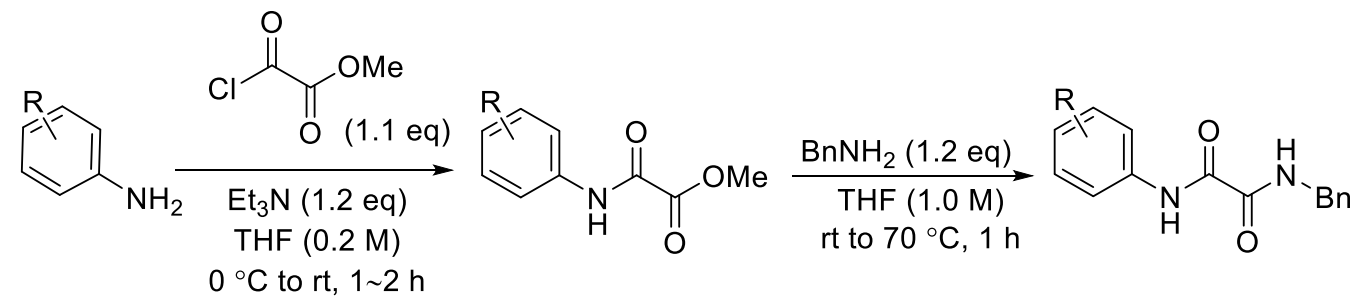

General procedure A: To a solution of the corresponding aniline (1.0 eq.) in THF $(0.5 \mathrm{M})$ was added $\mathrm{Et}_{3} \mathrm{~N}$ (1.2 eq.). Mono-methyl oxalyl chloride (1.1 eq.) was then added to the solution slowly under cooling with ice-water bath. After the resulting mixture was stirred at room temperature for $1-2 \mathrm{~h}$, the mixture was washed with the same volume of water. The organic phase was dried over $\mathrm{Na}_{2} \mathrm{SO}_{4}$ and evaporated. The crude product was purified with silica gel chromatography to afford the corresponding methyl $N$-aryloxamate.

To a magnetically stirred solution of the above monoamide $(10 \mathrm{mmol})$ in THF $(1.0 \mathrm{M})$ was added $\mathrm{BnNH}_{2}(1.2$ eq.) at room temperature. After the resulting mixture was stirred under $70{ }^{\circ} \mathrm{C}$ for $1 \mathrm{~h}$, it was cooled to room temperature in the air and then to $-18{ }^{\circ} \mathrm{C}$ in refrigerator. In most cases, the products would precipitate out as crystals. If no precipitate appeared, hexane was added to the mixture until the products precipitated out. The mixture was filtered, and the solids were collected and washed with small amount of cold diethyl ether to afford the corresponding $N$-benzyl- $N$ aryloxalamide. They were pure enough to be used. (Note: $\mathrm{BnNH}_{2}$ can be replaced by other aliphatic amines to afford the corresponding $N$-alkyl- $N$-aryloxalamide.)

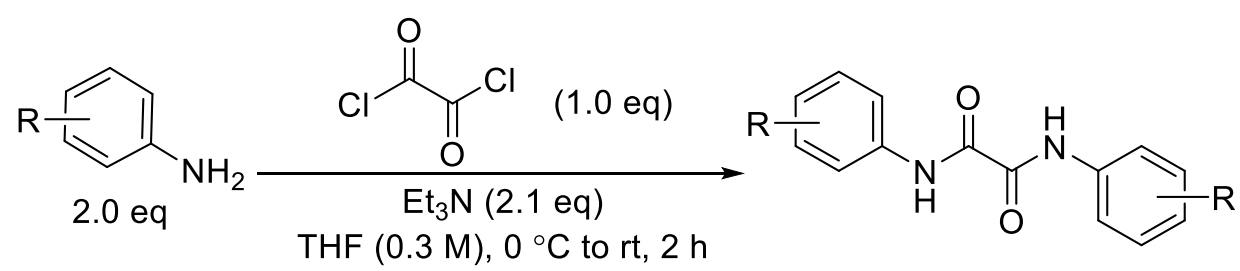

General procedure B: To a solution of the corresponding aniline (2.0 eq.) in THF $(0.3 \mathrm{M})$ was added $\mathrm{Et}_{3} \mathrm{~N}$ (2.1 eq.). Oxalyl chloride (1.0 eq.) was then added to the solution slowly at $0{ }^{\circ} \mathrm{C}$. After the resulting mixture was stirred at room temperature for $2 \mathrm{~h}$, it was concentrated in vacuo to remove the solvent. Water was added to the 
resulting residue to dissolve $\mathrm{Et}_{3} \mathrm{~N} \cdot \mathrm{HCl}$. The slurry was filtered and the solid on filter paper was washed with water and cold diethyl ether. These solids were dried in vacuo to afford the corresponding $N, N^{\prime}$-diaryloxalamide. They were pure enough to be used.<smiles>Cc1ccc(NC(=O)C(=O)NCc2ccccc2)c(P)c1</smiles>

L1

Following the general procedures $\mathrm{A}, \mathbf{L 1}^{1}$ was prepared from 4-methyl-2phenylaniline, methyl oxalyl chloride and $\mathrm{BnNH}_{2}$ in two steps as white solid in $85 \%$ overall yield. ${ }^{1} \mathrm{H}$ NMR $\left(400 \mathrm{MHz}, \mathrm{CDCl}_{3}\right) \delta 9.46(\mathrm{~s}, 1 \mathrm{H}), 8.30-8.29(\mathrm{~d}, J=6.8 \mathrm{~Hz}$, 1H), 7.81 (s, 1H), 7.53-7.50 (m, 2H), 7.45-7.42 (m, 1H), 7.40-7.38 (m, 1H), 7.35-7.32 (m, 2H), 7.30-7.28 (m, 3H), 7.22-7.20 (d, $J=6.8 \mathrm{~Hz}, 1 \mathrm{H}), 7.14(\mathrm{~d}, J=1.2 \mathrm{~Hz}, 1 \mathrm{H})$, 4.48-4.47 (d, $J=4.8 \mathrm{~Hz}, 2 \mathrm{H}), 2.38(\mathrm{~s}, 3 \mathrm{H}) ;{ }^{13} \mathrm{C}$ NMR (100 MHz, DMSO- $\left.d_{6}\right) \delta 159.8$, 157.9, 138.5, 137.9, 135.1, 134.5, 131.1, 130.8, 128.8 (2C), 128.79 (2C), 128.6, 128.3 (2C), 127.7, 127.4 (2C), 127.0, 122.7, 42.6, 20.5.<smiles>Cc1ccc(NC(=O)C(C)(C)C)c(P)c1</smiles>

L2

Following the general procedure $\mathrm{B}, \mathbf{L 2}^{2}$ was prepared from 2-phenyl-4methylaniline and oxalyl chloride as a white solid in $91 \%$ yield. ${ }^{1} \mathrm{H}$ NMR $(400 \mathrm{MHz}$, $\left.\mathrm{CDCl}_{3}\right) \delta 9.47(\mathrm{~s}, 2 \mathrm{H}), 8.24(\mathrm{~d}, J=8.3 \mathrm{~Hz}, 2 \mathrm{H}), 7.55-7.48(\mathrm{~m}, 4 \mathrm{H}), 7.47-7.41(\mathrm{~m}, 2 \mathrm{H})$, 7.40-7.35 (m, 4H), $7.17(\mathrm{~d}, J=8.6 \mathrm{~Hz}, 2 \mathrm{H}), 7.12(\mathrm{~s}, 2 \mathrm{H}), 2.36(\mathrm{~s}, 6 \mathrm{H}),{ }^{13} \mathrm{C} \mathrm{NMR}(100$ $\left.\mathrm{MHz}, \mathrm{CDCl}_{3}\right) \delta 157.4,137.6,135.1,133.0,131.1,130.9,129.4,129.3,129.1,128.4$, $120.4,21.1$.<smiles>CC(C)(C)C(=O)Nc1ccccc1Oc1ccccc1</smiles>

Following the general procedure $\mathrm{B}, \mathbf{L 3}^{2}$ was prepared from 2-phenoxyaniline and oxalyl chloride as a white solid in $85 \%$ yield. ${ }^{1} \mathrm{H} \mathrm{NMR}\left(400 \mathrm{MHz}, \mathrm{CDCl}_{3}\right) \delta 9.97(\mathrm{~s}$, 
2H), $8.47(\mathrm{dd}, J=8.0,1.8 \mathrm{~Hz}, 2 \mathrm{H}), 7.42-7.34(\mathrm{~m}, 4 \mathrm{H}), 7.20-7.10(\mathrm{~m}, 5 \mathrm{H}), 7.10-7.05$ $(\mathrm{m}, 5 \mathrm{H}), 6.90(\mathrm{dd}, J=8.0,1.6 \mathrm{~Hz}, 2 \mathrm{H}) ;{ }^{13} \mathrm{C} \mathrm{NMR}\left(100 \mathrm{MHz}, \mathrm{CDCl}_{3}\right) \delta 157.5,156.2$, $146.9,130.1,128.1,125.6,124.3,123.9,120.6,119.2,117.8$.<smiles>Cc1cccc(C)c1NC(=O)CC(C)C</smiles>

L4

Following the general procedure B, $\mathbf{L} \mathbf{4}^{2}$ was prepared from 2,6-dimethylaniline and oxalyl chloride as a white solid in $88 \%$ yield. ${ }^{1} \mathrm{H}$ NMR (400 MHz, DMSO- $\left.d_{6}\right) \delta$ $10.29(\mathrm{~s}, 2 \mathrm{H}), 7.18-7.08(\mathrm{~m}, 6 \mathrm{H}), 2.18(\mathrm{~s}, 12 \mathrm{H}) ;{ }^{13} \mathrm{C}$ NMR $\left(100 \mathrm{MHz}, \mathrm{DMSO}-d_{6}\right) \delta$ $158.9,135.1,134.3,127.8,127.0,18.0$.<smiles>CC(=O)Nc1c(C)cc(O)cc1C</smiles>

L5

Following the general procedure B, L5 was prepared from 4-amino-3,5dimethylphenol and oxalyl chloride as a white solid in $87 \%$ yield. ${ }^{1} \mathrm{H}$ NMR (400 MHz, DMSO- $\left.d_{6}\right) \delta 9.96(\mathrm{~s}, 2 \mathrm{H}), 9.25(\mathrm{~s}, 2 \mathrm{H}), 6.52(\mathrm{~s}, 4 \mathrm{H}), 2.08(\mathrm{~s}, 12 \mathrm{H}) ;{ }^{13} \mathrm{C}$ NMR $(100$ MHz, DMSO- $\left.d_{6}\right) \delta 159.3,155.9,136.1,125.6,114.3,18.2$. ESI-HRMS: $m / z$ calcd for $\mathrm{C}_{18} \mathrm{H}_{19} \mathrm{~N}_{2} \mathrm{O}_{4}(\mathrm{M}-\mathrm{H})^{-} 327.1345$, found: 327.1348<smiles>COc1cc(C)c(NC(C)=O)c(C(C)(C)C)c1</smiles>

Following the general procedure B, $\mathbf{L 6}^{2}$ was prepared from 4-methoxy-2,6dimethylaniline and oxalyl chloride as a pale yellow solid in $93 \%$ yield. ${ }^{1} \mathrm{H}$ NMR (400 MHz, DMSO- $\left.d_{6}\right) \delta 10.09(\mathrm{~s}, 2 \mathrm{H}), 6.69(\mathrm{~s}, 4 \mathrm{H}), 3.74(\mathrm{~s}, 6 \mathrm{H}), 2.13(\mathrm{~s}, 12 \mathrm{H}) ;{ }^{13} \mathrm{C} \mathrm{NMR}$ $\left(100 \mathrm{MHz}, \mathrm{DMSO}-d_{6}\right) \delta 159.2,157.7,136.3,127.0,112.9,55.1,18.2$. 


\section{Screening of solvents}

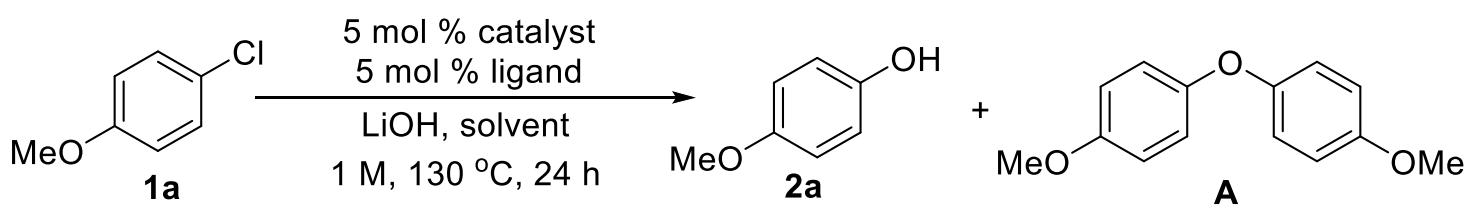

\begin{tabular}{|c|c|c|c|c|c|c|c|c|c|}
\hline $\begin{array}{c}\mathrm{DMSO} / \mathrm{H}_{2} \mathrm{O} \\
(\mathrm{ml} / \mathrm{ml})\end{array}$ & $0.2 / 1.8$ & $0.4 / 1.6$ & $0.6 / 1.4$ & $0.8 / 1.2$ & $1.0 / 1.0$ & $1.2 / 0.8$ & $1.4 / 0.6$ & $1.6 / 0.4$ & $1.8 / 0.2$ \\
\hline $\mathbf{1 a}(\%)$ & 83 & 83 & 76 & 62 & 41 & 34 & 20 & 5 & 18 \\
\hline $\mathbf{2 a}(\%)$ & 10 & 9 & 16 & 29 & 46 & 55 & 65 & 87 & 60 \\
\hline $\mathbf{A}(\%)$ & 0 & 0 & 0 & 0 & 0 & 0 & 2 & 2 & 14 \\
\hline
\end{tabular}

\begin{tabular}{|c|c|c|c|c|}
\hline Solvent $(\mathrm{v} / \mathrm{v}=4: 1)$ & $\mathrm{DMSO} / \mathrm{H}_{2} \mathrm{O}$ & $\mathrm{DMA} / \mathrm{H}_{2} \mathrm{O}$ & $\mathrm{NMP} / \mathrm{H}_{2} \mathrm{O}$ & Dioxane $/ \mathrm{H}_{2} \mathrm{O}$ \\
\hline $\mathbf{1 a}(\%)$ & 5 & 83 & 48 & 96 \\
\hline $\mathbf{2 a}(\%)$ & 87 & 7 & 35 & 0 \\
\hline $\mathbf{A}(\%)$ & 2 & 0 & 0 & 0 \\
\hline
\end{tabular}




\section{General procedure for the Cu-catalyzed synthesis of phenols and hydroxylated}

heteroarenes
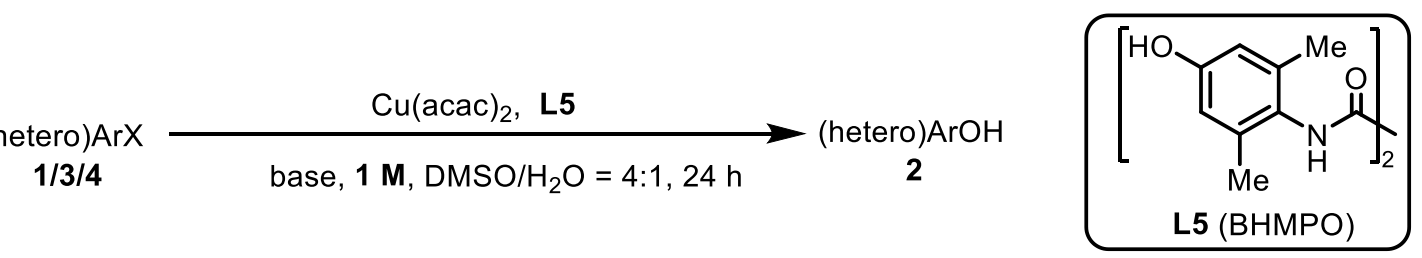

$\mathbf{X}=\mathbf{C l}$

The (hetero)aryl chloride (2.0 mmol), $\mathrm{Cu}(\mathrm{acac})_{2}(0.1 \mathrm{mmol}, 26.2 \mathrm{mg}), \mathrm{LiOH} \cdot \mathrm{H}_{2} \mathrm{O}$ (4.2 mmol, $176 \mathrm{mg})$ and ligand $\mathbf{L 5}(0.1 \mathrm{mmol}, 32.8 \mathrm{mg})$ were placed into a Schlenk tube $(10 \mathrm{~mL})$ with a magnetic stir bar. The reaction vessel was evacuated and backfilled with argon three times, then DMSO $(1.6 \mathrm{~mL})$ and degassed water $(0.4 \mathrm{ml})$ were added under a positive argon pressure (Note: for liquid substrates, they were added after the tube was backfilled with argon). The reaction mixture was heated at $130{ }^{\circ} \mathrm{C}$ for $24 \mathrm{~h}$ under vigorous stirring. The cooled solution was acidified with $2 \mathrm{~N}$ $\mathrm{HCl}$, then diluted with ethyl acetate and washed with brine. The organic phase was dried over $\mathrm{Na}_{2} \mathrm{SO}_{4}$ and concentrated in vacuo. The residue was purified by silica gel flash chromatography to afford the corresponding phenols or hydroxylated heteroarenes.

$\mathbf{X}=\mathbf{B r}$

The (hetero)aryl bromide (4.0 mmol), $\mathrm{Cu}(\mathrm{acac})_{2}(0.02 \mathrm{mmol}, 5.3 \mathrm{mg}), \mathrm{LiOH} \cdot \mathrm{H}_{2} \mathrm{O}$ (8.4 mmol, $352 \mathrm{mg})$ and ligand $\mathbf{L 5}(0.02 \mathrm{mmol}, 6.6 \mathrm{mg})$ were placed into a Schlenk tube $(25 \mathrm{~mL})$ with a magnetic stir bar. The reaction vessel was evacuated and backfilled with argon three times, then DMSO $(3.2 \mathrm{~mL})$ and degassed water $(0.8 \mathrm{ml})$ were added under a positive argon pressure (Note: for liquid substrates, they were added after the tube was backfilled with argon). The reaction mixture was heated at $80{ }^{\circ} \mathrm{C}$ for $24 \mathrm{~h}$ under vigorous stirring. The cooled solution was acidified with $2 \mathrm{~N}$ $\mathrm{HCl}$, then diluted with ethyl acetate and washed with brine. The organic phase was dried over $\mathrm{Na}_{2} \mathrm{SO}_{4}$ and concentrated in vacuo. The residue was purified by silica gel flash chromatography to afford the corresponding phenols or hydroxylated heteroarenes. 
$\mathbf{X}=\mathbf{I}$

The (hetero)aryl iodide $(4.0 \mathrm{mmol}), \mathrm{Cu}(\mathrm{acac})_{2}(0.02 \mathrm{mmol}, 5.3 \mathrm{mg}), \mathrm{KOH}(12$ mmol, $792 \mathrm{mg})$ and ligand $\mathbf{L 5}(0.02 \mathrm{mmol}, 6.6 \mathrm{mg})$ were placed into a Schlenk tube $(25 \mathrm{~mL})$ with a magnetic stir bar. The reaction vessel was evacuated and backfilled with argon three times, then DMSO $(3.2 \mathrm{~mL})$ and degassed water $(0.8 \mathrm{ml})$ were added under a positive argon pressure (Note: for liquid substrates, they were added after the tube was backfilled with argon). The reaction mixture was heated at $60{ }^{\circ} \mathrm{C}$ for $24 \mathrm{~h}$ under vigorous stirring. The cooled solution was acidified with $2 \mathrm{~N} \mathrm{HCl}$, then diluted with ethyl acetate and washed with brine. The organic phase was dried over $\mathrm{Na}_{2} \mathrm{SO}_{4}$ and concentrated in vacuo. The residue was purified by silica gel flash chromatography to afford the corresponding phenols or hydroxylated heteroarenes.

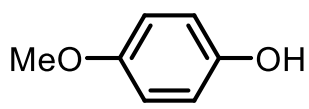

2a

2a: white brown solid; ${ }^{1} \mathrm{H}$ NMR (400 $\left.\mathrm{MHz}, \mathrm{CDCl}_{3}\right) \delta$ 6.87-6.69 (m, 4H), $5.05(\mathrm{~s}$, 1H), 3.77 (s, 3H); ${ }^{13} \mathrm{C} \mathrm{NMR}\left(100 \mathrm{MHz}, \mathrm{CDCl}_{3}\right) \delta 153.8,149.7,116.2,115.0,56.0$.

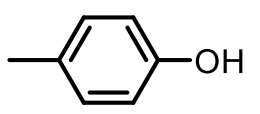

2b

2b: pale yellow oil; ${ }^{1} \mathrm{H}$ NMR $\left(400 \mathrm{MHz}, \mathrm{CDCl}_{3}\right) \delta 7.04(\mathrm{~d}, J=8.1 \mathrm{~Hz}, 2 \mathrm{H}), 6.74(\mathrm{~d}$, $J=8.4 \mathrm{~Hz}, 2 \mathrm{H}), 4.94(\mathrm{~s}, 1 \mathrm{H}), 2.28(\mathrm{~s}, 3 \mathrm{H}) ;{ }^{13} \mathrm{C} \mathrm{NMR}\left(100 \mathrm{MHz}, \mathrm{CDCl}_{3}\right) \delta 153.4$, $130.2,130.1,115.2,20.6$.<smiles>CCCOc1ccc(O)cc1</smiles>

2c

2c: white solid; ${ }^{1} \mathrm{H}$ NMR (400 MHz, $\left.\mathrm{CDCl}_{3}\right) \delta$ 6.90-6.82 (m, 2H), 6.74-6.66 (m, 2H), $4.90(\mathrm{~s}, 1 \mathrm{H}), 1.30(\mathrm{~s}, 9 \mathrm{H}) ;{ }^{13} \mathrm{C} \mathrm{NMR}\left(100 \mathrm{MHz}, \mathrm{CDCl}_{3}\right) \delta 151.9,148.6,125.7,115.5$, $78.4,28.8$.

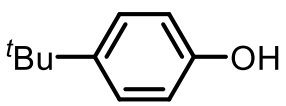

2d

2d: pale pink solid; ${ }^{1} \mathrm{H}$ NMR $\left(400 \mathrm{MHz}, \mathrm{CDCl}_{3}\right) \delta$ 7.28-7.21 (m, 2H), 6.80-6.72 (m, 2H), 5.09 (s, 1H), $1.28(\mathrm{~s}, 9 \mathrm{H}) ;{ }^{13} \mathrm{C} \mathrm{NMR}\left(100 \mathrm{MHz}, \mathrm{CDCl}_{3}\right) \delta$ 153.1, 143.7, 126.6, 114.9, 34.2, 31.7 . 


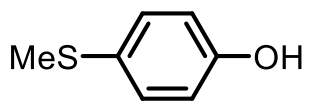

$2 e$

2e: white solid; ${ }^{1} \mathrm{H}$ NMR (400 MHz, $\left.\mathrm{CDCl}_{3}\right) \delta$ 7.25-7.20 (m, 2H), 6.81-6.76 (m, 2H), $5.09(\mathrm{~s}, 1 \mathrm{H}), 2.44(\mathrm{~s}, 3 \mathrm{H}) ;{ }^{13} \mathrm{C}$ NMR $\left(100 \mathrm{MHz}, \mathrm{CDCl}_{3}\right) \delta 154.2,130.5,129,0,116.2$, 18.2 .

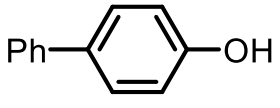

$2 \mathbf{f}$

2f: white solid; ${ }^{1} \mathrm{H}$ NMR $\left(400 \mathrm{MHz}, \mathrm{DMSO}-d_{6}\right) \delta 9.55(\mathrm{~s}, 1 \mathrm{H}), 7.56(\mathrm{~d}, J=7.4 \mathrm{~Hz}$, 2H), $7.48(\mathrm{~d}, J=8.6 \mathrm{~Hz}, 2 \mathrm{H}), 7.40(\mathrm{t}, J=7.7 \mathrm{~Hz}, 2 \mathrm{H}), 7.27(\mathrm{t}, J=7.3 \mathrm{~Hz}, 1 \mathrm{H}), 6.87$ $(\mathrm{d}, J=8.6 \mathrm{~Hz}, 2 \mathrm{H}) ;{ }^{13} \mathrm{C}$ NMR $\left(100 \mathrm{MHz}, \mathrm{DMSO}-d_{6}\right) \delta 157.2,140.2,131.0,128.8$, $127.7,126.3,126.0,115.7$.<smiles>NC(=O)c1ccc(O)cc1</smiles>

2g: pale yellow solid; ${ }^{1} \mathrm{H}$ NMR (400 MHz, DMSO- $\left.d_{6}\right) \delta 9.95(\mathrm{~s}, 1 \mathrm{H}), 7.80-7.65(\mathrm{~m}$, $3 \mathrm{H}), 7.07(\mathrm{~s}, 1 \mathrm{H}), 6.83-6.74(\mathrm{~m}, 2 \mathrm{H}) ;{ }^{13} \mathrm{C} \mathrm{NMR}\left(100 \mathrm{MHz}, \mathrm{DMSO}-d_{6}\right) \delta 167.7$, $160.2,129.5,125.0,114.7$

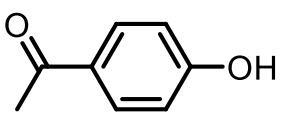

$2 \mathrm{~h}$

2h: pale yellow solid; ${ }^{1} \mathrm{H}$ NMR $\left(400 \mathrm{MHz}, \mathrm{CDCl}_{3}\right) \delta 8.67(\mathrm{~s}, 1 \mathrm{H}), 7.92(\mathrm{~d}, J=8.7$ $\mathrm{Hz}, 2 \mathrm{H}), 6.98(\mathrm{~d}, J=8.7 \mathrm{~Hz}, 2 \mathrm{H}), 2.59(\mathrm{~s}, 3 \mathrm{H}) ;{ }^{13} \mathrm{C} \mathrm{NMR}\left(100 \mathrm{MHz}, \mathrm{CDCl}_{3}\right) \delta 199.4$, 162.0, 131.5, 129.3, 115.8, 26.4.

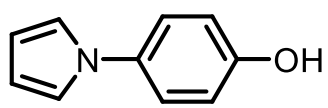

2i

2i: pale pink solid; ${ }^{1} \mathrm{H}$ NMR $\left(400 \mathrm{MHz}, \mathrm{CDCl}_{3}\right) \delta$ 7.26-7.20 (m, 2H), $6.98(\mathrm{t}, J=2.2$ $\mathrm{Hz}, 2 \mathrm{H}), 6.86-6.79(\mathrm{~m}, 2 \mathrm{H}), 6.32(\mathrm{t}, J=2.2 \mathrm{~Hz}, 2 \mathrm{H}), 5.11(\mathrm{~s}, 1 \mathrm{H}) ;{ }^{13} \mathrm{C}$ NMR $(100$ $\left.\mathrm{MHz}, \mathrm{CDCl}_{3}\right) \delta 153.6,134.8,122.5,119.9,116.2,110.0$.

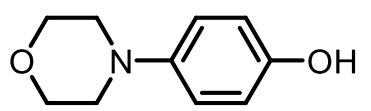

2j 
2j: yellowish-brown solid; ${ }^{1} \mathrm{H}$ NMR (400 MHz, DMSO- $\left.d_{6}\right) \delta 8.83(\mathrm{~s}, 1 \mathrm{H}), 6.72(\mathrm{dd}$, $J=43.0,8.7 \mathrm{~Hz}, 4 \mathrm{H}), 3.80-3.61(\mathrm{~m}, 4 \mathrm{H}), 2.99-2.83(\mathrm{~m}, 4 \mathrm{H}) ;{ }^{13} \mathrm{C} \mathrm{NMR}(100 \mathrm{MHz}$, DMSO- $\left.d_{6}\right) \delta 151.1,144.2,117.4,115.5,66.3,50.2$.

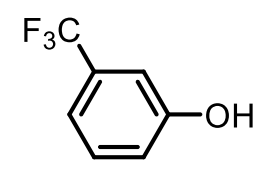

2k

2k: pale yellow oil; ${ }^{1} \mathrm{H}$ NMR $\left(400 \mathrm{MHz}, \mathrm{CDCl}_{3}\right) \delta 7.85(\mathrm{~s}, 1 \mathrm{H}), 7.30(\mathrm{t}, J=8.2 \mathrm{~Hz}$, 1H), 7.16-7.11 (m, 2H), 7.07-7.01 (m, 1H); $\left.{ }^{13} \mathrm{C} \mathrm{NMR} \mathrm{(100} \mathrm{MHz,} \mathrm{CDCl}_{3}\right) \delta$ 155.6, $132.1(\mathrm{q}, J=32.4 \mathrm{~Hz}), 130.3,118.9,117.7(\mathrm{q}, J=3.9 \mathrm{~Hz}), 112.4(\mathrm{q}, J=3.8 \mathrm{~Hz})$.

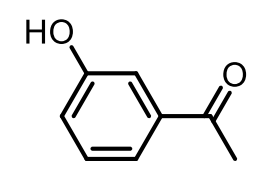

2l

21: white brown solid; ${ }^{1} \mathrm{H} \mathrm{NMR}\left(400 \mathrm{MHz}, \mathrm{CDCl}_{3}\right) \delta$ 7.56-7.48 (m, 2H), $7.34(\mathrm{t}, J=$ $7.8 \mathrm{~Hz}, 1 \mathrm{H}), 7.10(\mathrm{ddd}, J=8.1,2.5,0.9 \mathrm{~Hz}, 1 \mathrm{H}), 6.43(\mathrm{~s}, 1 \mathrm{H}), 2.60(\mathrm{~s}, 3 \mathrm{H}) ;{ }^{13} \mathrm{C}$ NMR $\left(100 \mathrm{MHz}, \mathrm{CDCl}_{3}\right) \delta 199.7,156.6,138.4,130.0,121.2,121.1,114.9,26.9$.

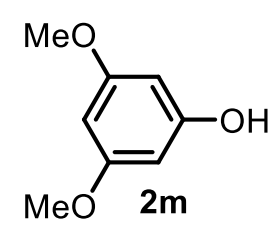

2m: colorless oil; ${ }^{1} \mathrm{H}$ NMR $\left(400 \mathrm{MHz}, \mathrm{CDCl}_{3}\right) \delta 6.08(\mathrm{t}, J=2.1 \mathrm{~Hz}, 1 \mathrm{H}), 6.04(\mathrm{~d}, J$ $=2.2 \mathrm{~Hz}, 2 \mathrm{H}), 5.83(\mathrm{~s}, 1 \mathrm{H}), 3.74(\mathrm{~s}, 6 \mathrm{H}) ;{ }^{13} \mathrm{C} \mathrm{NMR}\left(100 \mathrm{MHz}, \mathrm{CDCl}_{3}\right) \delta 161.7$, 157.6, 94.5, 93.3, 55.5.

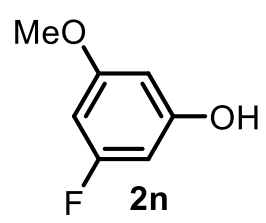

2n: pale yellow solid; ${ }^{1} \mathrm{H}$ NMR (400 MHz, $\left.\mathrm{CDCl}_{3}\right) \delta 6.24(\mathrm{dt}, J=10.7,2.2 \mathrm{~Hz}, 1 \mathrm{H})$, 6.22-6.16 (m, 2H), $5.66(\mathrm{~s}, 1 \mathrm{H}), 3.76(\mathrm{~s}, 3 \mathrm{H}) ;{ }^{13} \mathrm{C} \mathrm{NMR}\left(100 \mathrm{MHz}, \mathrm{CDCl}_{3}\right) \delta 164.3$ $(\mathrm{d}, J=243.7 \mathrm{~Hz}), 161.6(\mathrm{~d}, J=13.8 \mathrm{~Hz}), 157.3(\mathrm{~d}, J=14.4 \mathrm{~Hz}), 97.7(\mathrm{~d}, J=3.0 \mathrm{~Hz})$, 
$96.1(\mathrm{~d}, J=25.2 \mathrm{~Hz}), 94.6(\mathrm{~d}, J=25.5 \mathrm{~Hz}), 55.7$; ESI-HRMS: $\mathrm{m} / \mathrm{z}$ calcd for $\mathrm{C}_{7} \mathrm{H}_{6} \mathrm{FO}_{2}(\mathrm{M}-\mathrm{H})^{-}$141.0352, found: 141.0357.

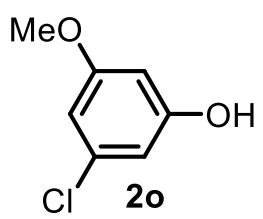

2o: white solid; ${ }^{1} \mathrm{H}$ NMR $\left(400 \mathrm{MHz}, \mathrm{CDCl}_{3}\right) \delta 6.51(\mathrm{t}, J=1.9 \mathrm{~Hz}, 1 \mathrm{H}), 6.46(\mathrm{t}, J=$ $1.9 \mathrm{~Hz}, 1 \mathrm{H}), 6.30(\mathrm{t}, J=2.2 \mathrm{~Hz}, 1 \mathrm{H}), 5.63(\mathrm{~s}, 1 \mathrm{H}), 3.76(\mathrm{~s}, 3 \mathrm{H}) ;{ }^{13} \mathrm{C} \mathrm{NMR}(100 \mathrm{MHz}$, $\left.\mathrm{CDCl}_{3}\right) \delta 161.3,157.0,135.5,108.8,107.3,100.5,55.7$.

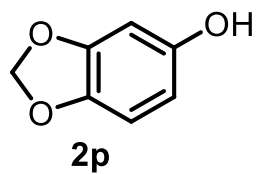

2p: white solid; ${ }^{1} \mathrm{H}$ NMR $\left(400 \mathrm{MHz}, \mathrm{CDCl}_{3}\right) \delta 6.65(\mathrm{~d}, J=8.3 \mathrm{~Hz}, 1 \mathrm{H}), 6.43(\mathrm{~d}, J=$ $2.5 \mathrm{~Hz}, 1 \mathrm{H}), 6.25(\mathrm{dd}, J=8.3,2.5 \mathrm{~Hz}, 1 \mathrm{H}), 5.91(\mathrm{~s}, 2 \mathrm{H}), 4.69(\mathrm{~s}, 1 \mathrm{H}) ;{ }^{13} \mathrm{C}$ NMR $(100$ $\left.\mathrm{MHz}, \mathrm{CDCl}_{3}\right) \delta 150.6,148.2,141.5,108.3,106.9,101.2,98.4$.<smiles>Oc1cccc2ccccc12</smiles>

2q

2q: pale yellow solid; ${ }^{1} \mathrm{H} \mathrm{NMR}\left(400 \mathrm{MHz}, \mathrm{CDCl}_{3}\right) \delta$ 8.20-8.14 (m, 1H), 7.84-7.77 (m, 1H), 7.52-7.45 (m, 2H), $7.44(\mathrm{~d}, J=8.3 \mathrm{~Hz}, 1 \mathrm{H}), 7.33-7.26(\mathrm{~m}, 1 \mathrm{H}), 6.80$ (dd, $J$ $=7.4,0.5 \mathrm{~Hz}, 1 \mathrm{H}), 5.32(\mathrm{~s}, 1 \mathrm{H}) ;{ }^{13} \mathrm{C} \mathrm{NMR}\left(100 \mathrm{MHz}, \mathrm{CDCl}_{3}\right) \delta 151.5,134.8,127.7$, $126.5,125.9,125.3,124.4,121.6,120.7,108.7$.

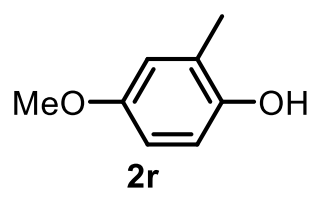

2r: white solid; ${ }^{1} \mathrm{H}$ NMR (400 MHz, $\left.\mathrm{CDCl}_{3}\right) \delta$ 6.77-6.67 (m, 2H), $6.63(\mathrm{dd}, J=8.7$, $2.9 \mathrm{~Hz}, 1 \mathrm{H}), 4.87(\mathrm{~s}, 1 \mathrm{H}), 3.76(\mathrm{~s}, 3 \mathrm{H}), 2.24(\mathrm{~s}, 3 \mathrm{H}) ;{ }^{13} \mathrm{C} \mathrm{NMR}\left(100 \mathrm{MHz}, \mathrm{CDCl}_{3}\right) \delta$ $153.6,148.0,125.2,116.8,115.7,112.0,55.9,16.2$.

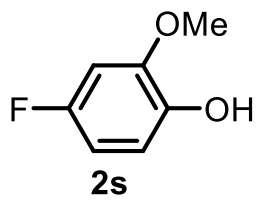


2s: pale yellow oil; ${ }^{1} \mathrm{H}$ NMR $\left(400 \mathrm{MHz}, \mathrm{CDCl}_{3}\right) \delta 6.75(\mathrm{dd}, J=8.9,5.1 \mathrm{~Hz}, 1 \mathrm{H})$, $6.71(\mathrm{dd}, J=9.5,3.0 \mathrm{~Hz}, 1 \mathrm{H}), 6.54(\mathrm{td}, J=8.7,3.0 \mathrm{~Hz}, 1 \mathrm{H}), 6.02(\mathrm{~s}, 1 \mathrm{H}), 3.83(\mathrm{~s}$, $3 \mathrm{H}) ;{ }^{13} \mathrm{C} \mathrm{NMR}\left(100 \mathrm{MHz}, \mathrm{CDCl}_{3}\right) \delta 157.6(\mathrm{~d}, J=237.8 \mathrm{~Hz}), 146.5(\mathrm{~d}, J=12.6 \mathrm{~Hz})$, $143.1(\mathrm{~d}, J=2.6 \mathrm{~Hz}), 111.1(\mathrm{~d}, J=10.0 \mathrm{~Hz}), 105.6(\mathrm{~d}, J=23.0 \mathrm{~Hz}), 102.8(\mathrm{~d}, J=$ $27.0 \mathrm{~Hz}), 56.4$.

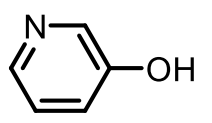

2t

2t: white brown solid; ${ }^{1} \mathrm{H}$ NMR (400 MHz, DMSO- $\left.d_{6}\right) \delta 9.89$ (br s, $\left.1 \mathrm{H}\right), 8.15$ (s, $1 \mathrm{H}$ ), $8.03(\mathrm{~d}, J=3.5 \mathrm{~Hz}, 1 \mathrm{H}), 7.23-7.11(\mathrm{~m}, 1 \mathrm{H}) ;{ }^{13} \mathrm{C}$ NMR $\left(100 \mathrm{MHz}, \mathrm{DMSO}-d_{6}\right) \delta$ $153.8,140.2,138.0,124.2,122.1$.<smiles>Cc1ccc2cccc(O)c2n1</smiles>

2u: light brown solid; ${ }^{1} \mathrm{H}$ NMR (400 MHz, DMSO- $d_{6}$ ) $\delta 9.41$ (br s, $\left.1 \mathrm{H}\right), 8.15$ (d, $J=$ 8.4 Hz, 1H), 7.45-7.24 (m, 3H), $7.07(\mathrm{~d}, J=6.6 \mathrm{~Hz}, 1 \mathrm{H}), 2.67(\mathrm{~s}, 3 \mathrm{H}) ;{ }^{13} \mathrm{C} \mathrm{NMR}$ $\left(100 \mathrm{MHz}, \mathrm{DMSO}-d_{6}\right) \delta 156.6,152.5,137.8,136.1,126.9,126.4,122.6,117.5$, 111.1, 24.7.<smiles>Cc1c(O)ccc2cccnc12</smiles>

2v: brownish grey solid; ${ }^{1} \mathrm{H}$ NMR (400 MHz, DMSO-d $\left.d_{6}\right) \delta 9.84(\mathrm{~s}, 1 \mathrm{H}), 8.80$ (dd, $J$ $=4.1,1.6 \mathrm{~Hz}, 1 \mathrm{H}), 8.15(\mathrm{dd}, J=8.1,1.5 \mathrm{~Hz}, 1 \mathrm{H}), 7.64(\mathrm{~d}, J=8.8 \mathrm{~Hz}, 1 \mathrm{H}), 7.31-7.21$ $(\mathrm{m}, 2 \mathrm{H}), 2.54(\mathrm{~s}, 3 \mathrm{H}) ;{ }^{13} \mathrm{C}$ NMR (100 MHz, DMSO- $\left.d_{6}\right) \delta 155.4,149.5,148.1,135.9$, $126.0,122.4,118.5,117.9,117.4,9.7$.<smiles>Cc1ccc2ccc(O)cc2n1</smiles>

2w: white solid; ${ }^{1} \mathrm{H}$ NMR (400 MHz, DMSO- $\left.d_{6}\right) \delta 10.08(\mathrm{~s}, 1 \mathrm{H}), 8.05(\mathrm{~d}, J=8.3 \mathrm{~Hz}$, 1H), $7.72(\mathrm{~d}, J=8.8 \mathrm{~Hz}, 1 \mathrm{H}), 7.19-7.12(\mathrm{~m}, 2 \mathrm{H}), 7.08(\mathrm{dd}, J=8.8,2.4 \mathrm{~Hz}, 1 \mathrm{H}), 2.57$ 
$(\mathrm{s}, 3 \mathrm{H}) ;{ }^{13} \mathrm{C}$ NMR (100 MHz, DMSO-d $\left.d_{6}\right) \delta 158.6,158.5,149.2,135.7,128.9,120.4$, 119.0, 118.1, 109.6, 24.8.

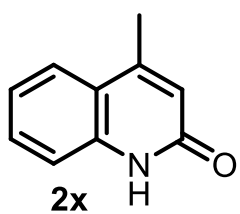

2x: grey solid; ${ }^{1} \mathrm{H}$ NMR (400 MHz, DMSO- $\left.d_{6}\right) \delta 11.60(\mathrm{~s}, 1 \mathrm{H}), 7.67(\mathrm{~d}, J=7.9 \mathrm{~Hz}$, 1H), $7.48(\mathrm{t}, J=7.5 \mathrm{~Hz}, 1 \mathrm{H}), 7.31(\mathrm{~d}, J=8.1 \mathrm{~Hz}, 1 \mathrm{H}), 7.17(\mathrm{t}, J=7.5 \mathrm{~Hz}, 1 \mathrm{H}), 6.39$ (s, 1H), 2.40 (s, 3H); ${ }^{13} \mathrm{C}$ NMR (100 MHz, DMSO-d $d_{6} \delta 161.6,147.9,138.7,130.2$, $124.7,121.6,120.8,119.6,115.4,18.4$.<smiles>Oc1ccc2ncccc2c1</smiles>

2y: pale yellow solid; ${ }^{1} \mathrm{H}$ NMR (400 MHz, DMSO- $\left.d_{6}\right) \delta 10.03(\mathrm{~s}, 1 \mathrm{H}), 8.65$ (dd, $J=$ 4.1, $1.5 \mathrm{~Hz}, 1 \mathrm{H}), 8.12(\mathrm{~d}, J=7.7 \mathrm{~Hz}, 1 \mathrm{H}), 7.87(\mathrm{~d}, J=9.1 \mathrm{~Hz}, 1 \mathrm{H}), 7.38(\mathrm{dd}, J=8.3$, $4.2 \mathrm{~Hz}, 1 \mathrm{H}), 7.32(\mathrm{dd}, J=9.1,2.7 \mathrm{~Hz}, 1 \mathrm{H}), 7.15(\mathrm{~d}, J=2.6 \mathrm{~Hz}, 1 \mathrm{H}) ;{ }^{13} \mathrm{C}$ NMR $(100$ MHz, DMSO- $\left.d_{6}\right) \delta 155.5,147.1,143.0,134.1,130.4,129.3,122.0,121.4,108.4$.<smiles>Oc1cncc2ccccc12</smiles>

2z: brown solid); ${ }^{1} \mathrm{H}$ NMR (400 MHz, DMSO-d $\left.d_{6}\right) \delta 10.41$ (s, 1H), 8.80 (s, 1H), 8.13 $(\mathrm{d}, J=8.4 \mathrm{~Hz}, 1 \mathrm{H}), 8.09(\mathrm{~s}, 1 \mathrm{H}), 8.02(\mathrm{~d}, J=8.1 \mathrm{~Hz}, 1 \mathrm{H}), 7.71(\mathrm{ddd}, J=8.3,6.9,1.2$ $\mathrm{Hz}, 1 \mathrm{H}), 7.67-7.60(\mathrm{~m}, 1 \mathrm{H}) ;{ }^{13} \mathrm{C}$ NMR $\left(100 \mathrm{MHz}, \mathrm{DMSO}-d_{6}+\mathrm{D}_{2} \mathrm{O}\right) \delta 148.8,143.3$, $129.7,129.5,128.0,127.35,127.3,126.9,121.3$.<smiles>Oc1cc[12cH+]c2nccnc2c1</smiles>

2aa: yellow solid; ${ }^{1} \mathrm{H}$ NMR (400 MHz, DMSO- $\left.d_{6}\right) \delta 10.51(\mathrm{~s}, 1 \mathrm{H}), 8.77(\mathrm{~d}, J=1.8$ Hz, 1H), 8.69 (d, $J=1.8 \mathrm{~Hz}, 1 \mathrm{H}), 7.93(\mathrm{~d}, J=9.1 \mathrm{~Hz}, 1 \mathrm{H}), 7.41(\mathrm{dd}, J=9.1,2.7 \mathrm{~Hz}$, $1 \mathrm{H}), 7.27(\mathrm{~d}, J=2.7 \mathrm{~Hz}, 1 \mathrm{H}) ;{ }^{13} \mathrm{C}$ NMR $\left(100 \mathrm{MHz}, \mathrm{DMSO}-d_{6}\right) \delta 158.8,145.4,144.0$, 142.1, 137.6, 130.4, 122.9, 119.5. 


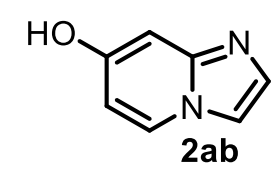

2ab: white brown solid; ${ }^{1} \mathrm{H}$ NMR (400 MHz, $\left.\mathrm{CD}_{3} \mathrm{OD}\right) \delta 8.31(\mathrm{~d}, J=7.4 \mathrm{~Hz}, 1 \mathrm{H})$, $7.69(\mathrm{~d}, J=1.8 \mathrm{~Hz}, 1 \mathrm{H}), 7.50(\mathrm{~d}, J=1.8 \mathrm{~Hz}, 1 \mathrm{H}), 6.75(\mathrm{dd}, J=7.4,1.9 \mathrm{~Hz}, 1 \mathrm{H})$, 6.71-6.65 (m, 1H); ${ }^{13} \mathrm{C}$ NMR (100 MHz, DMSO- $\left.d_{6}\right) \delta$ 159.1, 144.7, 128.6, 127.4, 112.3, 109.0, 94.9. ESI-HRMS: $m / z$ calcd for $\mathrm{C}_{7} \mathrm{H}_{6} \mathrm{~N}_{2} \mathrm{NaO}(\mathrm{M}+\mathrm{Na})^{+}$157.0378, found: 157.0371 .

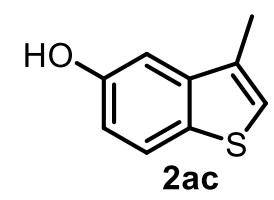

2ac: pale yellow solid; ${ }^{1} \mathrm{H} \mathrm{NMR}\left(400 \mathrm{MHz}, \mathrm{CDCl}_{3}\right) \delta{ }^{1} \mathrm{H} \mathrm{NMR}\left(400 \mathrm{MHz}, \mathrm{CDCl}_{3}\right) \delta$ $7.69(\mathrm{~d}, J=8.6 \mathrm{~Hz}, 1 \mathrm{H}), 7.14(\mathrm{~d}, J=2.4 \mathrm{~Hz}, 1 \mathrm{H}), 7.09$ (s, 1H), 6.94 (dd, $J=8.6,2.4$ $\mathrm{Hz}, 1 \mathrm{H}), 5.28(\mathrm{~s}, 1 \mathrm{H}), 2.36(\mathrm{~d}, J=1.0 \mathrm{~Hz}, 3 \mathrm{H}) ;{ }^{13} \mathrm{C} \mathrm{NMR}\left(100 \mathrm{MHz}, \mathrm{CDCl}_{3}\right) \delta 153.0$, 141.1, 132.8, 131.7, 123.7, 123.2, 114.1, 107.1, 14.0. ESI-HRMS: m/z calcd for $\mathrm{C}_{9} \mathrm{H}_{7} \mathrm{OS}(\mathrm{M}-\mathrm{H})^{-}$163.0218, found: 163.0225 .

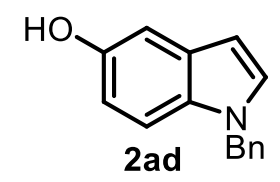

2ad: brown solid; ${ }^{1} \mathrm{H}$ NMR (400 MHz, DMSO- $\left.d_{6}\right) \delta 8.69$ (s, 1H), 7.37 (d, $J=3.0 \mathrm{~Hz}$, 1H), 7.32-7.15 (m, 6H), 6.89 (d, $J=2.1 \mathrm{~Hz}, 1 \mathrm{H}), 6.63(\mathrm{dd}, J=8.7,2.1 \mathrm{~Hz}, 1 \mathrm{H}), 6.29$ $(\mathrm{d}, J=2.9 \mathrm{~Hz}, 1 \mathrm{H}), 5.32(\mathrm{~s}, 2 \mathrm{H}) ;{ }^{13} \mathrm{C}$ NMR $\left(100 \mathrm{MHz}, \mathrm{DMSO}-d_{6}\right) \delta 150.9,138.5$, $130.4,129.3,129.1,128.4,127.2,126.9,111.4,110.4,104.3,100.0,49.2$.

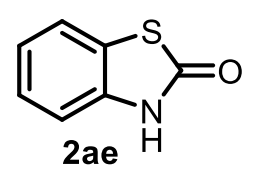

2ae: brown solid; ${ }^{1} \mathrm{H}$ NMR (400 MHz, $\mathrm{CDCl}_{3}$ ) $\delta 10.49$ (s, 1H), 7.39 (d, J= $7.5 \mathrm{~Hz}$, 1H), 7.31-7.07 (m, 3H); ${ }^{13} \mathrm{C}$ NMR (100 MHz, $\left.\mathrm{CDCl}_{3}\right) \delta$ 173.6, 135.7, 126.6, 124.0, 123.3,122.5, 112.1. 
<smiles>O=c1c2ccccc2sc2ccc(O)cc12</smiles>

2af: yellow solid; ${ }^{1} \mathrm{H}$ NMR (400 MHz, DMSO- $\left.d_{6}\right) \delta 10.14(\mathrm{~s}, 1 \mathrm{H}), 8.44(\mathrm{dd}, J=8.1$, $1.1 \mathrm{~Hz}, 1 \mathrm{H}), 7.86(\mathrm{~d}, J=2.8 \mathrm{~Hz}, 1 \mathrm{H}), 7.77(\mathrm{~d}, J=7.7 \mathrm{~Hz}, 1 \mathrm{H}), 7.75-7.69$ (m, 1H), $7.66(\mathrm{~d}, J=8.7 \mathrm{~Hz}, 1 \mathrm{H}), 7.57-7.50(\mathrm{~m}, 1 \mathrm{H}), 7.26(\mathrm{dd}, J=8.7,2.8 \mathrm{~Hz}, 1 \mathrm{H}) ;{ }^{13} \mathrm{C} \mathrm{NMR}$ $\left(100 \mathrm{MHz}, \mathrm{DMSO}-d_{6}\right) \delta 178.6,156.4,137.0,132.6,129.6,129.1,128.0,127.7$, $126.5,126.3,126.1,122.7,113.2$.

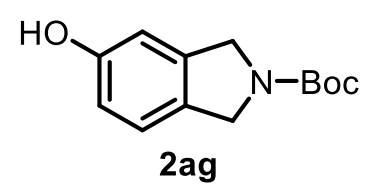

2ag: white solid; ${ }^{1} \mathrm{H}$ NMR (400 MHz, DMSO- $\left.d_{6}\right) \delta 9.37(\mathrm{~s}, 1 \mathrm{H}), 7.13-7.01(\mathrm{~m}, 1 \mathrm{H})$, 6.75-6.61 (m, 2H), $4.46(\mathrm{dd}, J=15.1,9.1 \mathrm{~Hz}, 4 \mathrm{H}), 3.38(\mathrm{~s}, 1 \mathrm{H}), 1.44(\mathrm{~s}, 9 \mathrm{H}) ;{ }^{13} \mathrm{C}$ NMR (100 MHz, DMSO-d $\left.d_{6}\right) \delta 156.9,153.6(\mathrm{~d}, J=2.8 \mathrm{~Hz}), 138.0(\mathrm{~d}, J=55.3 \mathrm{~Hz})$, $126.7(\mathrm{~d}, J=51.2 \mathrm{~Hz}), 123.4,114.7,109.3,78.7,51.8(\mathrm{~d}, J=15.1 \mathrm{~Hz}), 51.24(\mathrm{~d}, J=$ 16.6 Hz), 28.2; ESI-HRMS: $m / z$ calcd for $\mathrm{C}_{13} \mathrm{H}_{17} \mathrm{KO}_{3}(\mathrm{M}+\mathrm{K})^{+}$274.0846, found 274.0844 .<smiles>Oc1cnc2ccccc2c1</smiles>

2ah: yellow solid; ${ }^{1} \mathrm{H}$ NMR (400 MHz, DMSO- $\left.d_{6}\right) \delta 10.31(\mathrm{~s}, 1 \mathrm{H}), 8.62(\mathrm{~s}, 1 \mathrm{H})$, 8.04-7.68 (m, 2H), 7.61-7.34 (m, 3H); ${ }^{13} \mathrm{C}$ NMR (100 MHz, DMSO-d 6$) \delta 150.9$, $144.0,142.5,129.1,128.6,126.7,126.5,125.8,115.3$.

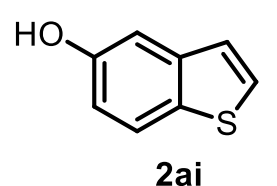

2ai: pink solid; ${ }^{1} \mathrm{H}$ NMR (400 MHz, $\left.\mathrm{CDCl}_{3}\right) \delta 7.72(\mathrm{~d}, J=8.7 \mathrm{~Hz}, 1 \mathrm{H}), 7.45$ (d, $J=$ $5.4 \mathrm{~Hz}, 1 \mathrm{H}), 7.25(\mathrm{~d}, J=2.4 \mathrm{~Hz}, 1 \mathrm{H}), 7.20(\mathrm{~d}, J=5.4 \mathrm{~Hz}, 1 \mathrm{H}), 6.94(\mathrm{dd}, J=8.7,2.4$ $\mathrm{Hz}, 1 \mathrm{H}), 5.32(\mathrm{~s}, 1 \mathrm{H}) ;{ }^{13} \mathrm{C} \mathrm{NMR}\left(100 \mathrm{MHz}, \mathrm{CDCl}_{3}\right) \delta 153.0,140.9,132.5,128.0$, $123.44,123.38,114.4,108.7$. 
<smiles>Oc1ccc2c(c1)c1ccccc1n2Cc1ccccc1</smiles>

2aj: white solid; ${ }^{1} \mathrm{H}$ NMR (400 MHz, $\left.\mathrm{CDCl}_{3}\right) \delta 7.99(\mathrm{~d}, J=7.8 \mathrm{~Hz}, 1 \mathrm{H}), 7.51(\mathrm{~d}, J=$ $2.4 \mathrm{~Hz}, 1 \mathrm{H}), 7.42-7.34(\mathrm{~m}, 1 \mathrm{H}), 7.29(\mathrm{~d}, J=8.2 \mathrm{~Hz}, 1 \mathrm{H}), 7.23-7.12(\mathrm{~m}, 5 \mathrm{H}), 7.08(\mathrm{dd}$, $J=5.5,2.3 \mathrm{~Hz}, 2 \mathrm{H}), 6.94(\mathrm{dd}, J=8.7,2.4 \mathrm{~Hz}, 1 \mathrm{H}), 5.40(\mathrm{~s}, 2 \mathrm{H}), 5.06(\mathrm{~s}, 1 \mathrm{H}) ;{ }^{13} \mathrm{C}$ NMR $\left(100 \mathrm{MHz}, \mathrm{CDCl}_{3}\right) \delta 149.3,141.5,137.4,135.9,128.9,127.5,126.5,126.1$, 123.8, 122.7, 120.6, 118.9, 114.9, 109.7, 109.1, 106.1, 46.7; ESI-HRMS: m/z calcd for $\mathrm{C}_{19} \mathrm{H}_{14} \mathrm{NO}(\mathrm{M}-\mathrm{H})^{-} 272.1075$, found 272.1080.

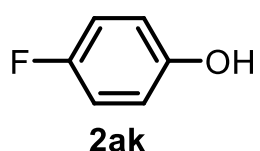

2ak: pale yellow solid; ${ }^{1} \mathrm{H}$ NMR $\left(400 \mathrm{MHz}, \mathrm{CDCl}_{3}\right) \delta$ 6.95-6.86 (m, 2H), 6.81-6.72 $(\mathrm{m}, 2 \mathrm{H}), 5.90(\mathrm{~s}, 1 \mathrm{H}) ;{ }^{13} \mathrm{C} \mathrm{NMR}\left(100 \mathrm{MHz}, \mathrm{CDCl}_{3}\right) \delta 157.4(\mathrm{~d}, J=237.8 \mathrm{~Hz}), 151.3$ $(\mathrm{d}, J=2.2 \mathrm{~Hz}), 116.4(\mathrm{~d}, J=8.1 \mathrm{~Hz}), 116.1(\mathrm{~d}, J=23.3 \mathrm{~Hz})$.

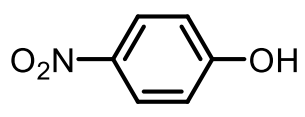

2al

2al: yellow solid; ${ }^{1} \mathrm{H}$ NMR $\left(400 \mathrm{MHz}, \mathrm{CDCl}_{3}\right) \delta 8.18(\mathrm{~d}, J=9.0 \mathrm{~Hz}, 2 \mathrm{H}), 6.94(\mathrm{~d}, J$ $=9.0 \mathrm{~Hz}, 2 \mathrm{H}), 6.21(\mathrm{~s}, 1 \mathrm{H}) ;{ }^{13} \mathrm{C} \mathrm{NMR}\left(100 \mathrm{MHz}, \mathrm{CDCl}_{3}\right) \delta 161.6,141.7,126.5$, 115.9.

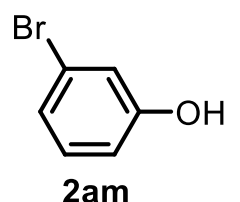

2am: light yellow solid; ${ }^{1} \mathrm{H}$ NMR (400 MHz, DMSO-d $\left.d_{6}\right) \delta 9.86(\mathrm{~s}, 1 \mathrm{H}), 7.12(\mathrm{t}, J=$ $8.3 \mathrm{~Hz}, 1 \mathrm{H}), 6.97-6.92(\mathrm{~m}, 2 \mathrm{H}), 6.77$ (ddd, $J=8.2,2.2,0.9 \mathrm{~Hz}, 1 \mathrm{H}) ;{ }^{13} \mathrm{C}$ NMR $(100$ MHz, DMSO- $\left.d_{6}\right) \delta 158.6,131.1,121.8,121.6,118.1,114.6$.

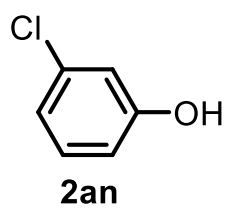


2an: white solid; ${ }^{1} \mathrm{H}$ NMR $\left(400 \mathrm{MHz}, \mathrm{CDCl}_{3}\right) \delta 7.16(\mathrm{t}, J=8.1 \mathrm{~Hz}, 1 \mathrm{H}), 6.92(\mathrm{ddd}, J$ $=8.0,1.9,0.8 \mathrm{~Hz}, 1 \mathrm{H}), 6.86(\mathrm{t}, J=2.2 \mathrm{~Hz}, 1 \mathrm{H}), 6.72(\mathrm{ddd}, J=8.2,2.4,0.8 \mathrm{~Hz}, 1 \mathrm{H})$, $4.96(\mathrm{~s}, 1 \mathrm{H}) ;{ }^{13} \mathrm{C} \mathrm{NMR}\left(100 \mathrm{MHz}, \mathrm{CDCl}_{3}\right) \delta$ 156.0, 135.0, 130.7, 121.4, 116.0, 113.9.<smiles>O=C(Nc1ccccc1)c1c(O)ccc[n+]1[O+]</smiles>

2ao: light yellow solid; ${ }^{1} \mathrm{H}$ NMR (400 MHz, $\left.\mathrm{CDCl}_{3}\right) \delta 11.94(\mathrm{~s}, 1 \mathrm{H}), 9.93(\mathrm{~s}, 1 \mathrm{H})$, $8.13(\mathrm{dd}, J=4.2,1.5 \mathrm{~Hz}, 1 \mathrm{H}), 7.79-7.68(\mathrm{~m}, 2 \mathrm{H}), 7.48-7.33(\mathrm{~m}, 4 \mathrm{H}), 7.19(\mathrm{t}, J=7.4$ $\mathrm{Hz}, 1 \mathrm{H}) ;{ }^{13} \mathrm{C} \mathrm{NMR}\left(100 \mathrm{MHz}, \mathrm{CDCl}_{3}\right) \delta 166.9,158.4,139.6,136.8,131.4,129.3$, $129.1,126.6,125.0,120.2$.<smiles>Oc1c[nH+]c2[nH]ccc2c1</smiles>

2ap: white solid; ${ }^{1} \mathrm{H}$ NMR (400 MHz, DMSO- $\left.d_{6}\right) \delta 9.17(\mathrm{~s}, 1 \mathrm{H}), 7.91(\mathrm{~d}, J=2.6 \mathrm{~Hz}$, 1H), $7.51(\mathrm{~d}, J=3.4 \mathrm{~Hz}, 1 \mathrm{H}), 7.33(\mathrm{~d}, J=2.6 \mathrm{~Hz}, 1 \mathrm{H}), 7.32-7.18(\mathrm{~m}, 5 \mathrm{H}), 6.34$ (d, $J$ $=3.4 \mathrm{~Hz}, 1 \mathrm{H}), 5.41(\mathrm{~s}, 2 \mathrm{H}) ;{ }^{13} \mathrm{C} \mathrm{NMR}\left(400 \mathrm{MHz}, \mathrm{DMSO}-d_{6}\right) \delta 148.4,142.2,138.7$, 132.6, 129.5, 128.4, 127.2, 127.1, 120.3, 113.1, 98.5, 47.2. ESI-HRMS: $\mathrm{m} / z$ calcd for $\mathrm{C}_{14} \mathrm{H}_{13} \mathrm{~N}_{2} \mathrm{O}(\mathrm{M}+\mathrm{H})^{+} 225.1028$, found: 225.1021 .

\section{References:}

1. Fan, M.; Zhou, W.; Jiang, Y.; Ma, D. Angew. Chem., Int. Ed. 2016, 48, 62116215.

2. Zhou, W.; Fan, M.; Yin, J.; Jiang, Y.; Ma, D. J. Am. Chem. Soc. 2015, 137,11942-11946. 


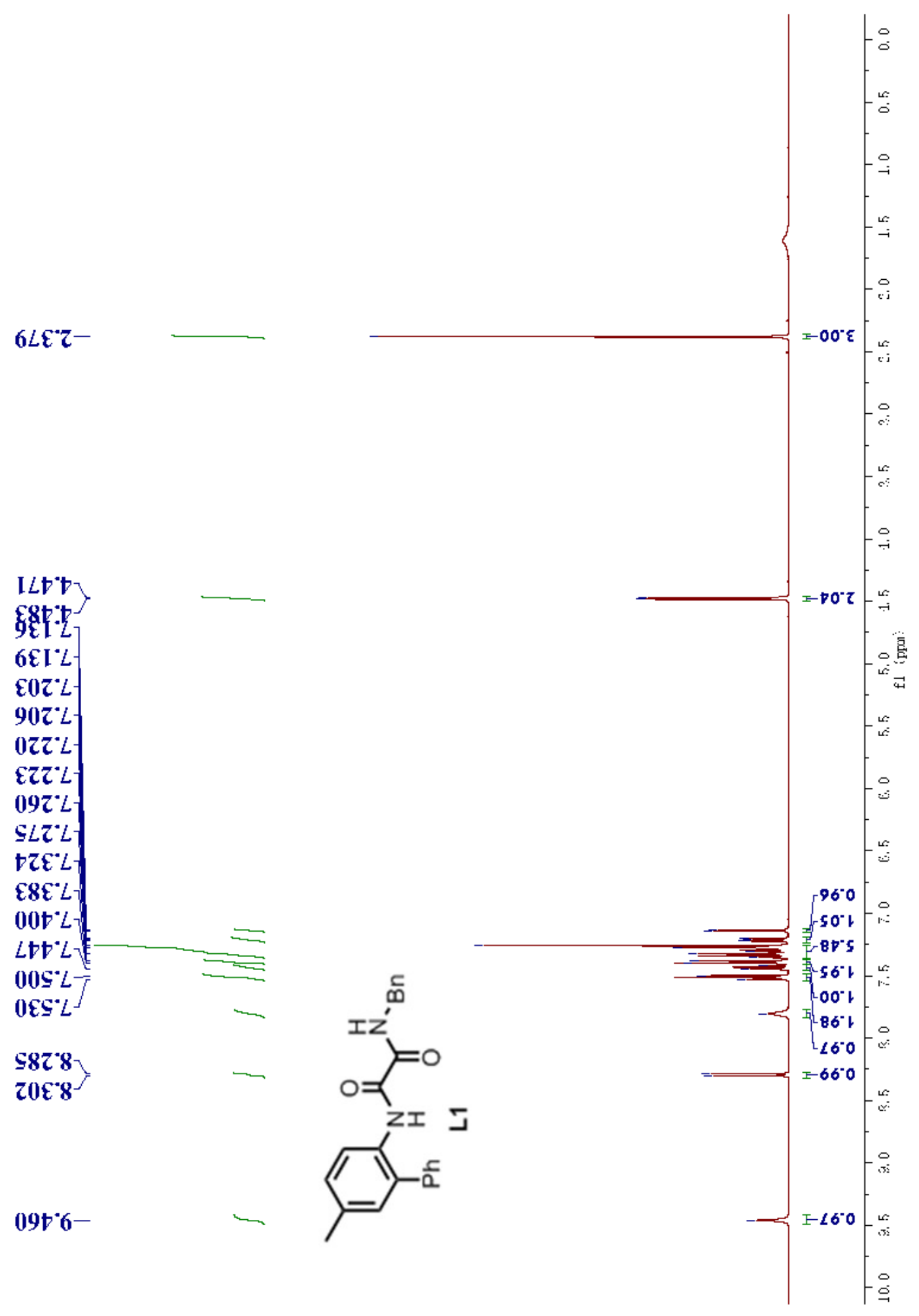

3. 


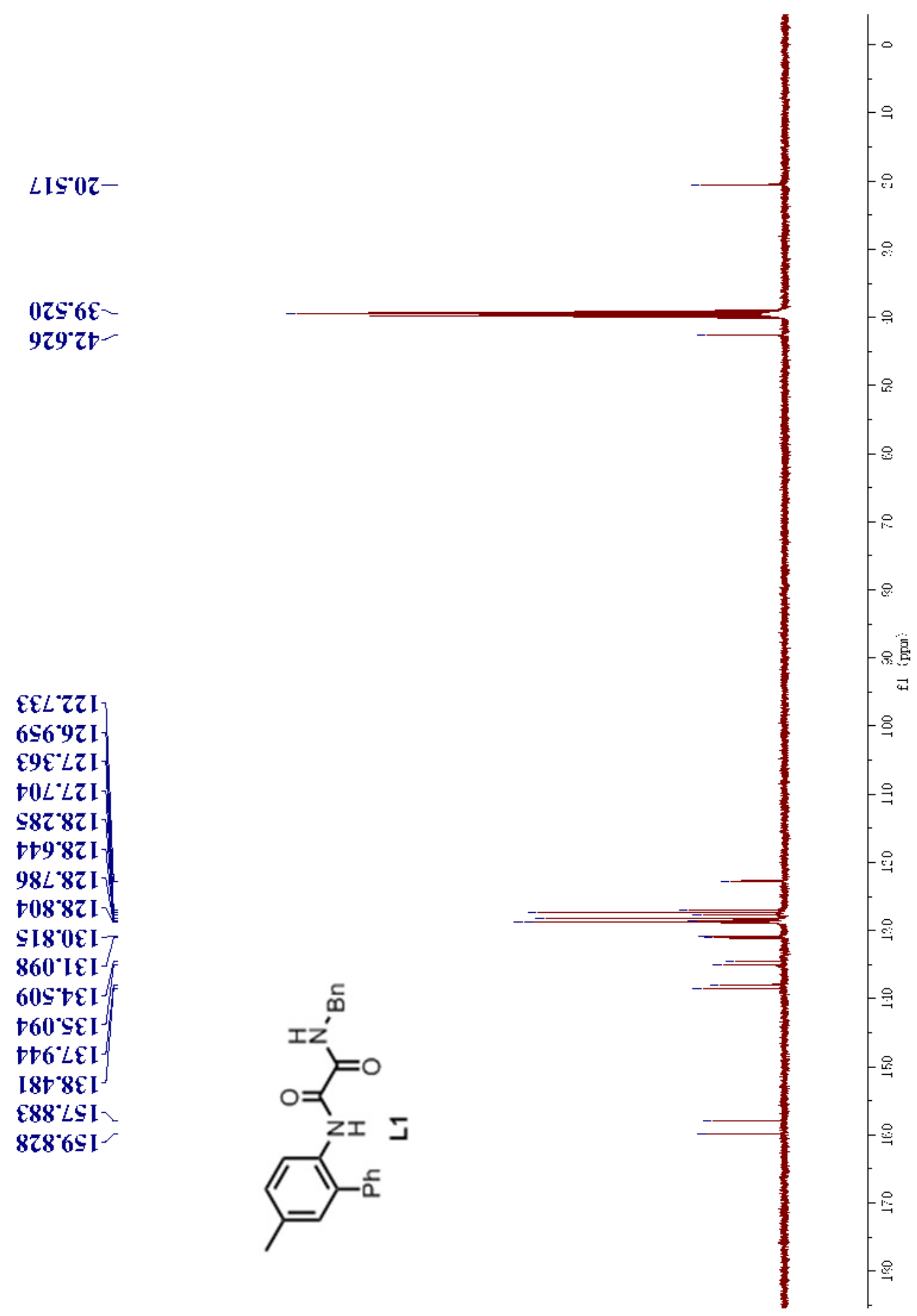




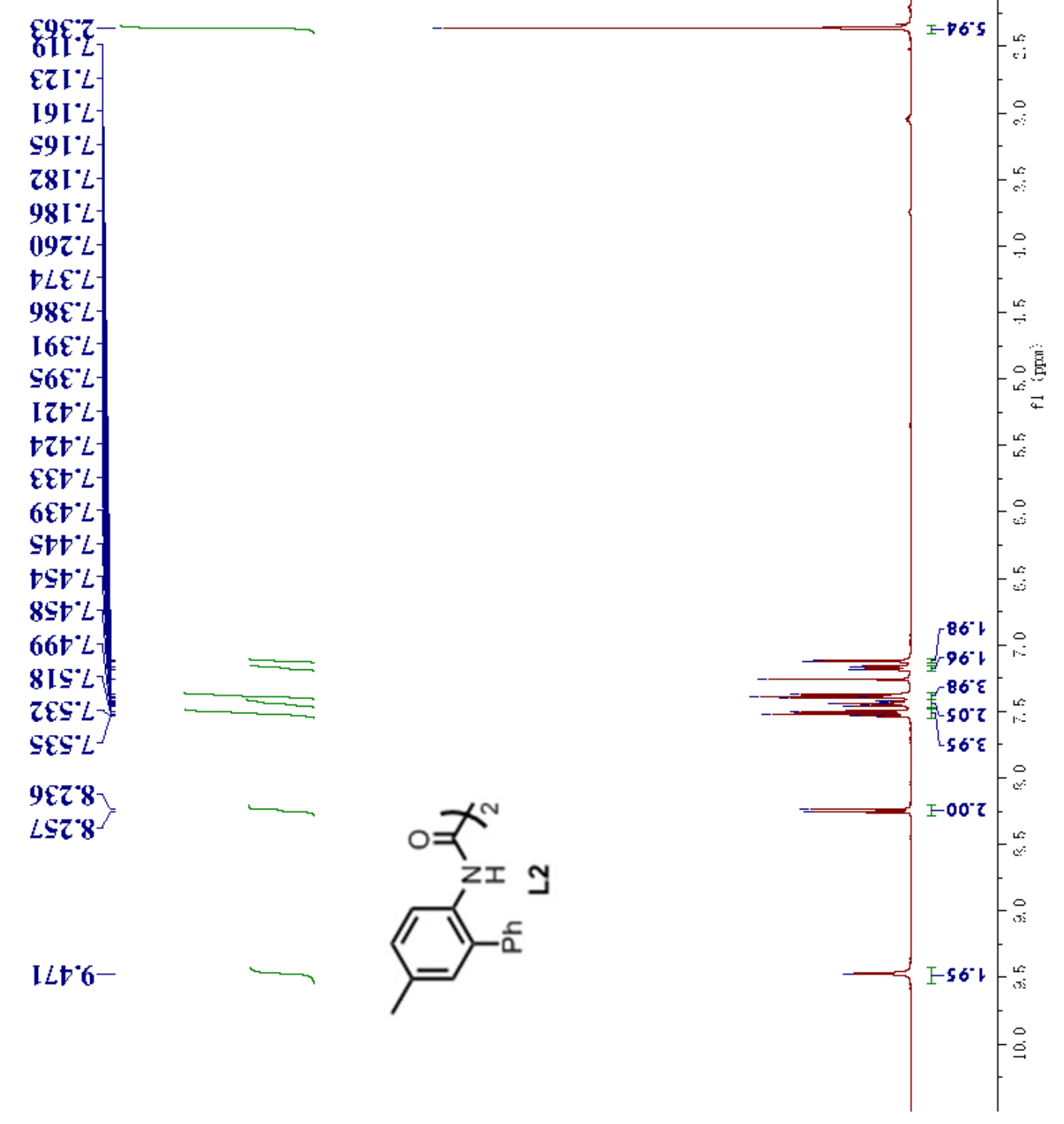


$\angle 80^{\circ} \mathrm{Iz}-$

$\left.\begin{array}{l}258^{\circ} 9 L \\ 091^{\circ} L L \\ L L 5^{\circ} L L\end{array}\right]$

$9 \varepsilon t^{\circ} 0 Z \mathrm{I}$

$\varepsilon$ It 82 I

$\$ 90 \% 2 \mathrm{I}$.

I8Z* $62 \mathrm{I}$

$6+\varepsilon * 6 z \mathrm{I}$

$96^{\circ} 0 \varepsilon \mathrm{I}-$

$\mathcal{E} \mathrm{II}^{\circ} \mathrm{IEI}$

$\varepsilon \angle 6^{\circ} Z \mathcal{E} I$

$\mathcal{E} I^{\prime} \subseteq \mathcal{E}{ }^{-}$

$9 S^{\prime} L E I^{\prime}$

$L E t^{*} L S I-$

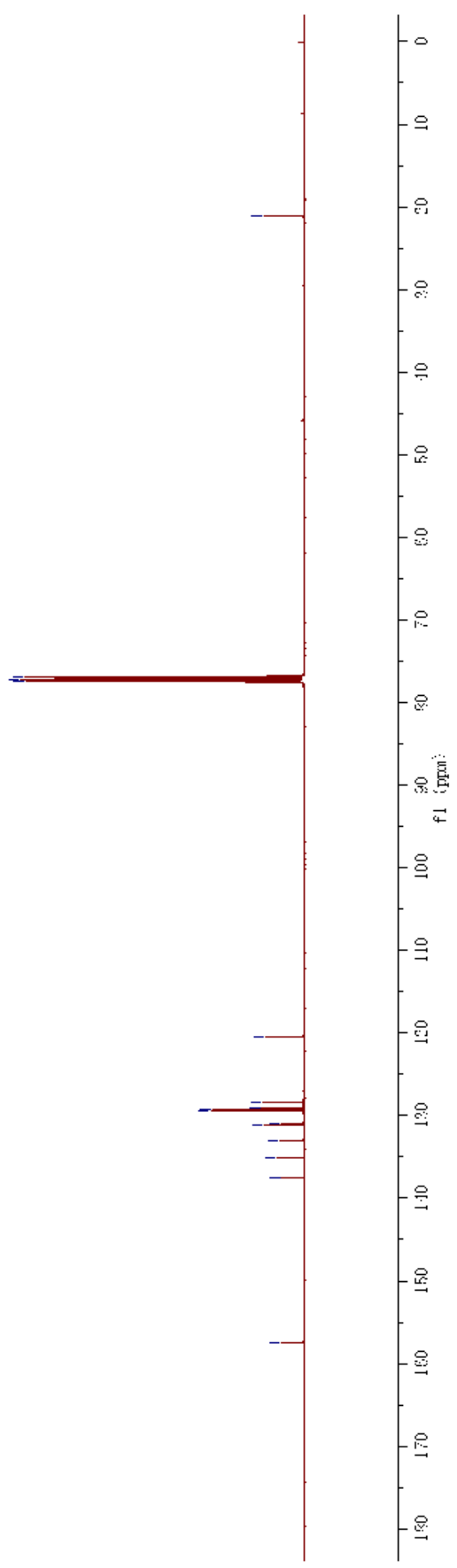



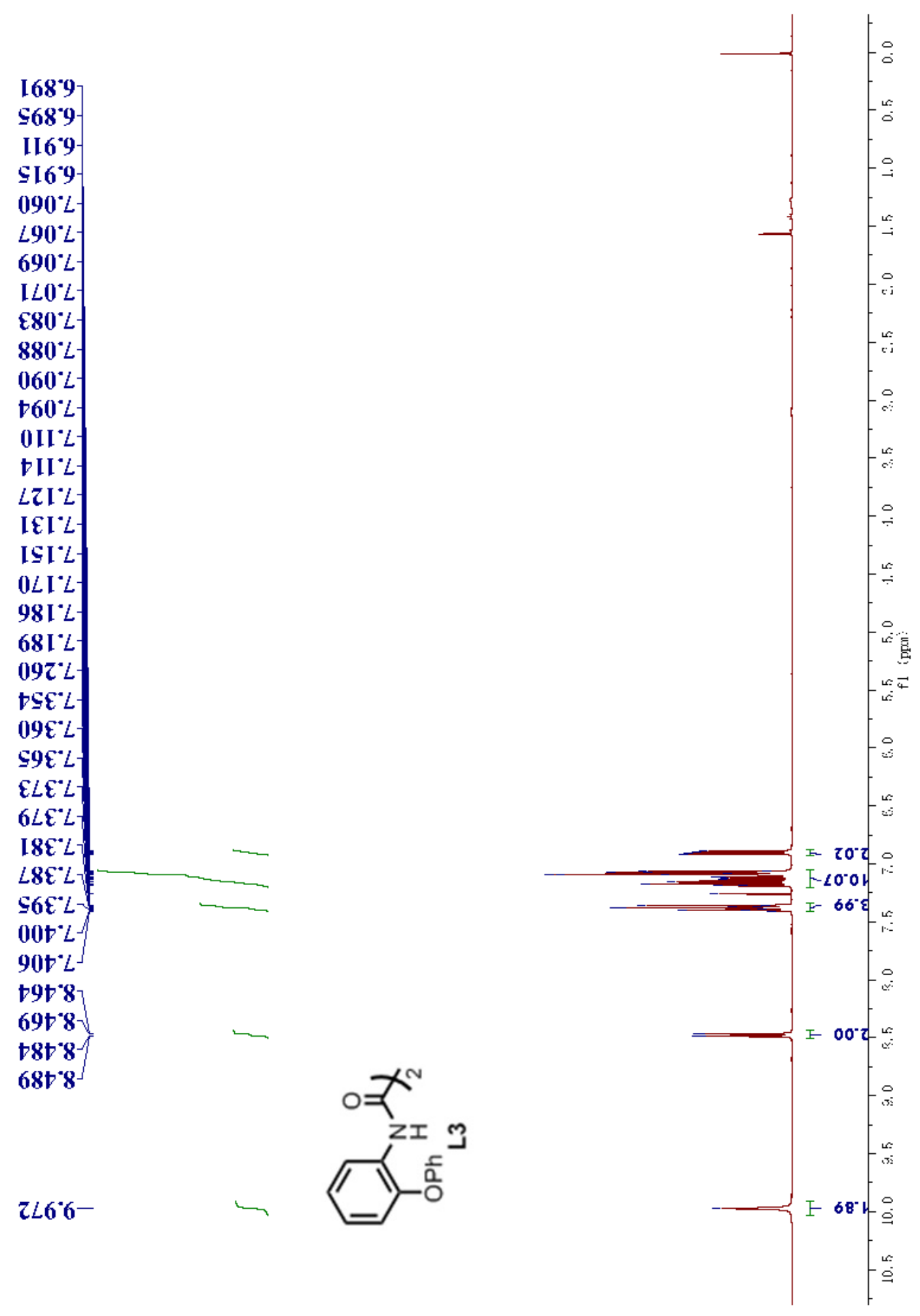


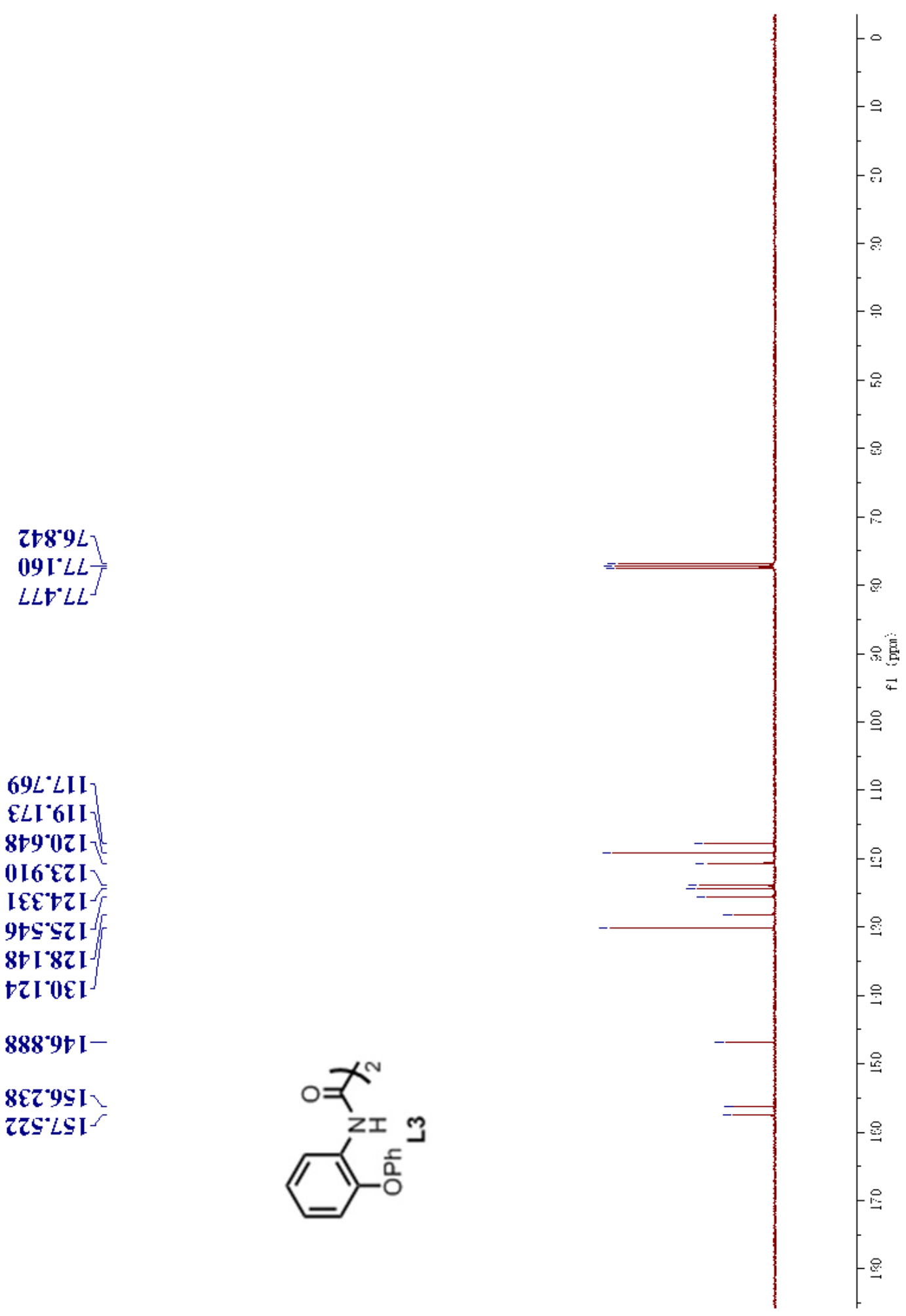




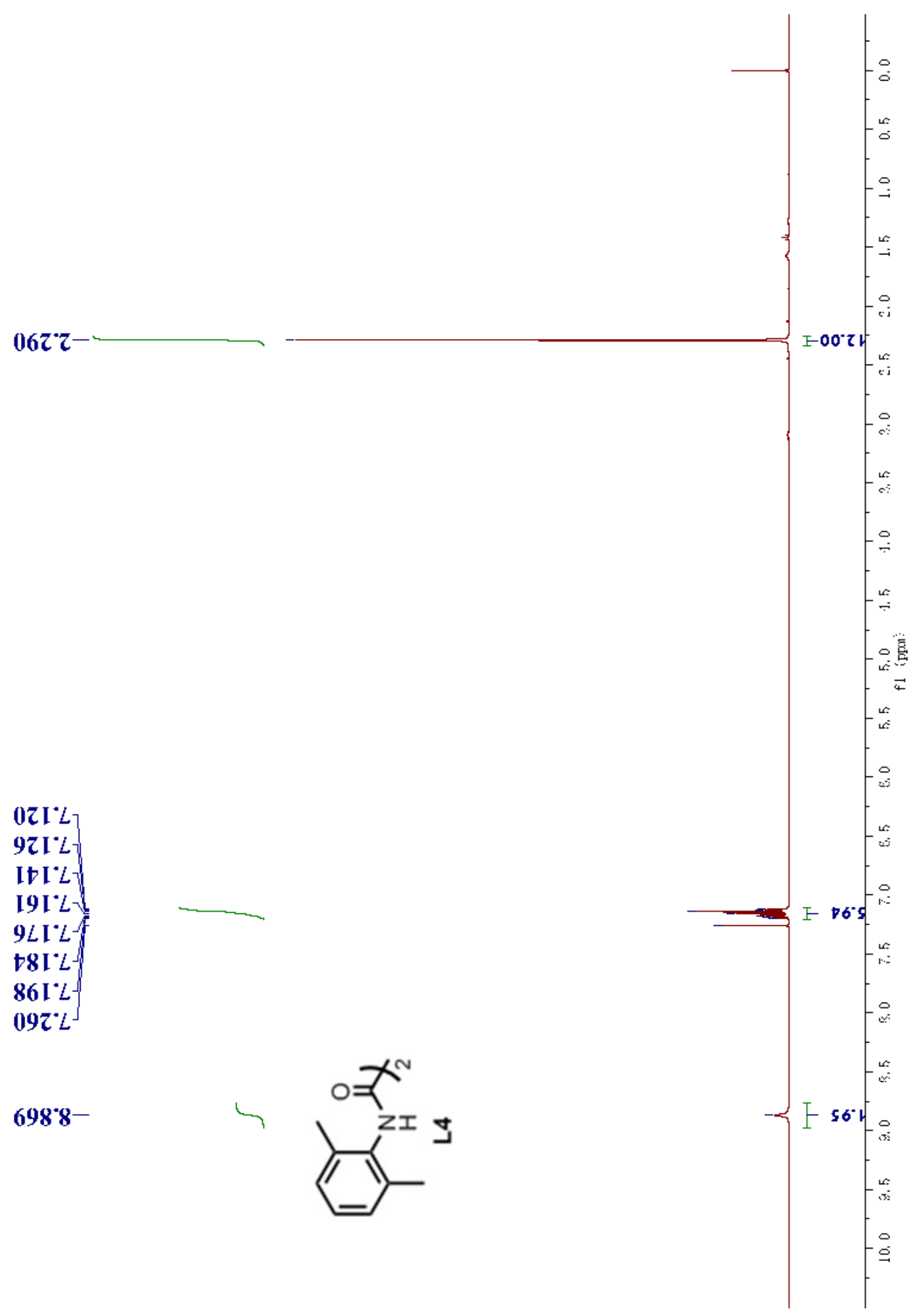




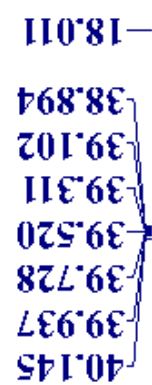

$000^{\circ} \angle Z$ I

$\angle 8 L^{\circ} L Z \mathrm{I}^{\circ}$

$Z \angle Z{ }^{\circ}+\varepsilon I$

$\tau \mathcal{Z} \mathbf{I}^{\circ} \subseteq \mathbf{I}^{\top}$

\$88.8SI-

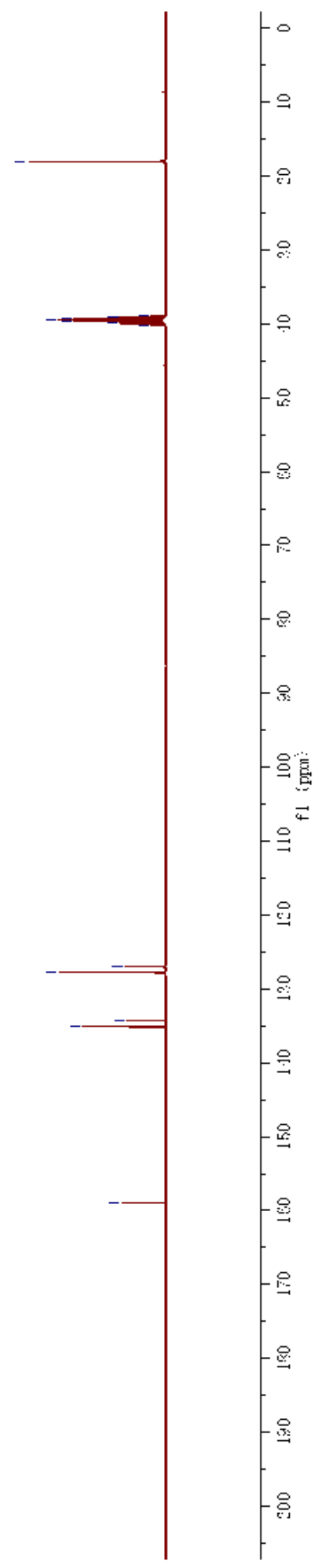




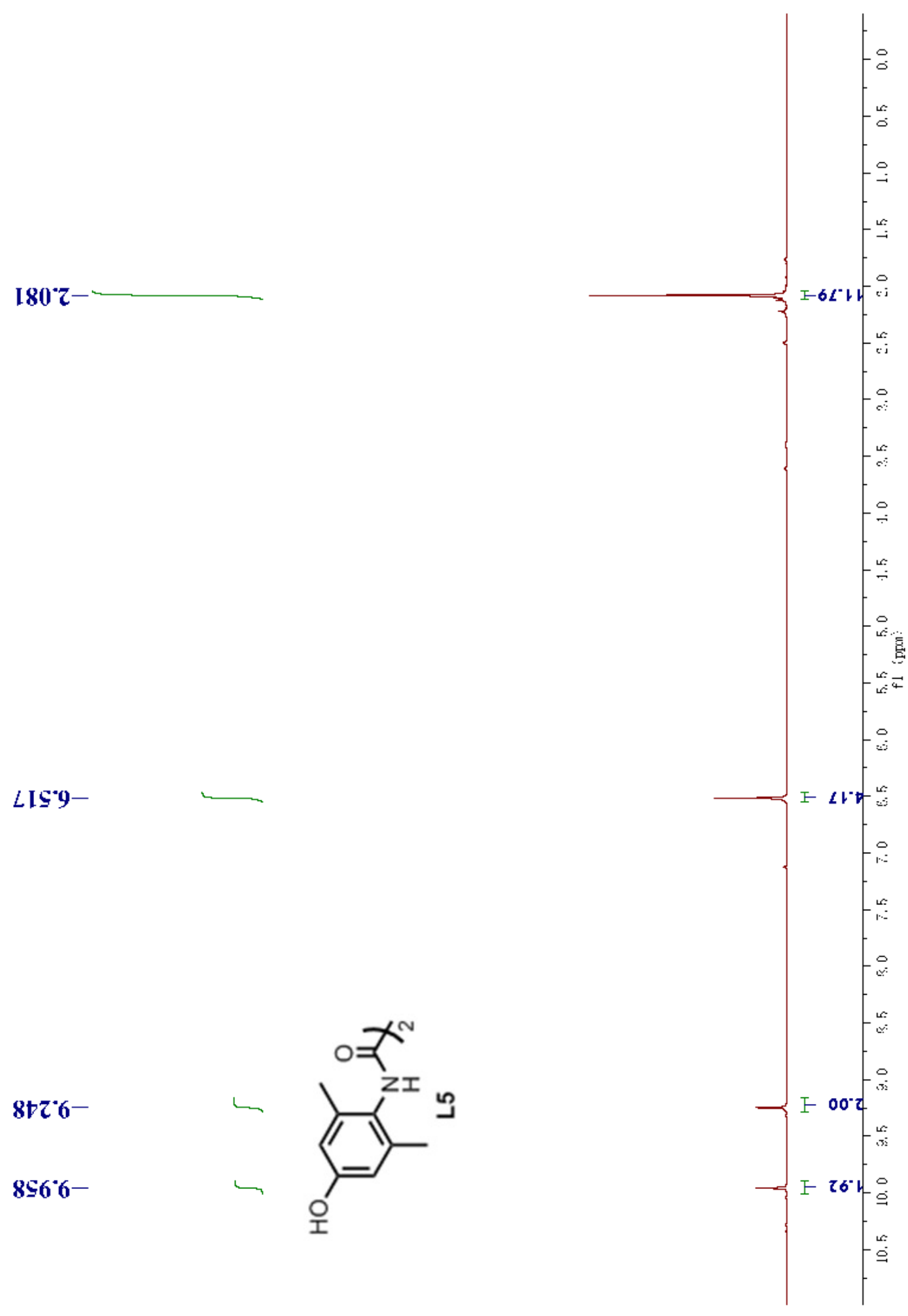


59I'8I-

$\$ 68.8 \varepsilon_{7}$

Z0I'6E

II $\varepsilon^{\prime \prime} 6 \varepsilon$

$0 z \subseteq 6$

$6 z L{ }^{\circ} 6 \varepsilon$

$\angle E 6^{\circ} 6 \varepsilon$

$9 \mathrm{II}^{\circ} 0 \mathrm{t}$

เE์เทI-

$69 \Im \Im Z I-$

$0 \mathcal{0} I^{\circ} 9 \mathcal{E} I-$

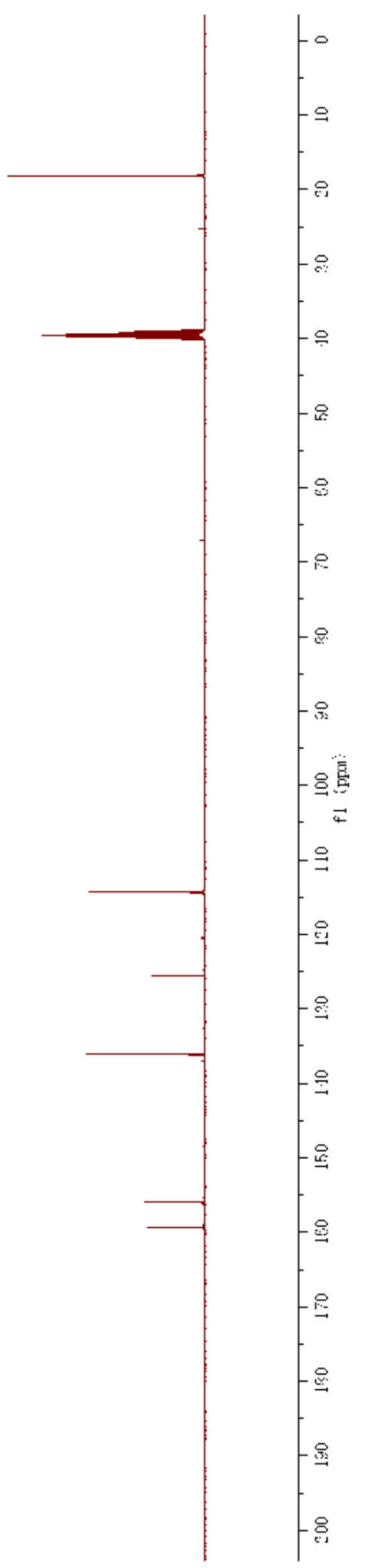

$\varepsilon 26^{\circ} \mathrm{SI}-$

$\angle E E^{*} 6 \mathrm{SI}^{-}$

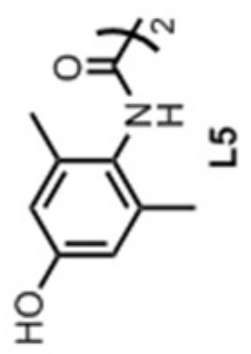


$6+z \cdot z$

$56 L^{\circ} \mathcal{E}-$

999\%-

$09 z^{\circ} L-$

Et $L 8-$

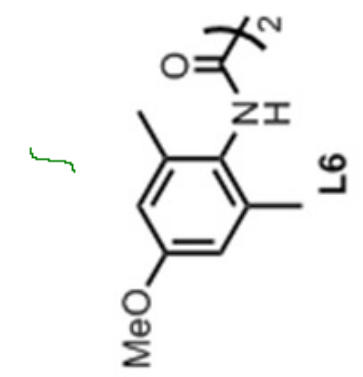

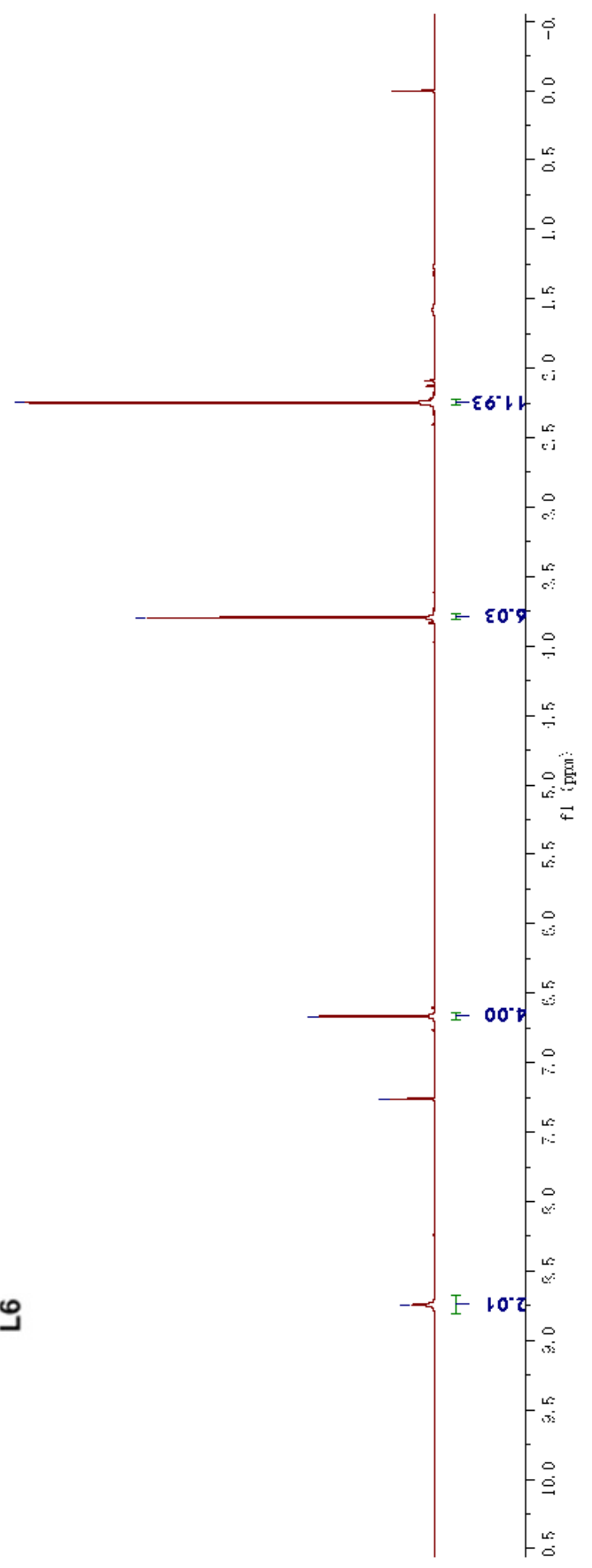




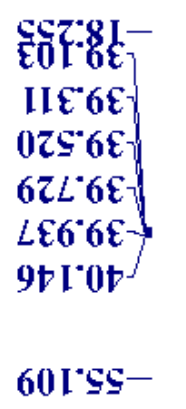

$906{ }^{\circ} \mathrm{LI}-$

$\$ 80^{\circ} L Z I-$

$\operatorname{StE}^{*} 9 \varepsilon \mathrm{I}-$

$89 L^{\circ} L S I$
$8+z^{\circ} 6 \mathrm{SI}^{\prime}$

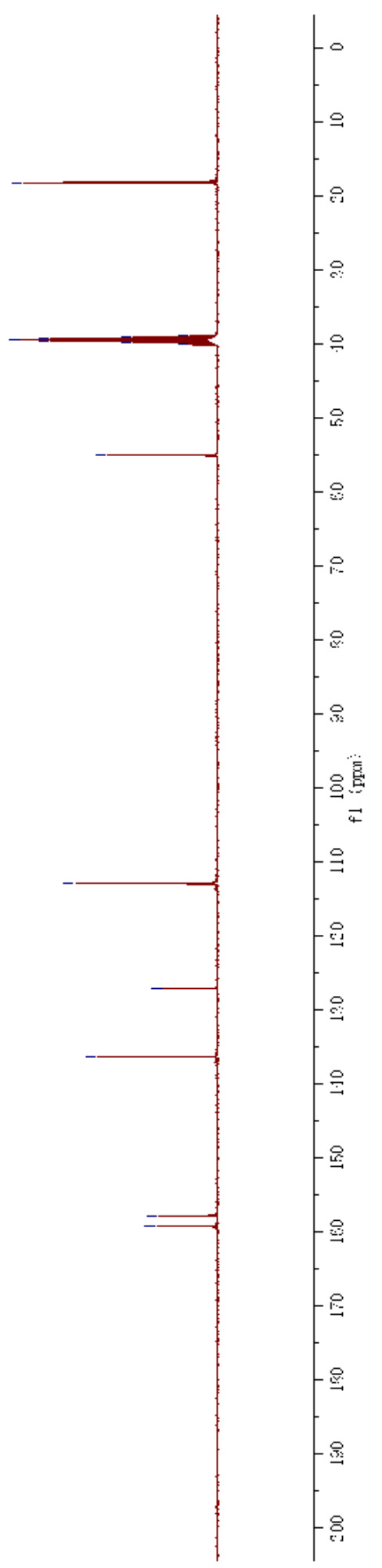




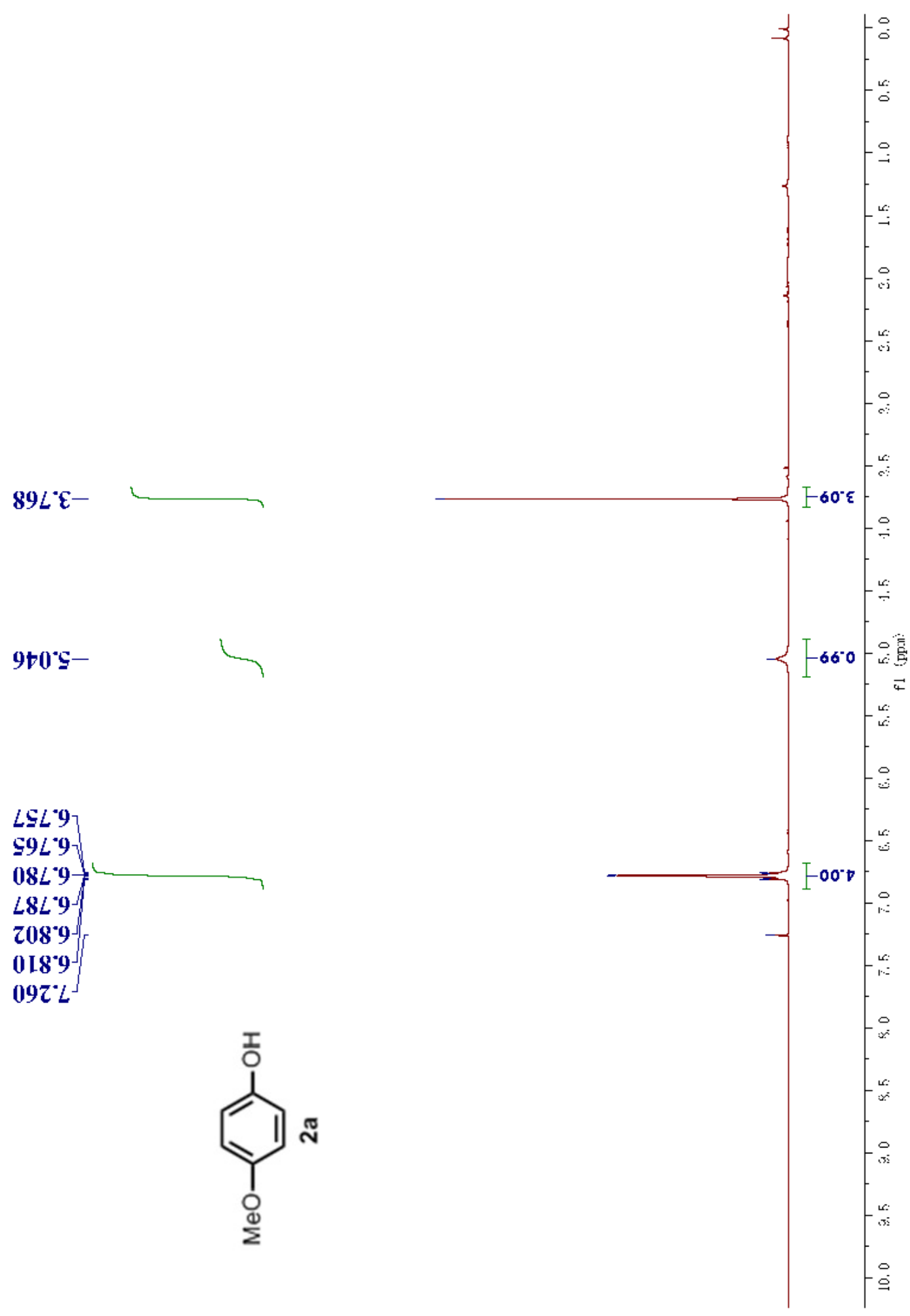




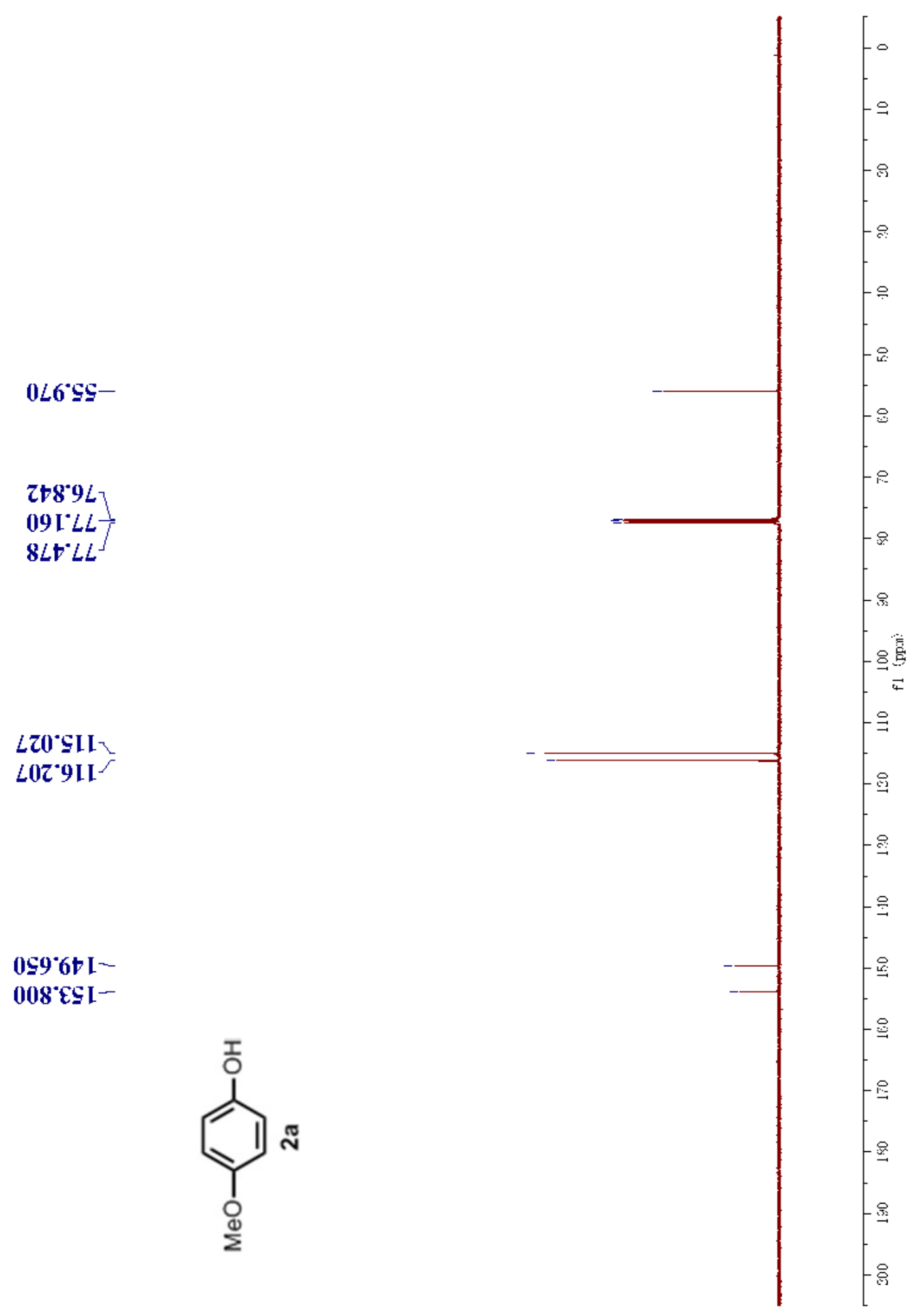




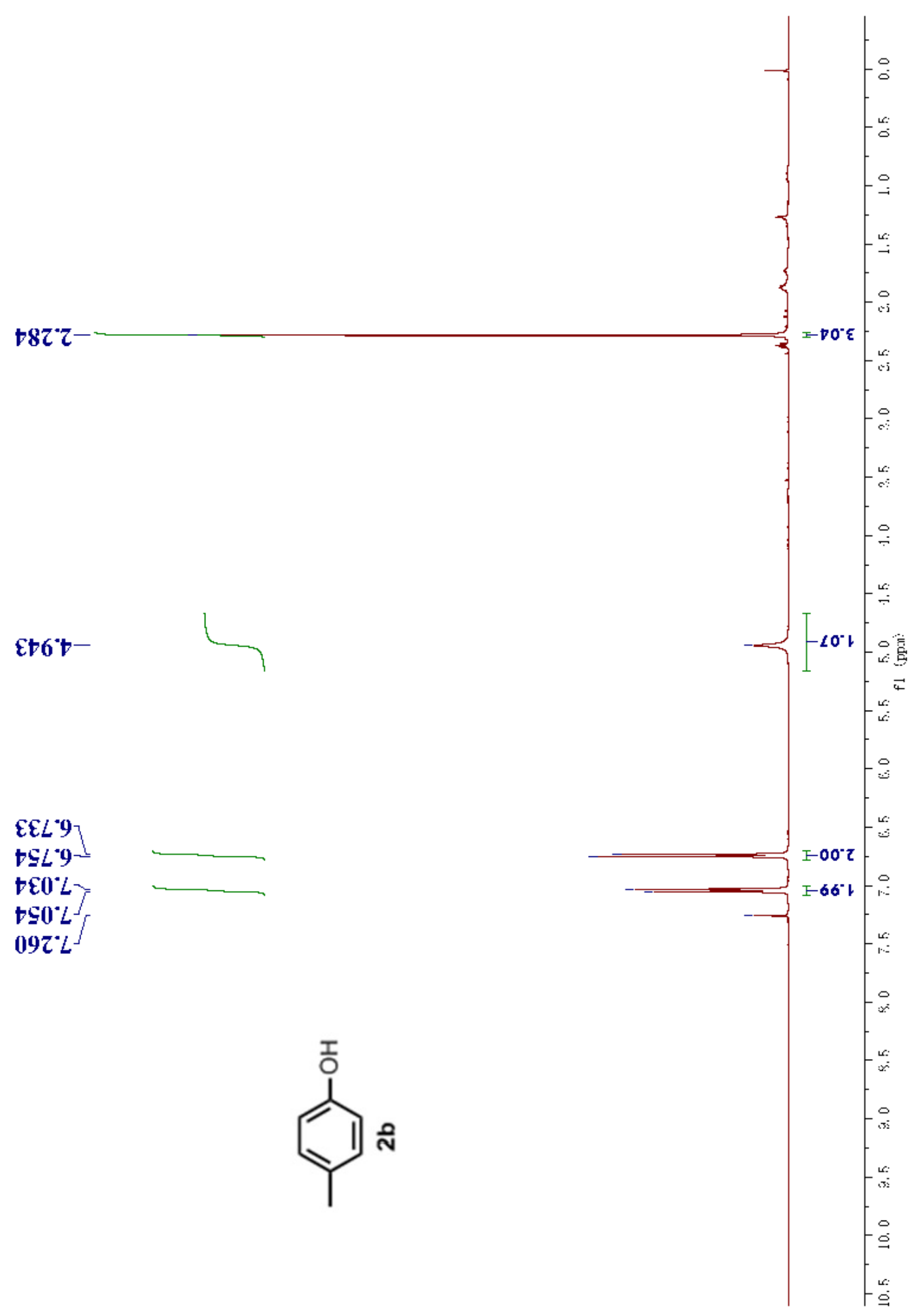


ssฐ0z-

$2+8.9 L$

$09 \mathrm{I}^{\circ} L L$

$\angle L T^{\circ} L L$

$\mathcal{\varepsilon Z Z ` S I - ~}$

$680^{\circ} 0 \varepsilon \mathrm{L}$

96 $\mathrm{L}^{\circ} 0 \varepsilon \mathrm{L}^{-}$

$\mathcal{E} \mathcal{E} \varepsilon \subseteq \mathrm{I}-$<smiles>Cc1ccc([O-])cc1</smiles>

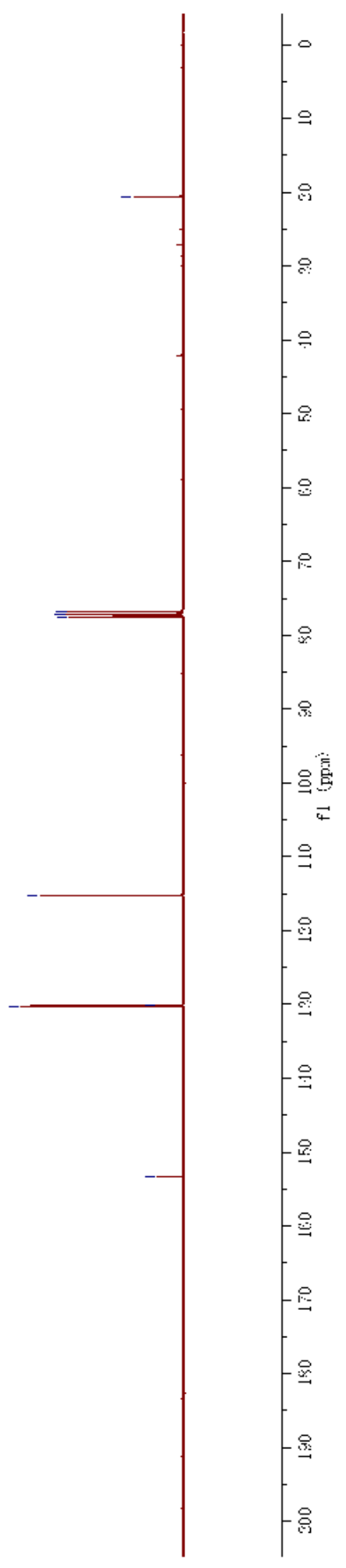




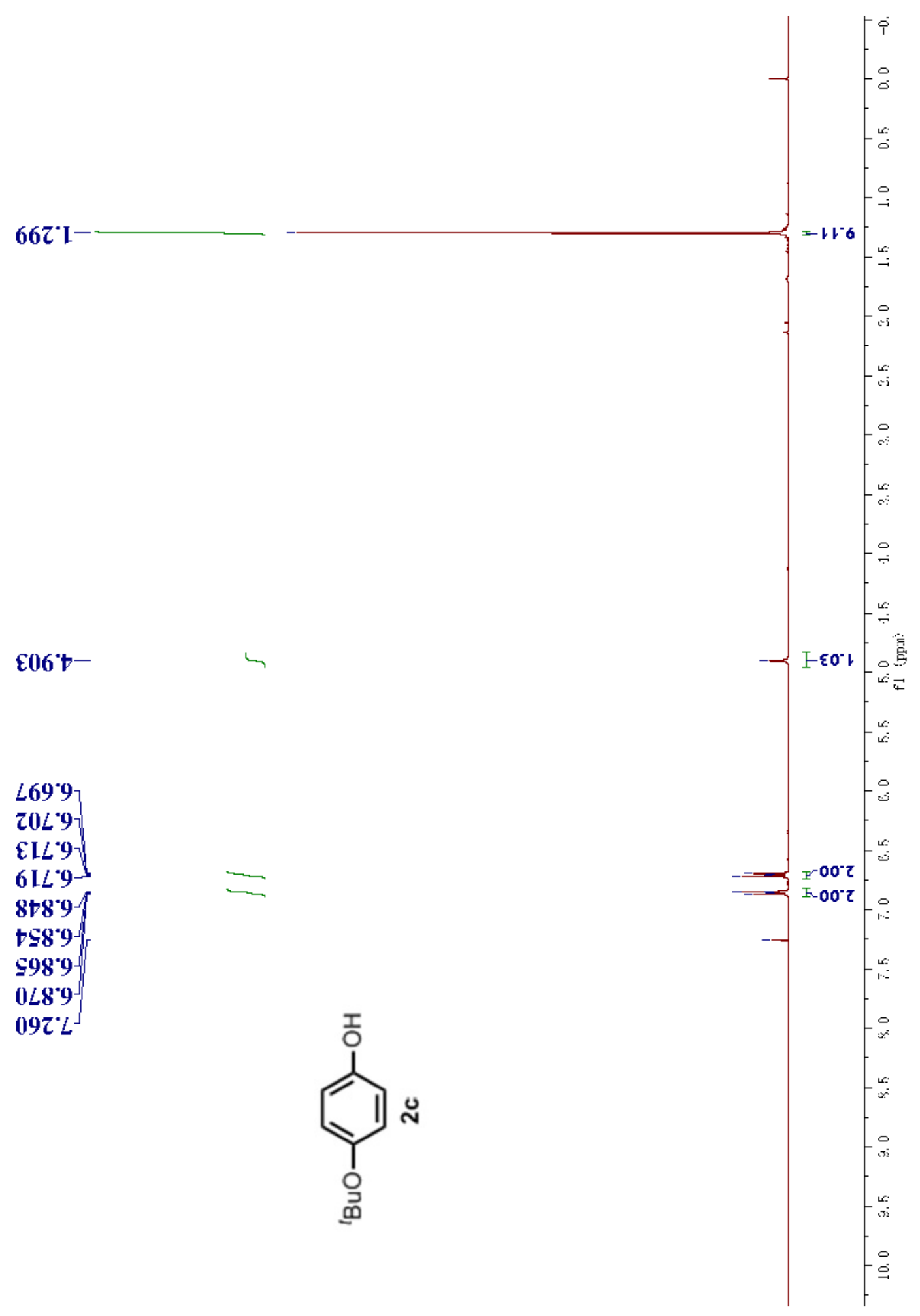




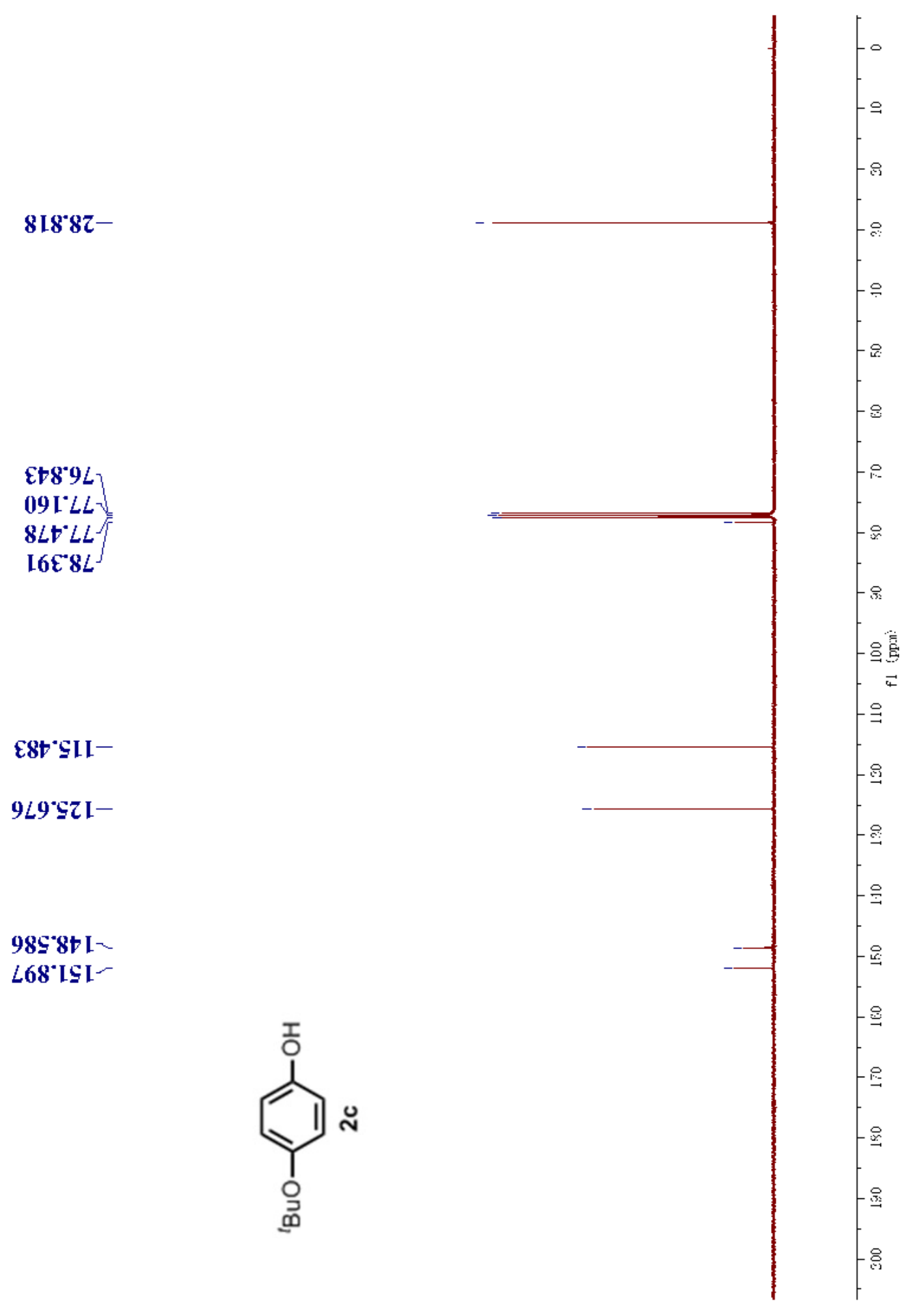




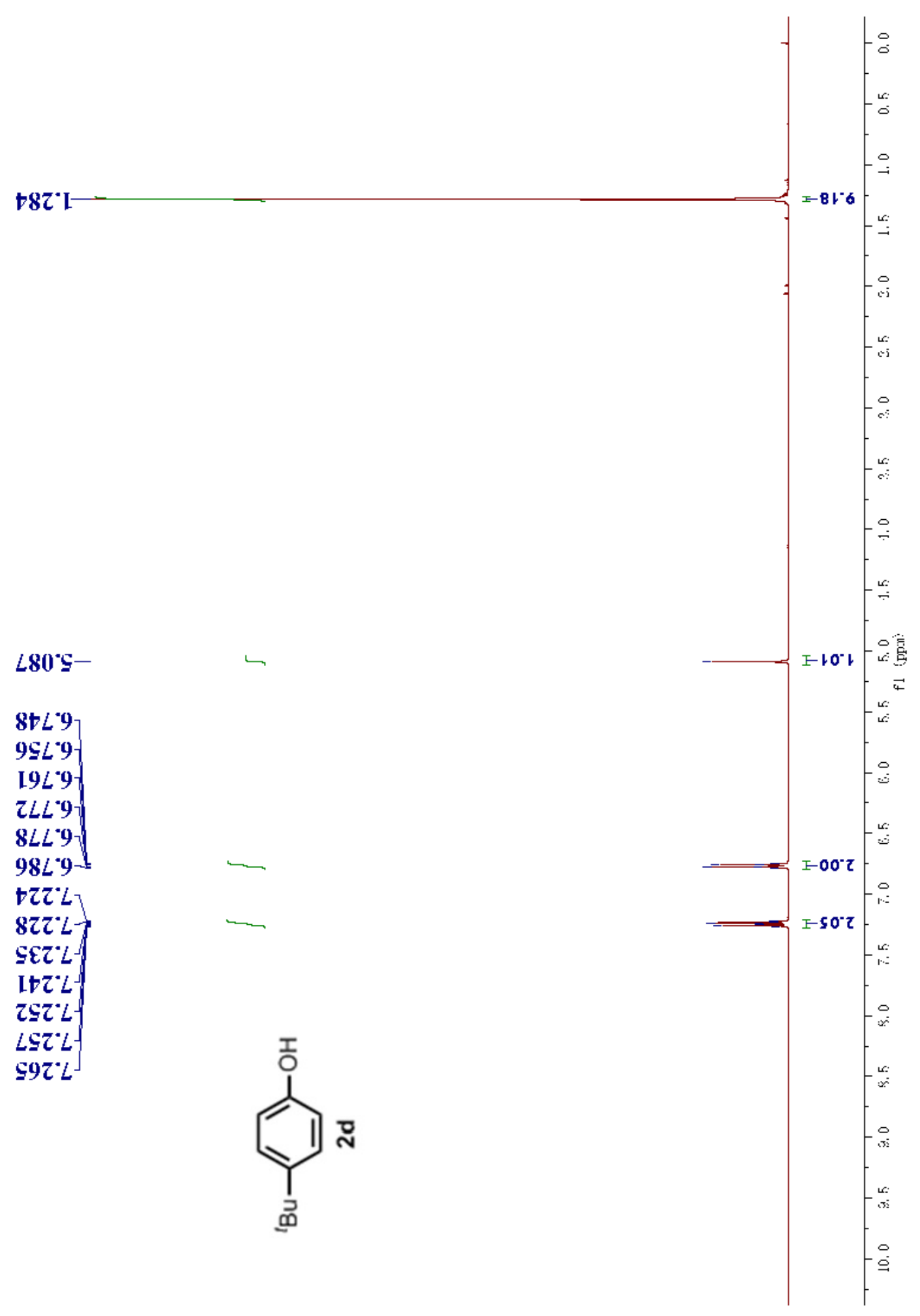




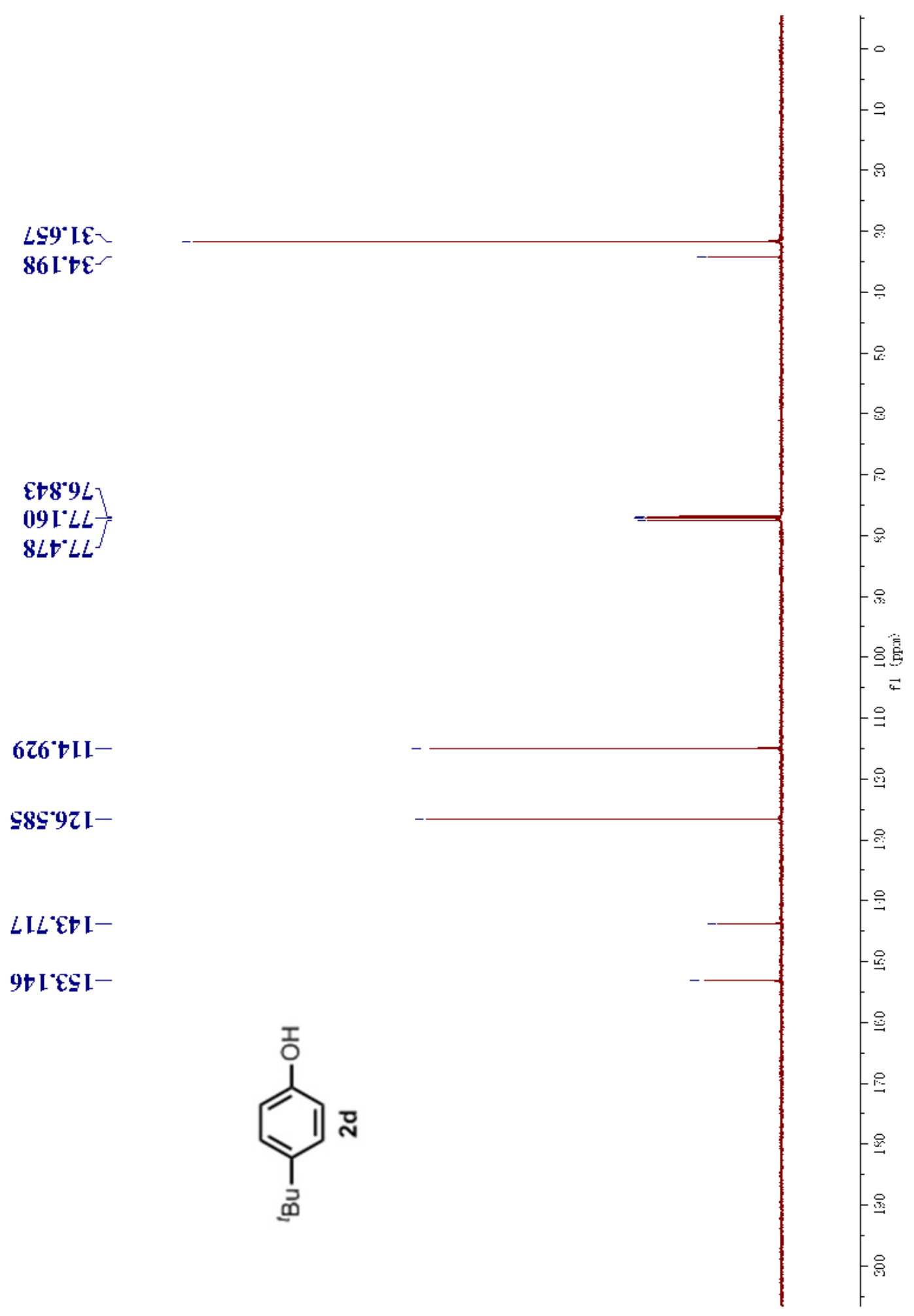




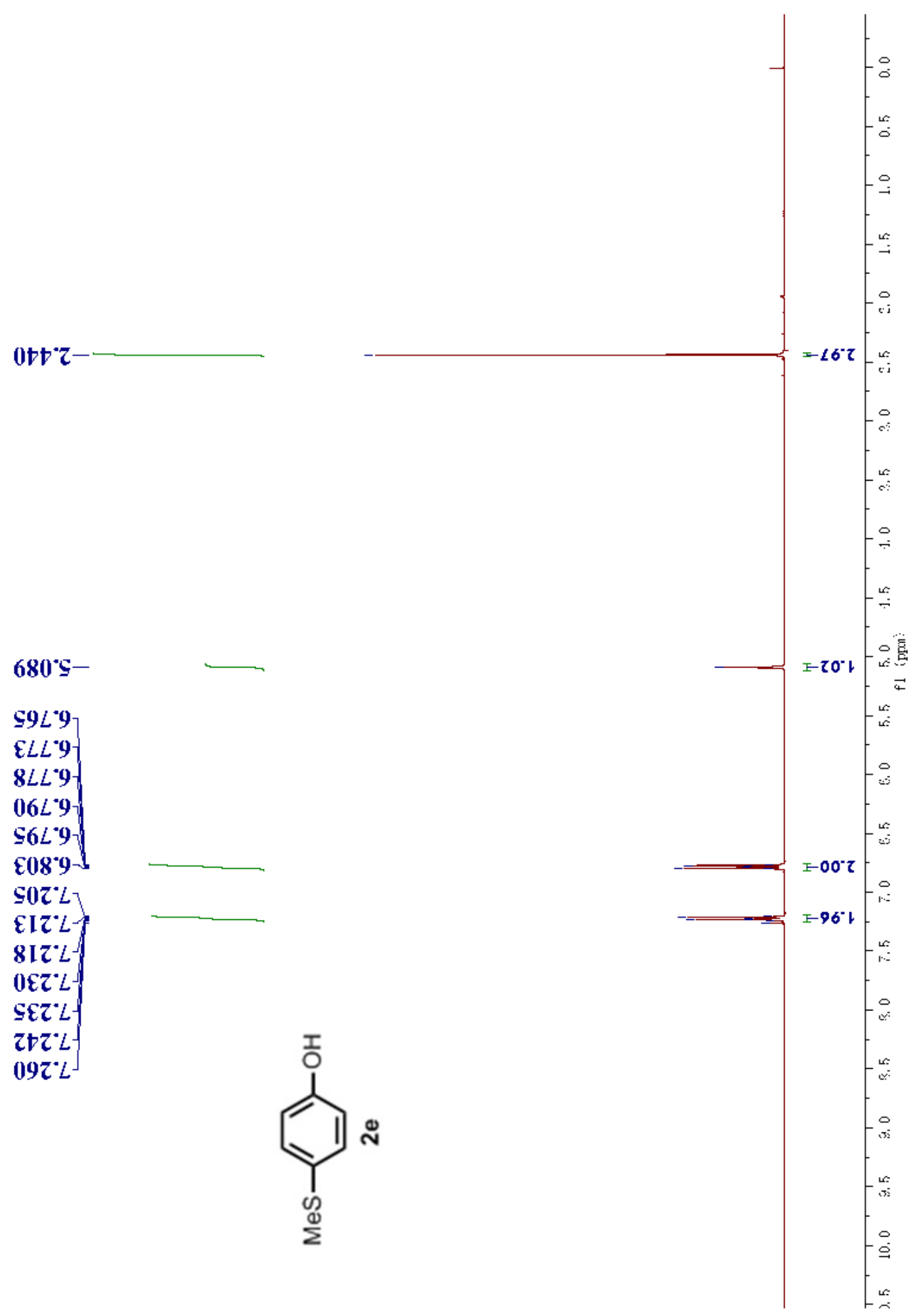


$99 I^{\circ} 8 \mathrm{I}-$

$258.9 L$

$09 \mathrm{I}^{\circ} L L=$

$L L T^{\circ} L L^{J}$

0Iz*9II-

$500 \% 2 \mathrm{I}$

ZIS'0EI ${ }^{\checkmark}$

ESI'tSI-

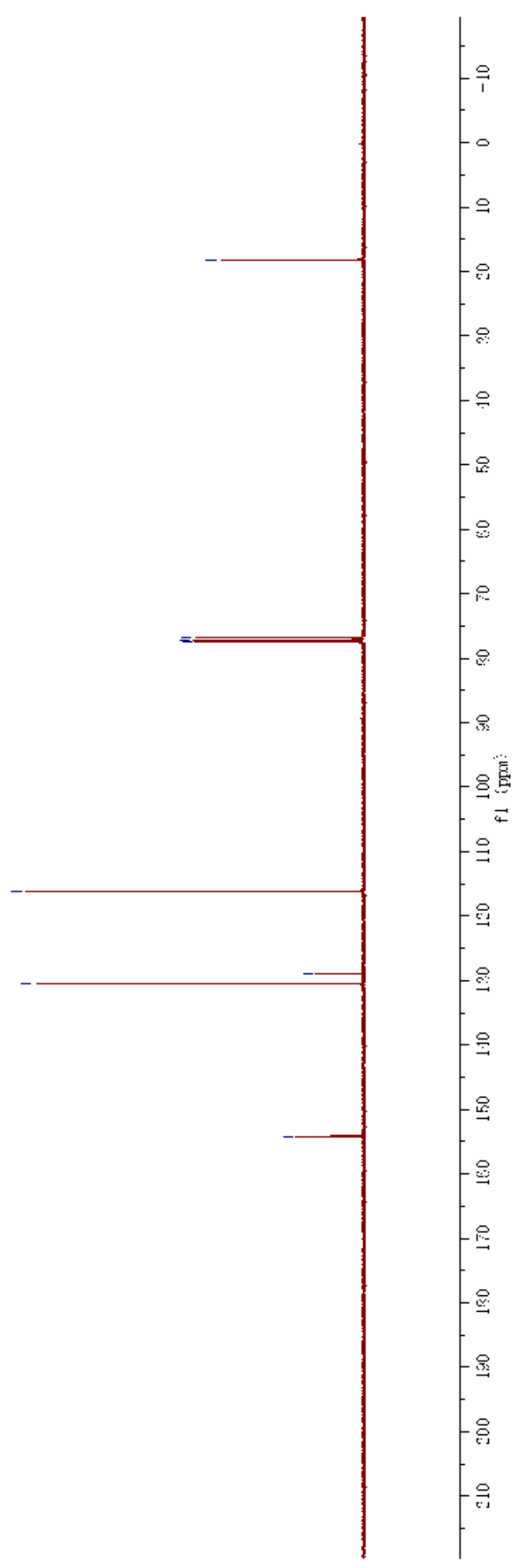


$\left.\begin{array}{l}96 t^{\circ} z \\ 00 \varsigma^{2} z \\ \text { t0ভ } z\end{array}\right]$

[98\%

$288^{\circ} 9$

$\angle t z ' L$

$\$ 92:$

$E 8{ }^{\circ} L$

$L L \varepsilon^{*} L$

$96 \varepsilon^{\circ} \mathrm{L}$

SIt $L$

$\varepsilon \angle \sigma^{\circ} L$

$S 60^{\circ} L$

$\operatorname{ss} L$

$+\angle S L$

$\operatorname{ses} 6-$

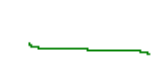

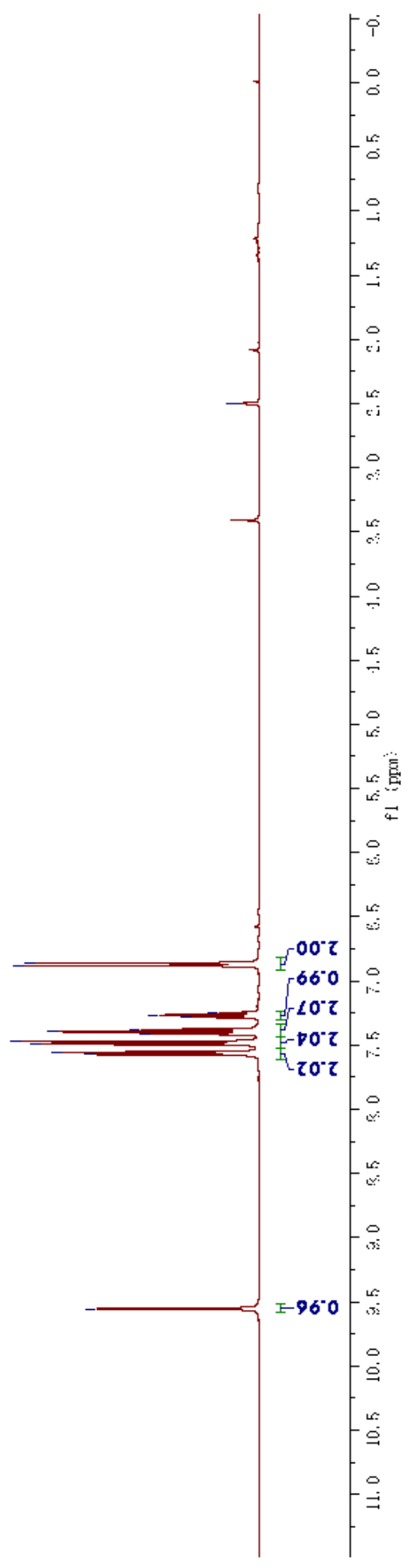




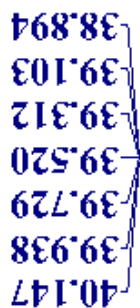

$\angle t L S I I-$

$096 \subseteq Z$ I

$\angle \downarrow E^{*} 9 Z \mathrm{I}$

$\angle E L^{\circ} L Z \mathrm{I}^{-}$

Z6L'8Z I

$0 \subseteq 6^{\circ} 0 \varepsilon \mathrm{I}^{\mathrm{J}}$

It $\boldsymbol{z}^{\circ} 0 \boldsymbol{t} \mathrm{I}$

ISI $L S I-$

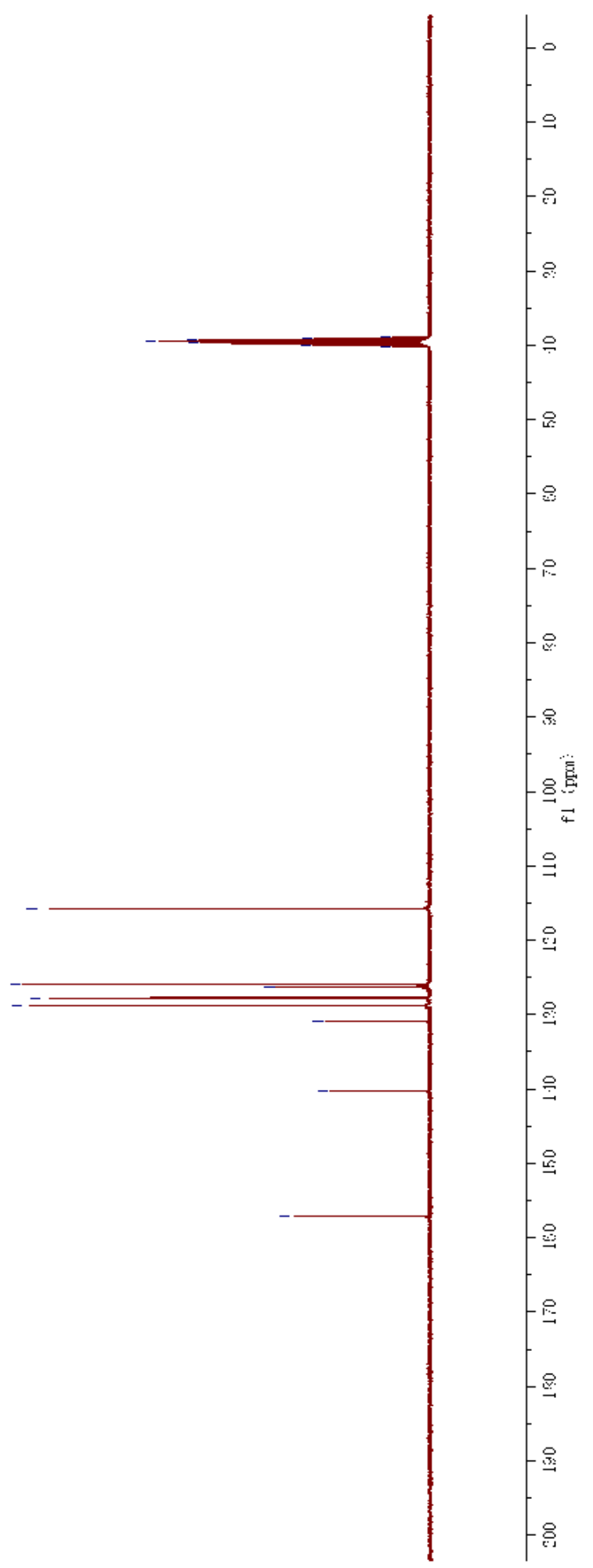


I6t"

$960^{\circ} \mathrm{z}$

$00 s z$

$\operatorname{tos} z$

605

$S S L^{\circ} 9$

$29 \angle 9$

$\angle 9 \angle 9$

$6 L L 9$

$+8 \angle 9$

$06 L^{\circ} 9^{\circ}$

$890{ }^{\circ}$

$S\left[L^{\circ} L\right.$

$z z L^{\circ} L$

$92 L L$

$6 \varepsilon L L$

$\varepsilon t L L L$

$0 S L L$

$626^{\circ} 6^{-}$

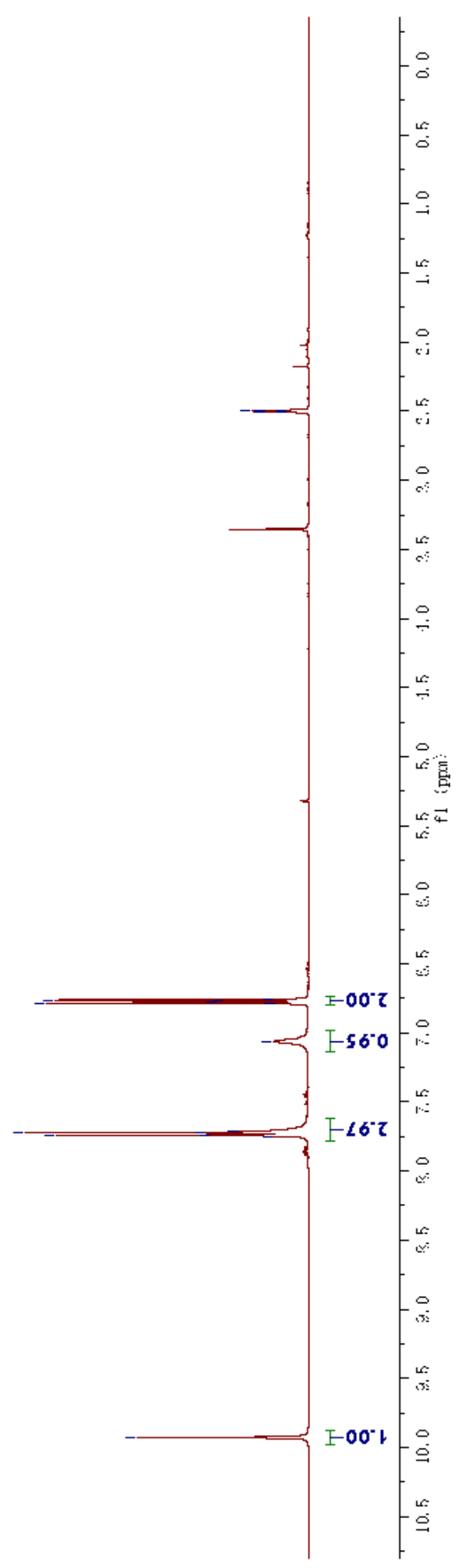




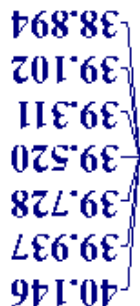

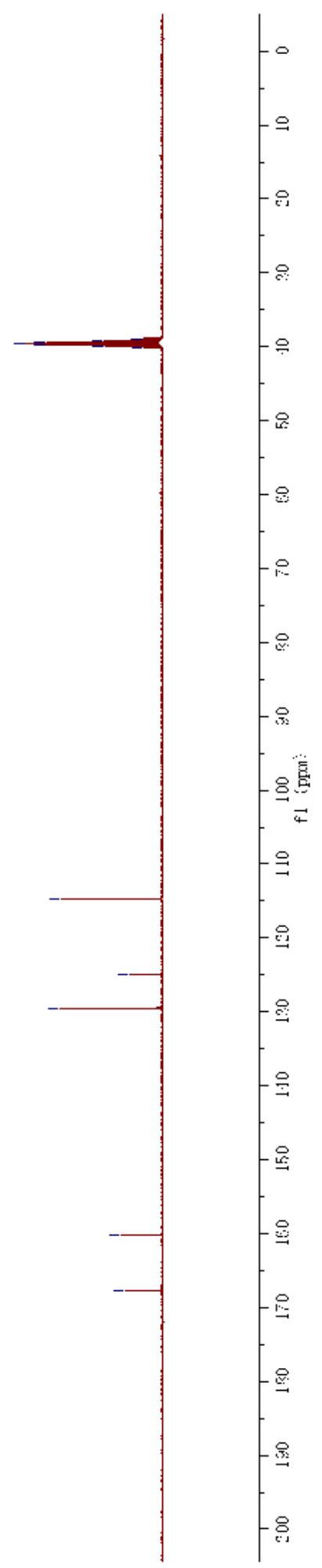




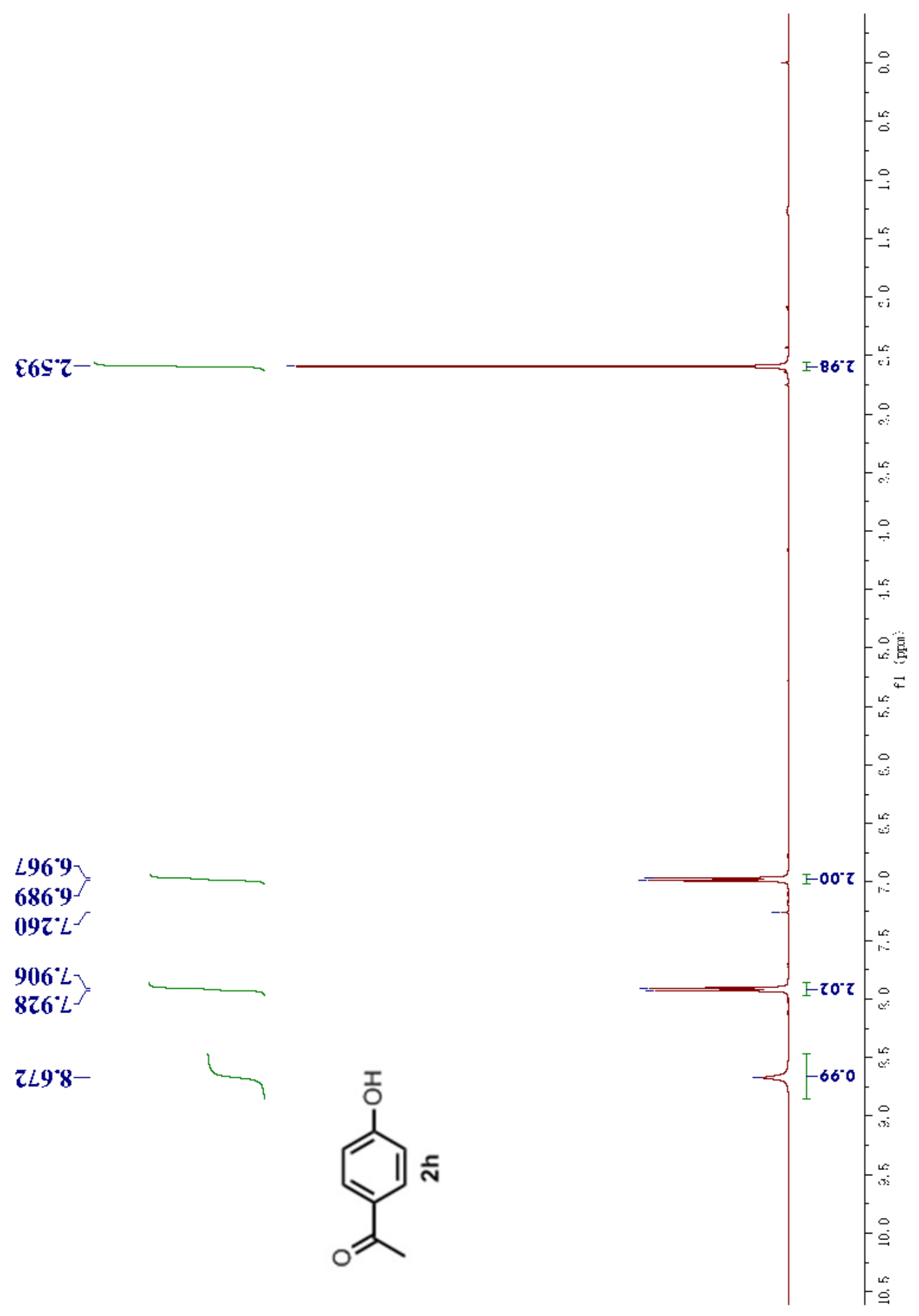


L9E9Z-

$\operatorname{ct8} 9 L$

$09 \mathrm{I}^{\circ} L L C$

$8 L 5^{\circ} L L$

\$9 $L^{\circ}$ SII-

$\varepsilon z \varepsilon^{*} 6 z I$

$\subseteq \Phi{ }^{*} I \varepsilon I^{-}$

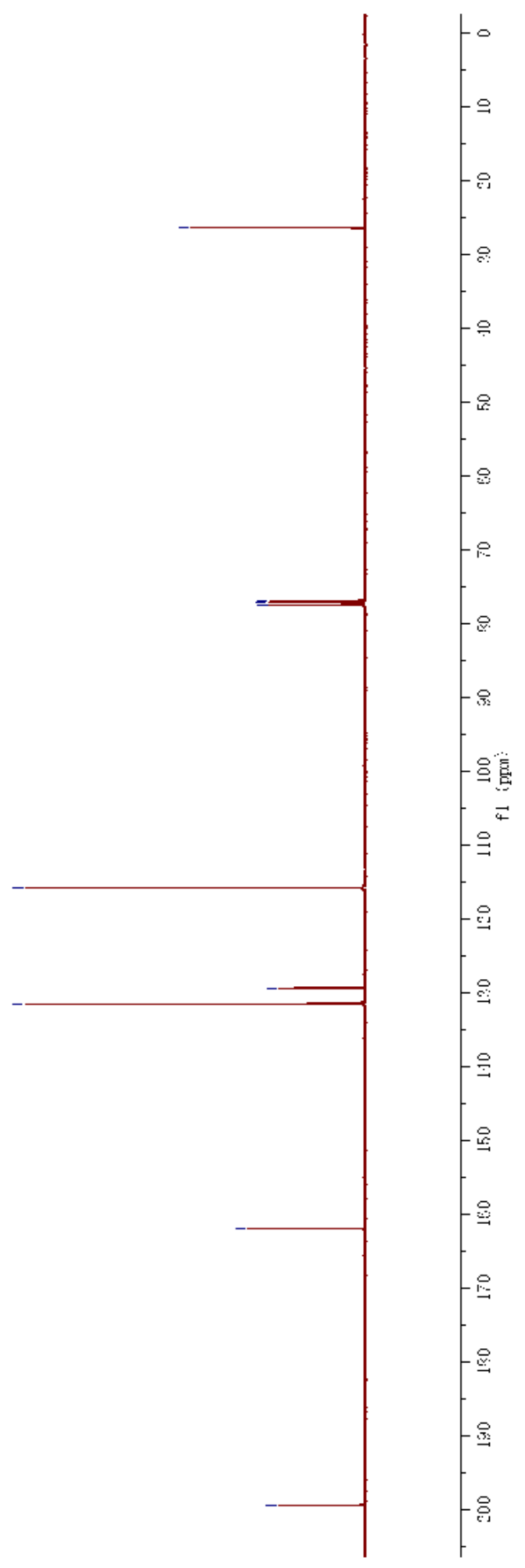




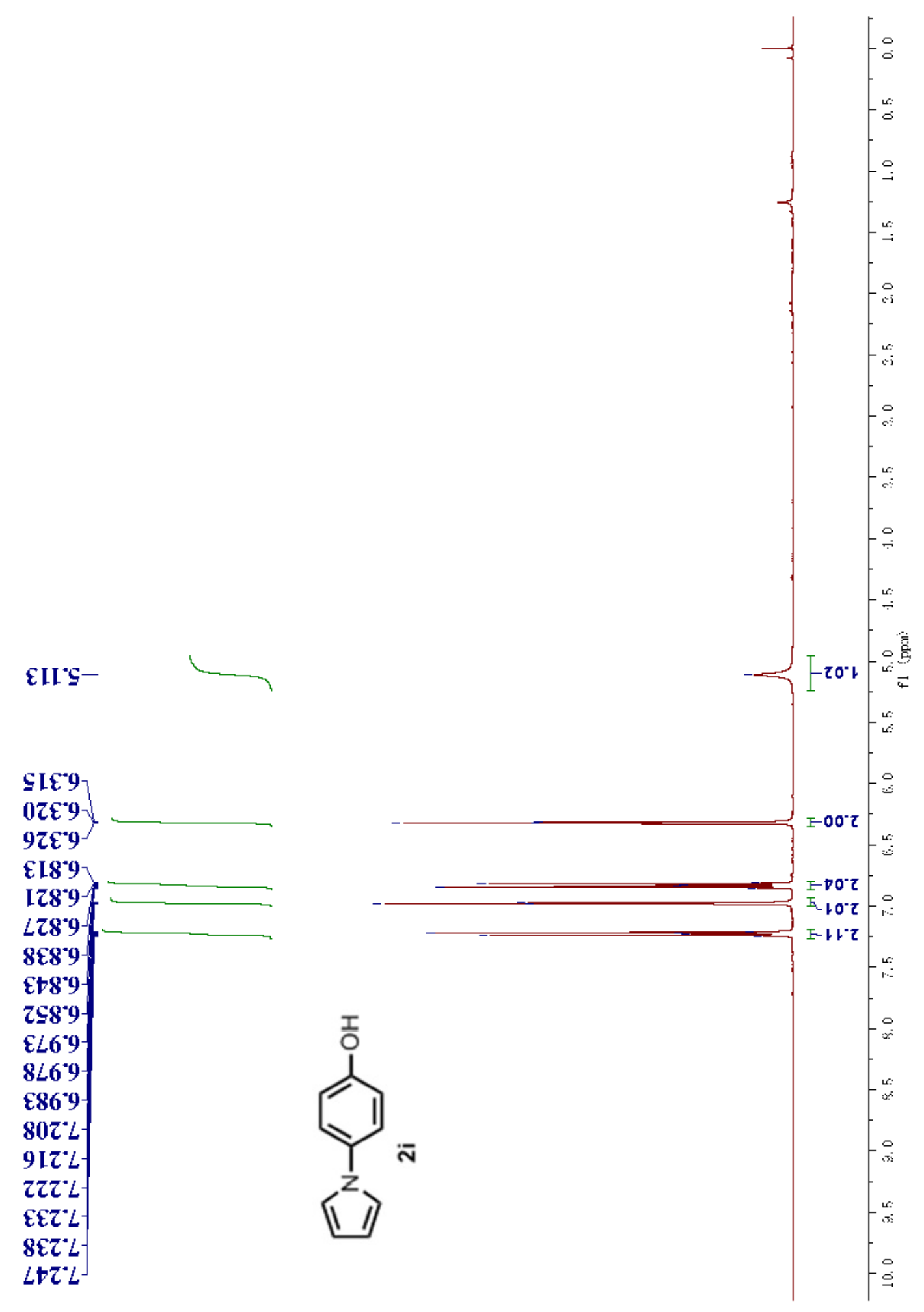




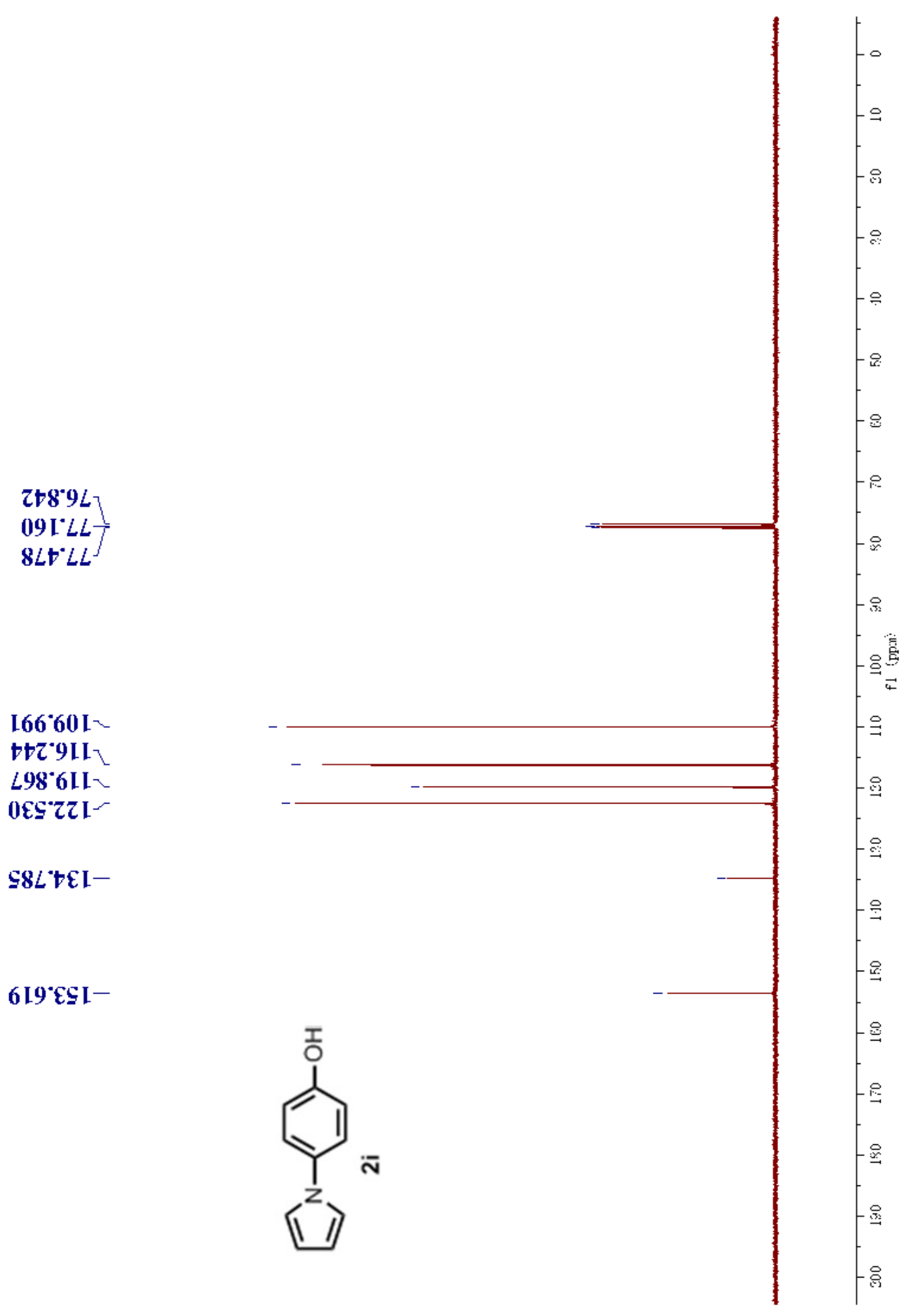




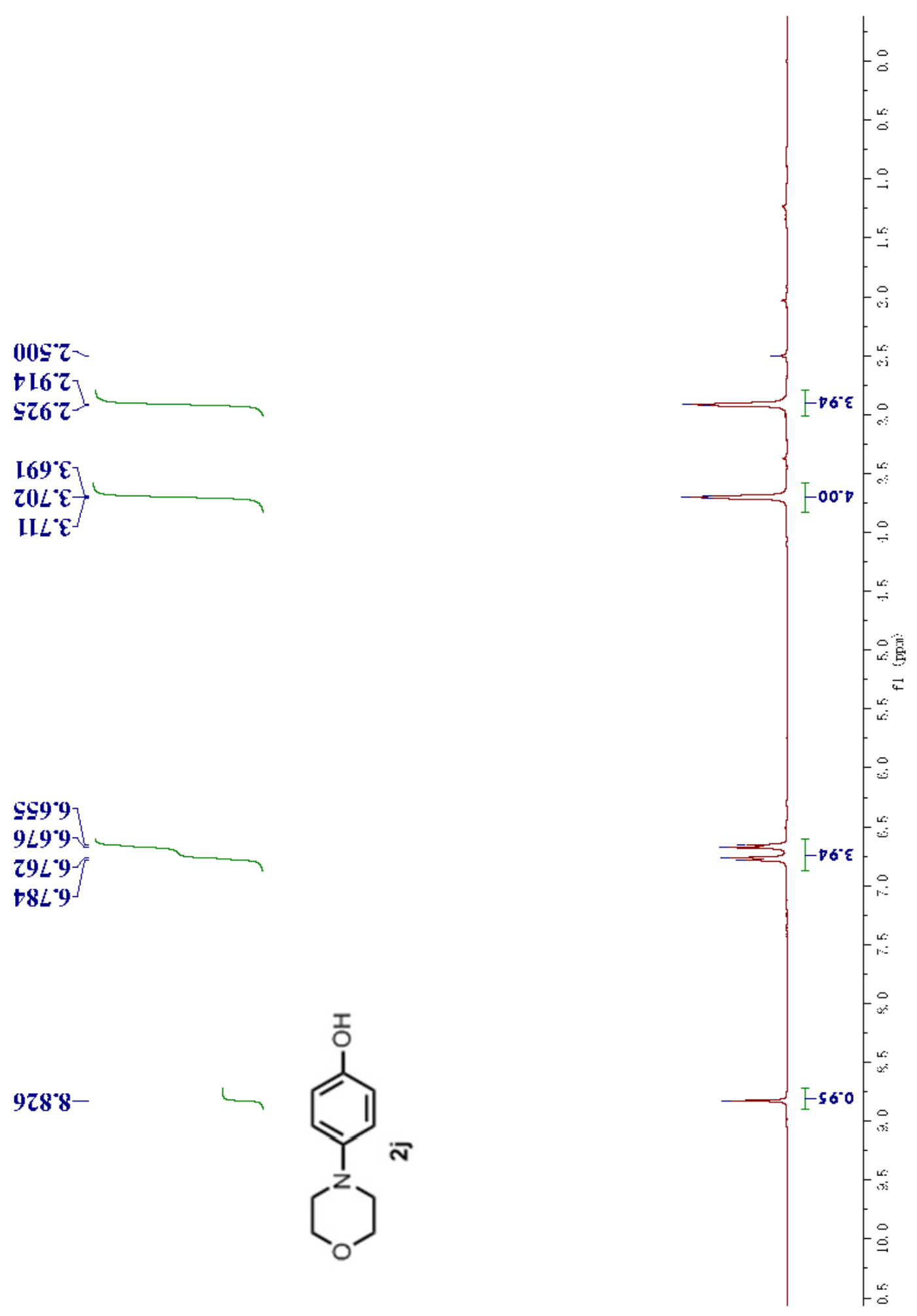




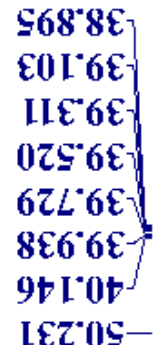

E82:99-

$88 t^{\circ} \subseteq$ Lᄂ

$+6 \varepsilon^{\circ} L \mathrm{LI}^{\prime}$

6LI'ttI-

$090^{\circ}$ LSI-<smiles>[CH2+]c1ccc([C]2CCOCC2)cc1</smiles>

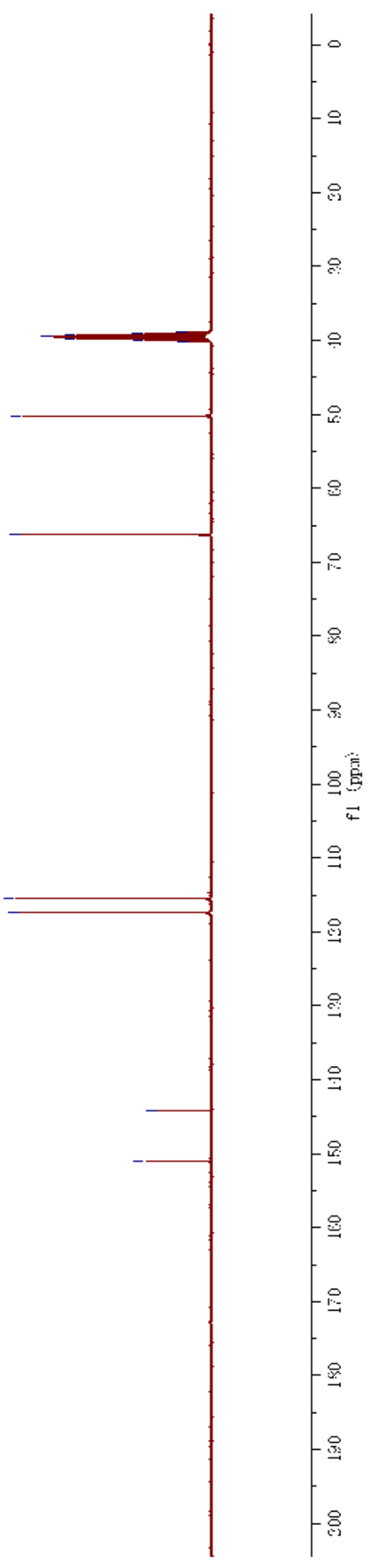




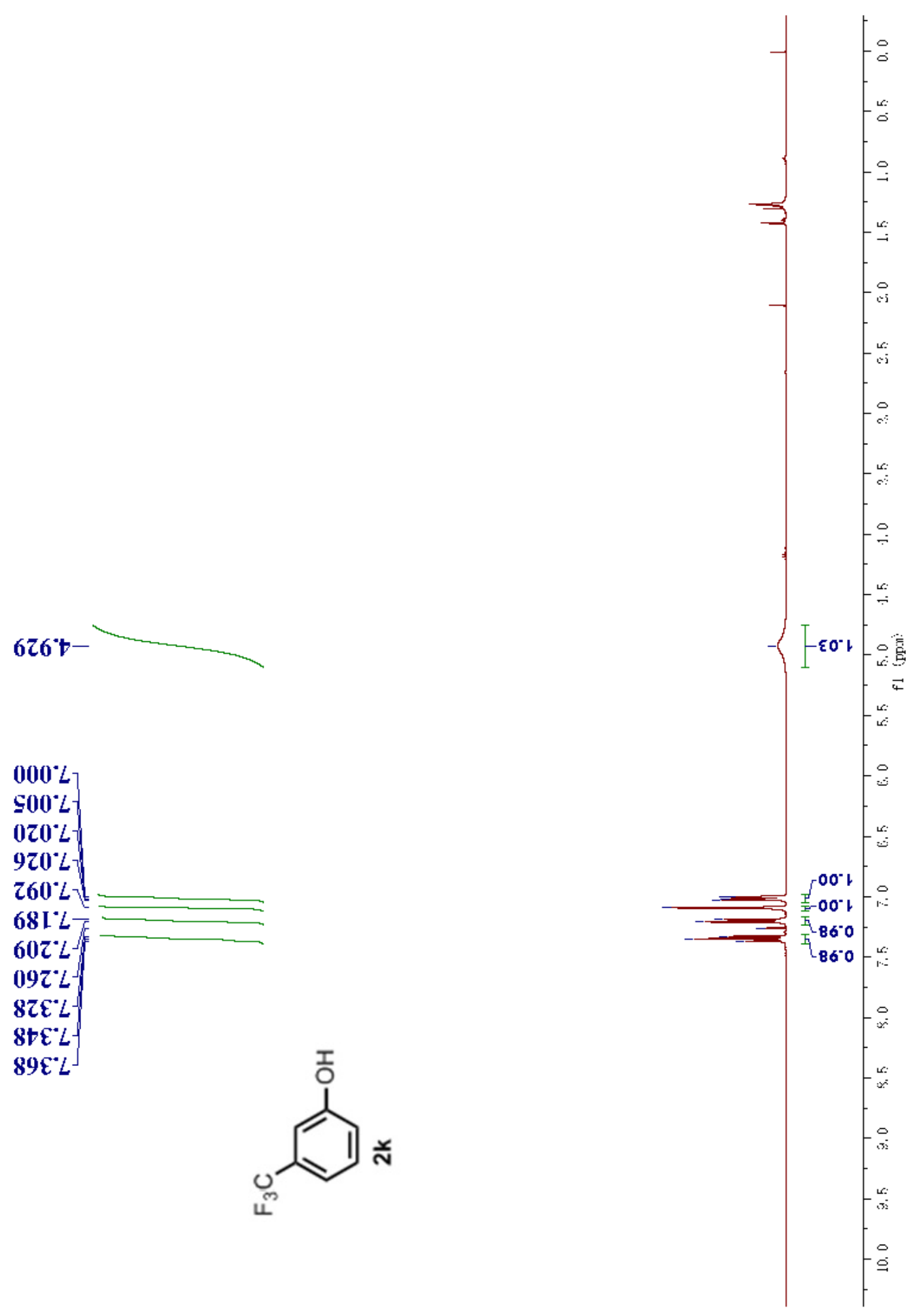



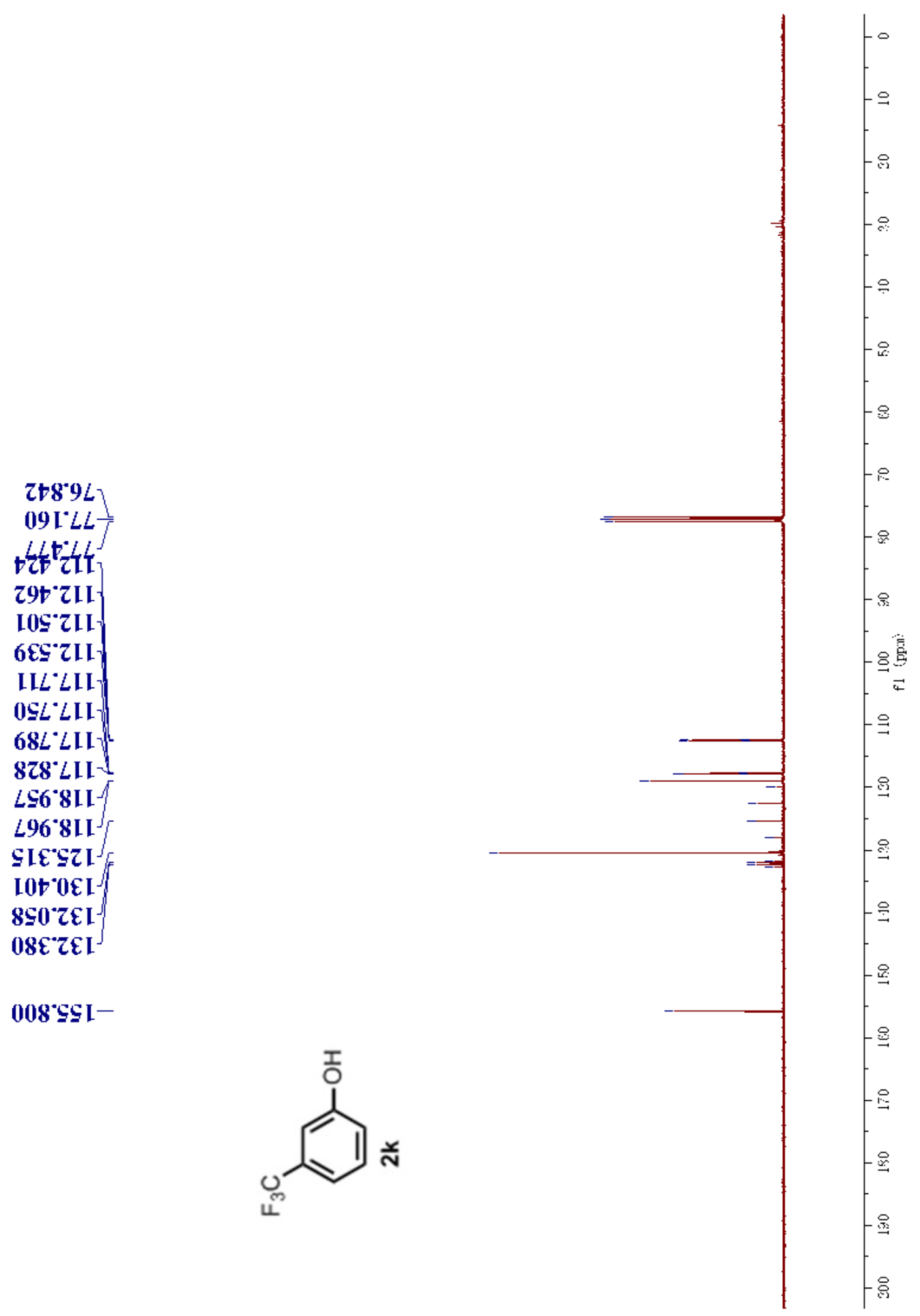


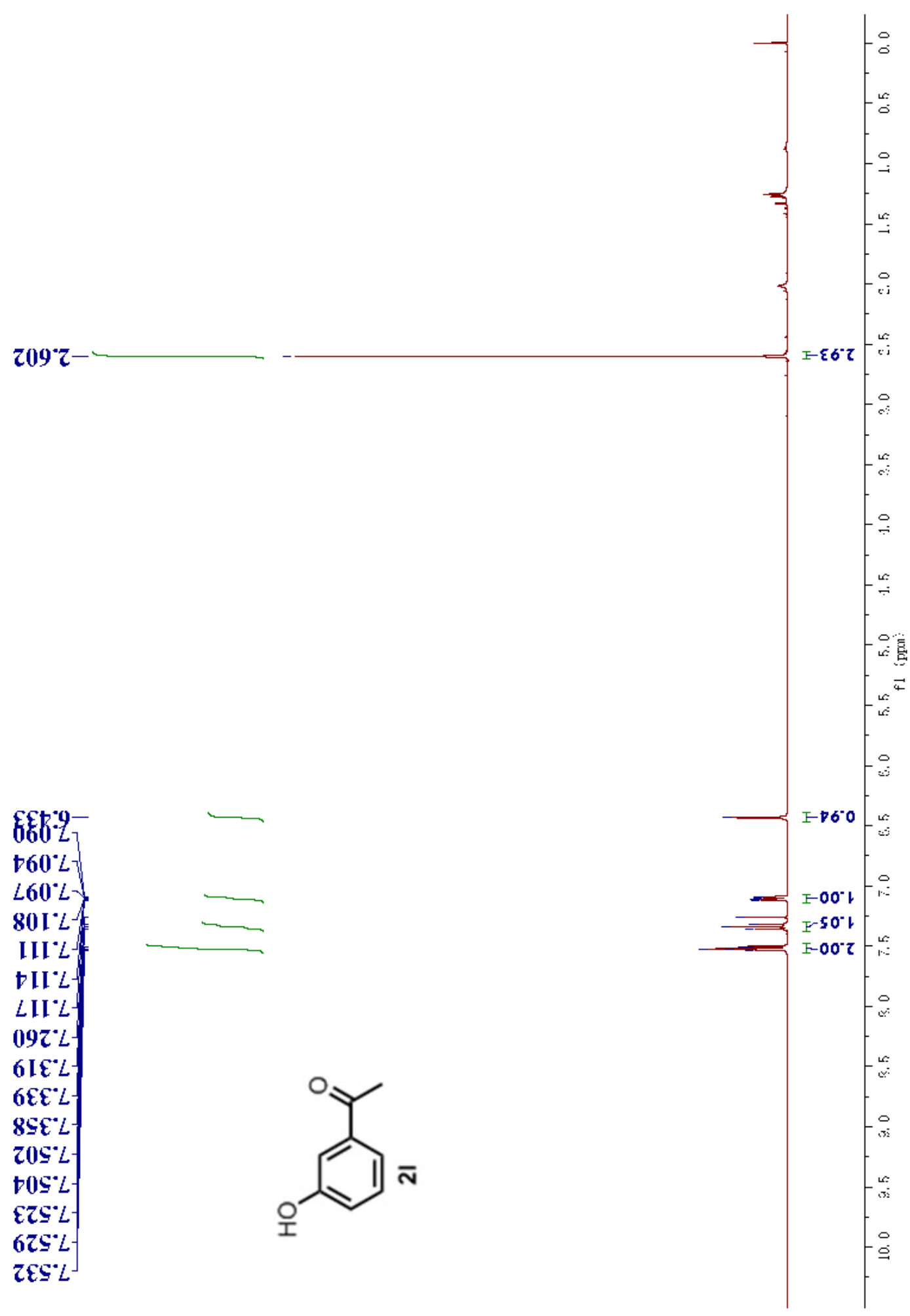


I06.97-

$258^{\circ} 9 L$

$09 \mathrm{I}^{\circ} L L-$

$L L T^{\circ} L L$

E $L 8^{\circ}$ tI I $^{-}$

$\angle S 0^{\circ} I Z I^{\top} T$

6t I'IZI

$\tau \rightarrow 0^{\circ} 0 \varepsilon \mathrm{I}^{-}$

S6E $8 \varepsilon \mathrm{I}$.

$86 \mathrm{~S}^{\circ} 9 \mathrm{I}-$

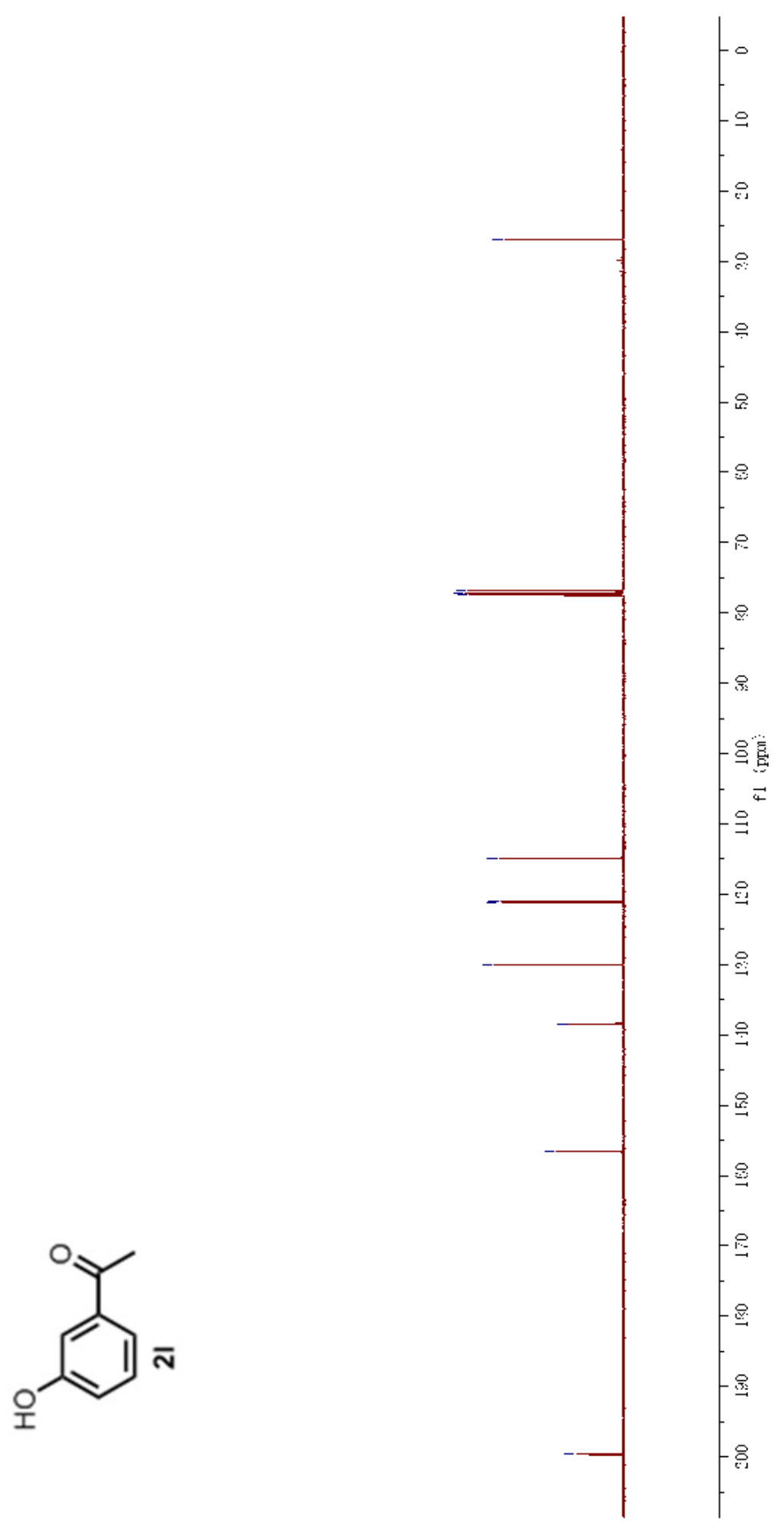




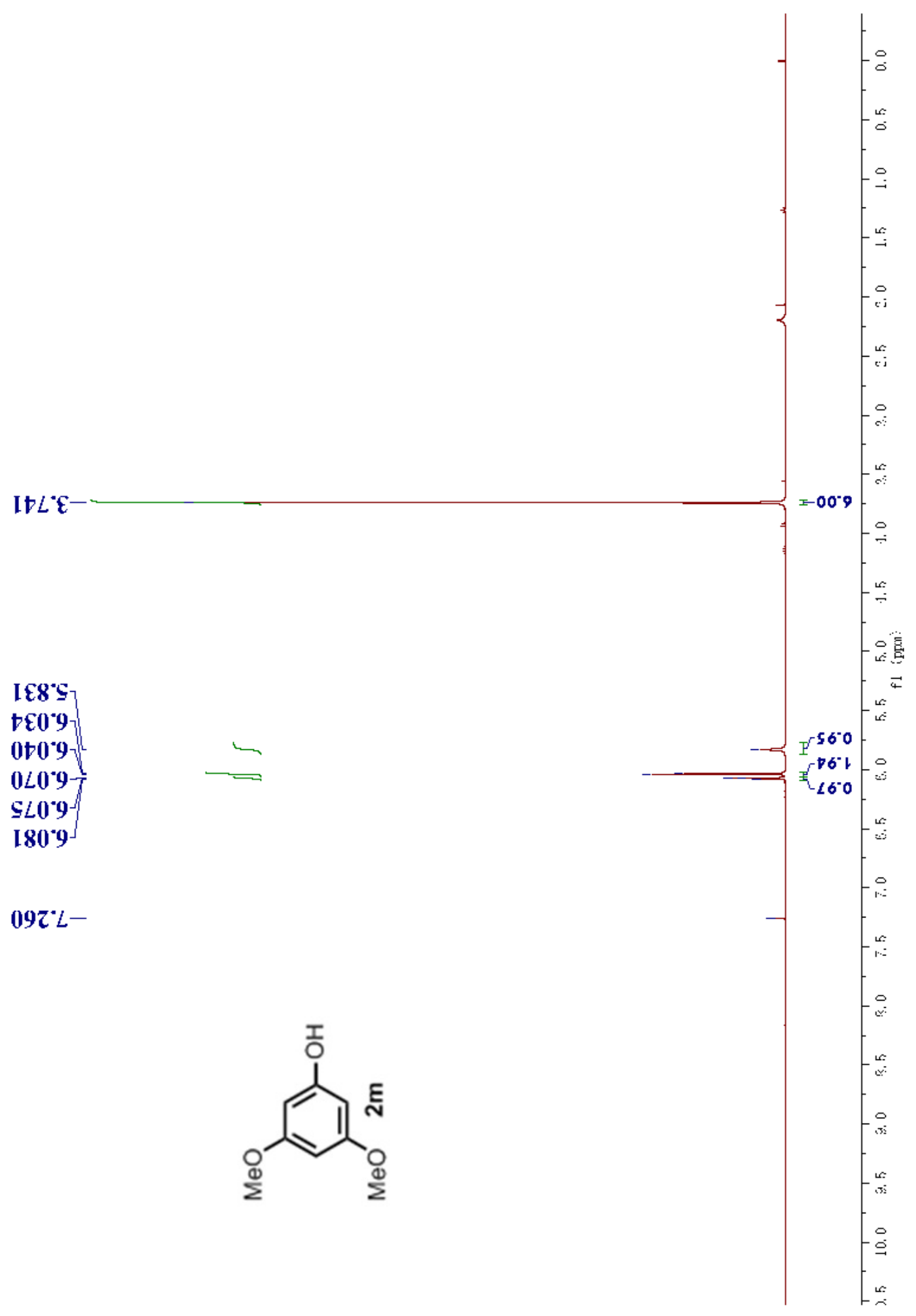




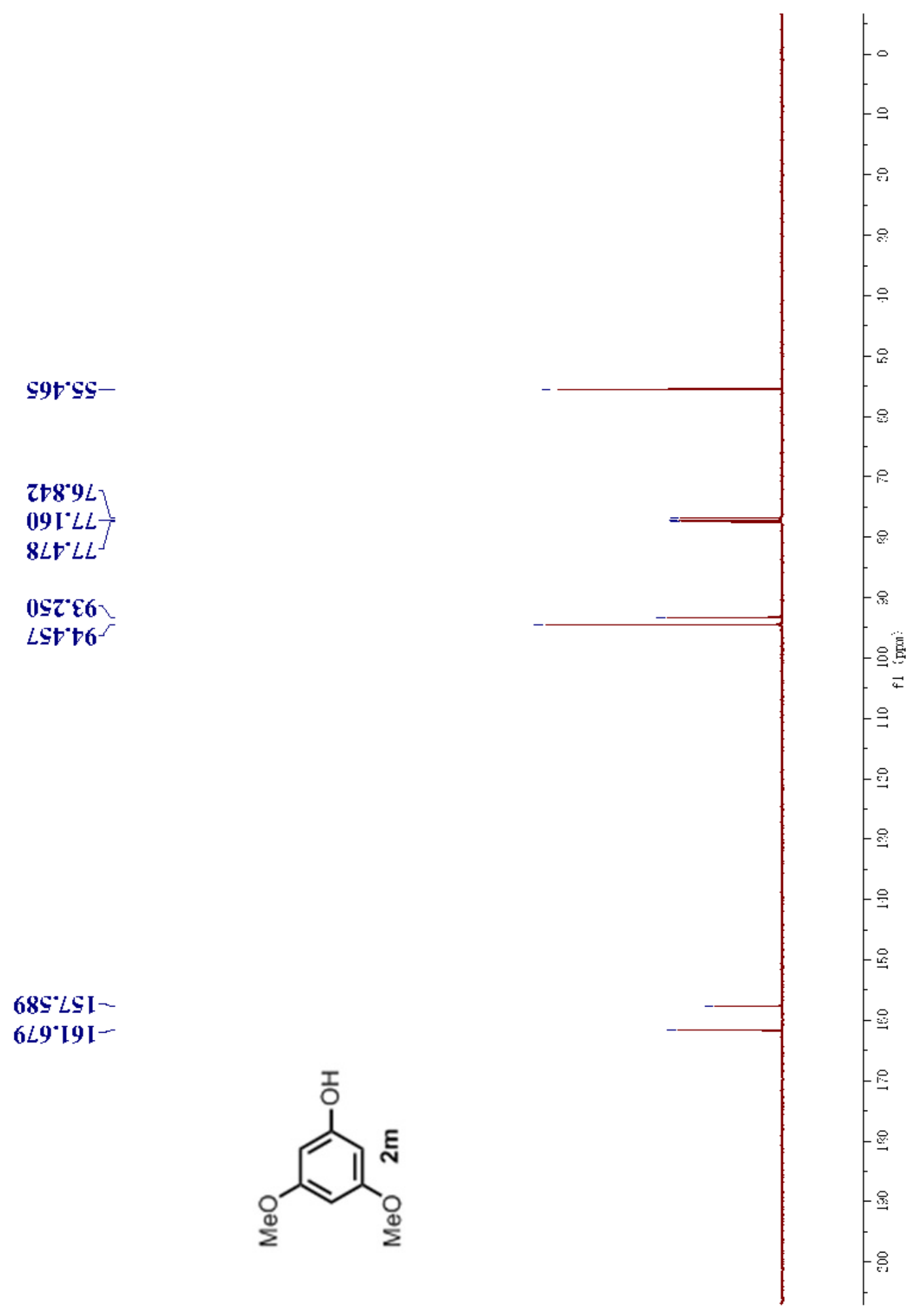




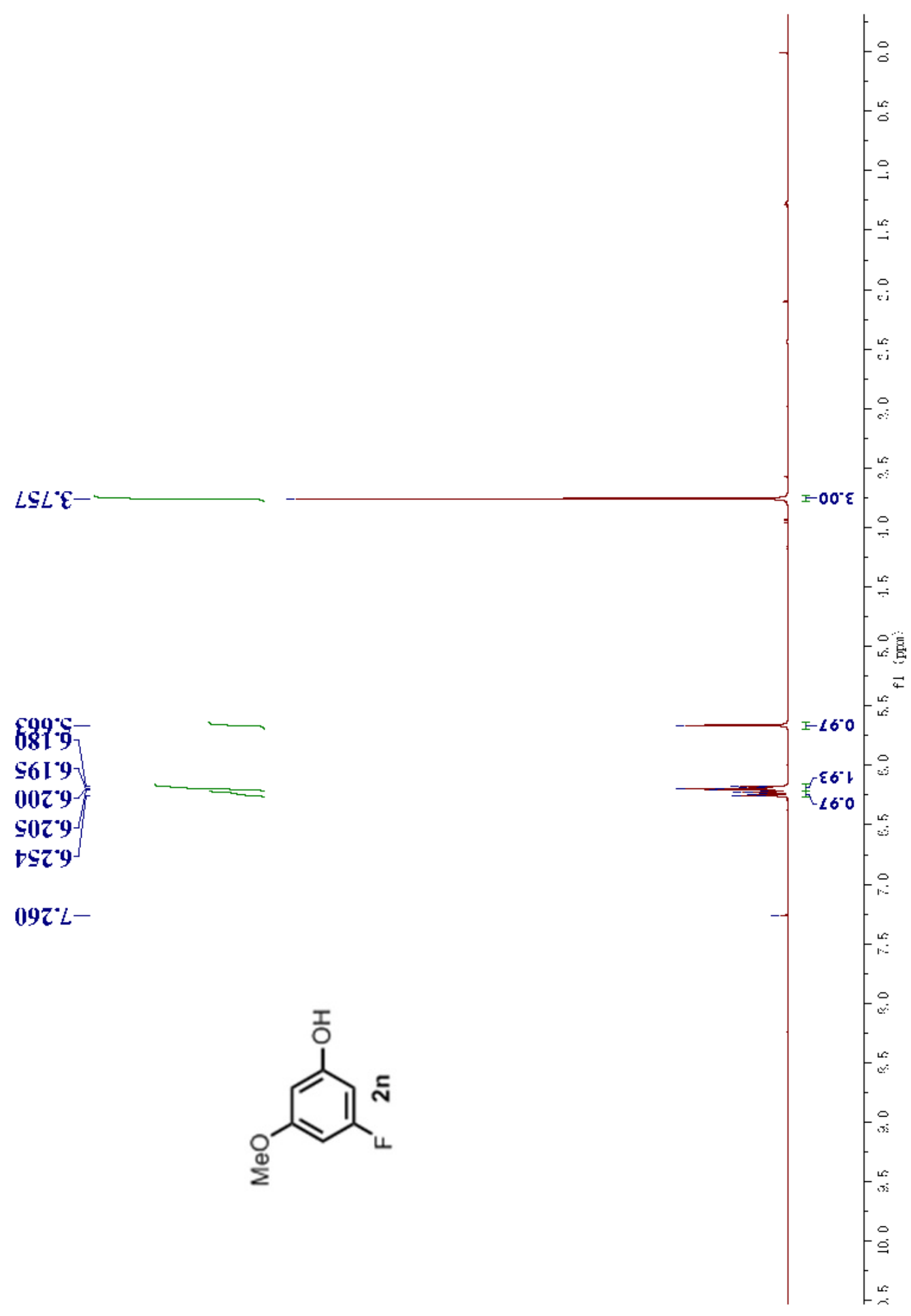




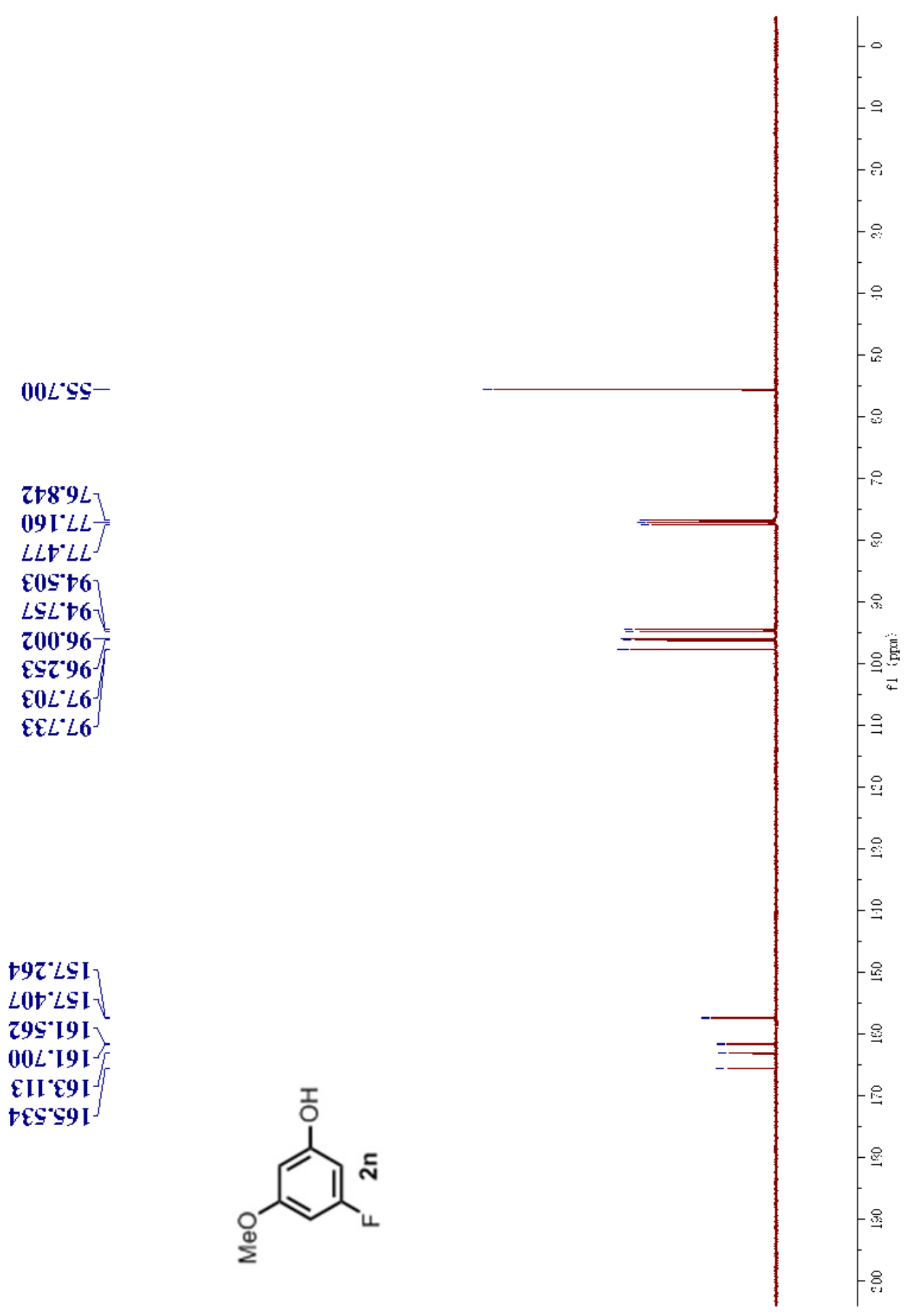




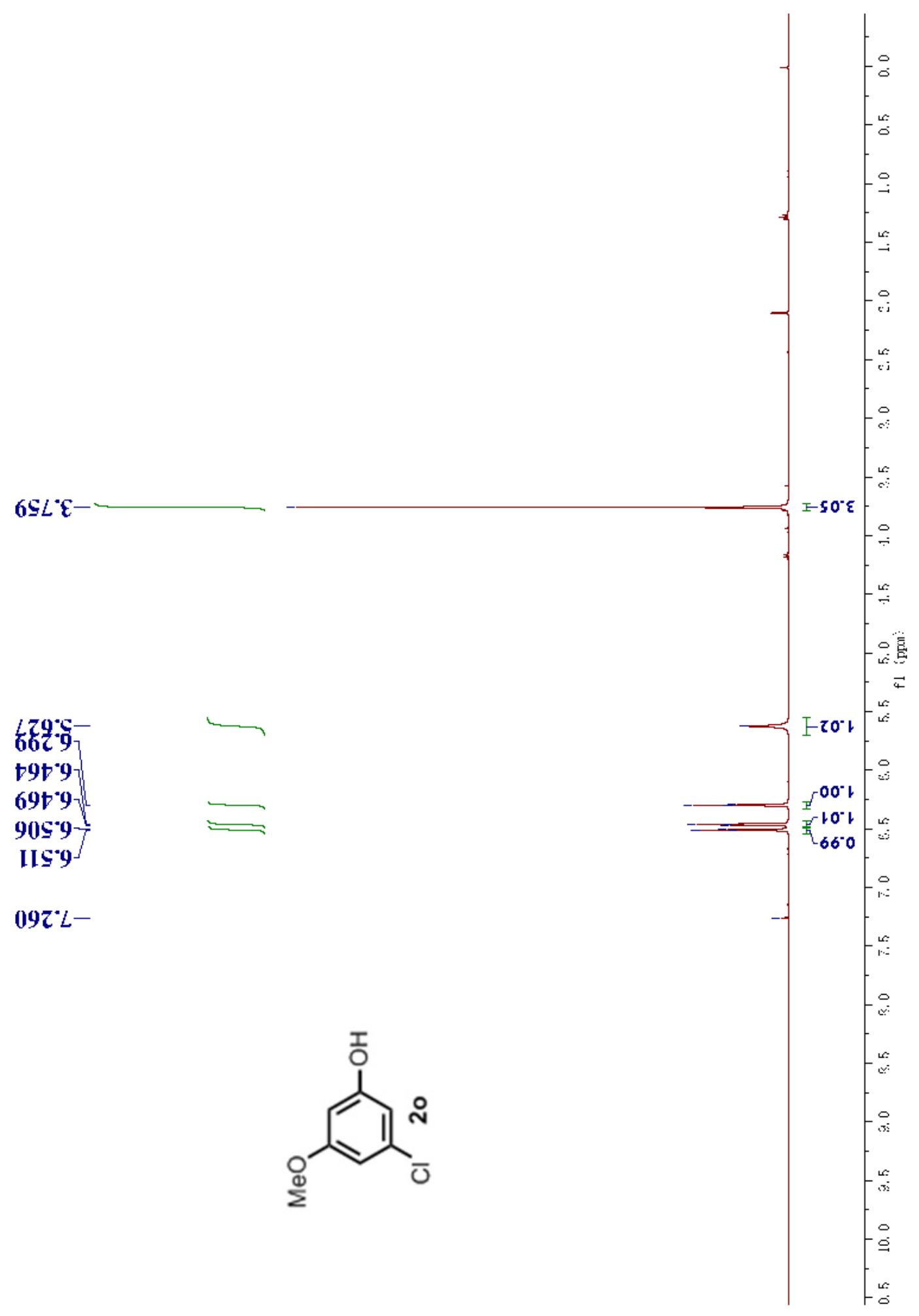




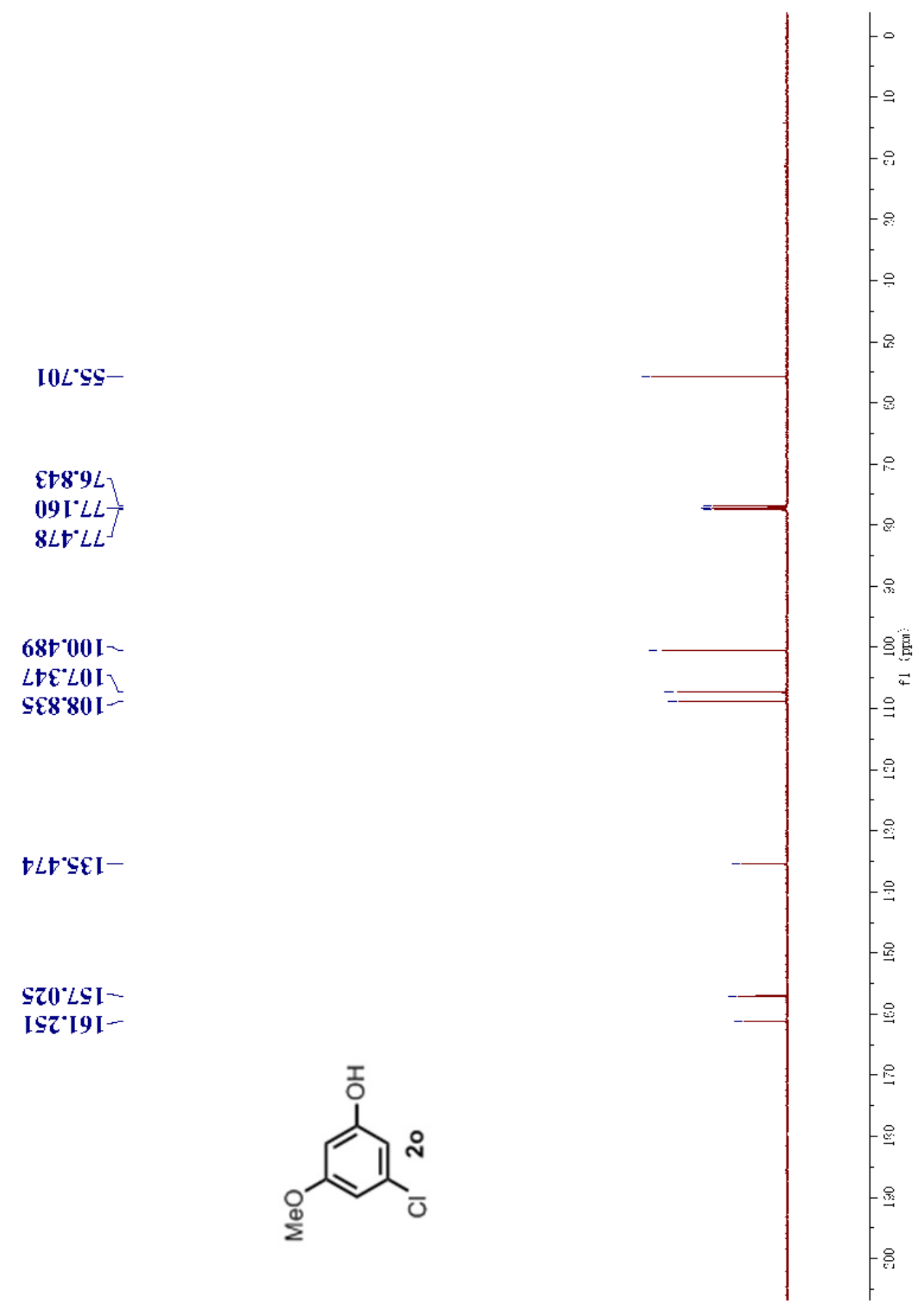




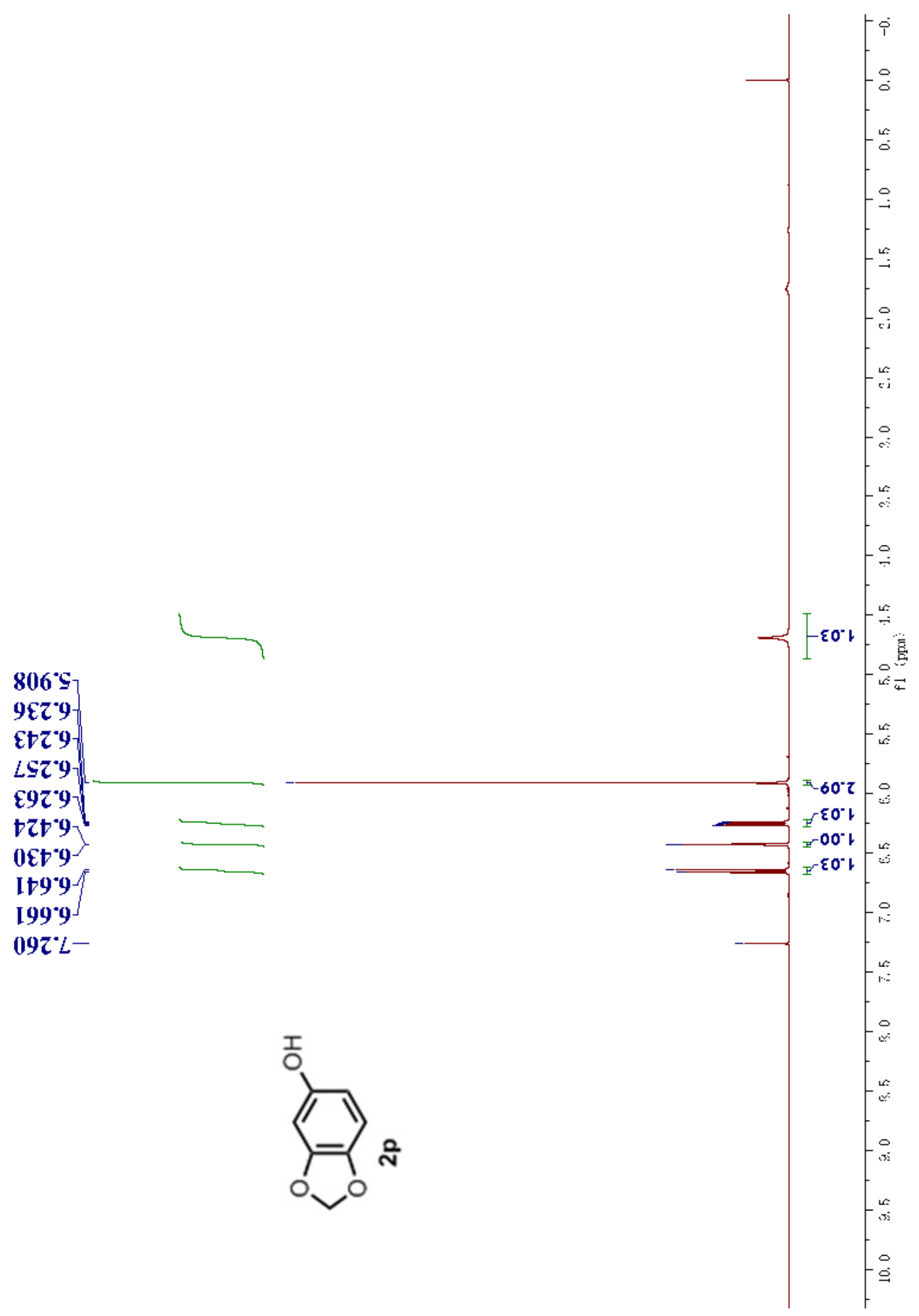


$258^{\circ} 9 L$

$09 \mathrm{I}^{\circ} L L=$

$8 L t^{\circ} L L$

$0 \varepsilon t^{\circ 6}$ -

6LI'I0I-

$\varepsilon 68^{\circ} 90 \mathrm{I}$

$\$ 62^{\circ} 80 \mathrm{I}^{\top}$

6IS'ItI-

$z \varepsilon z 8 \mathrm{~s}$ 【

99S0SI-

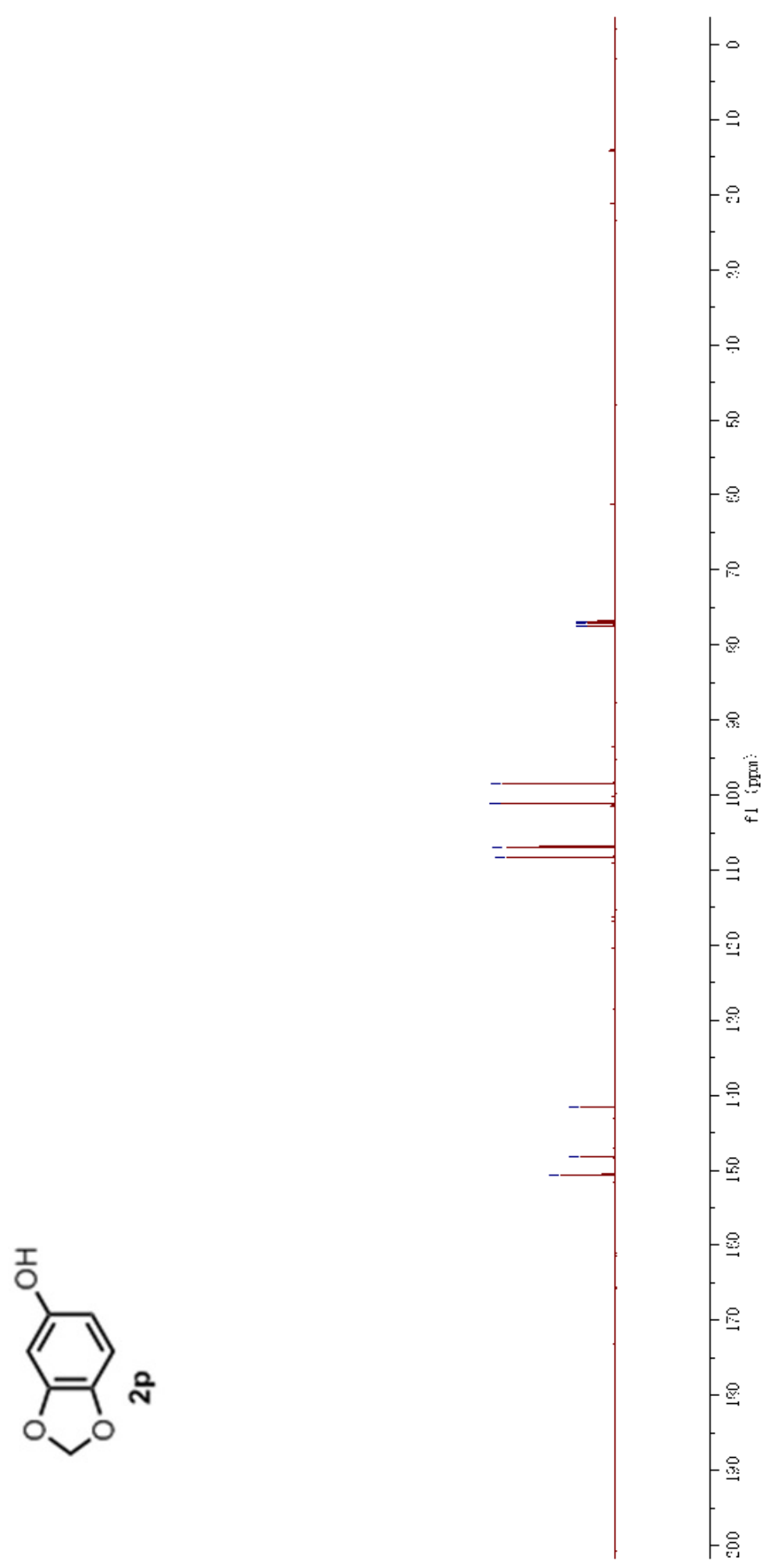




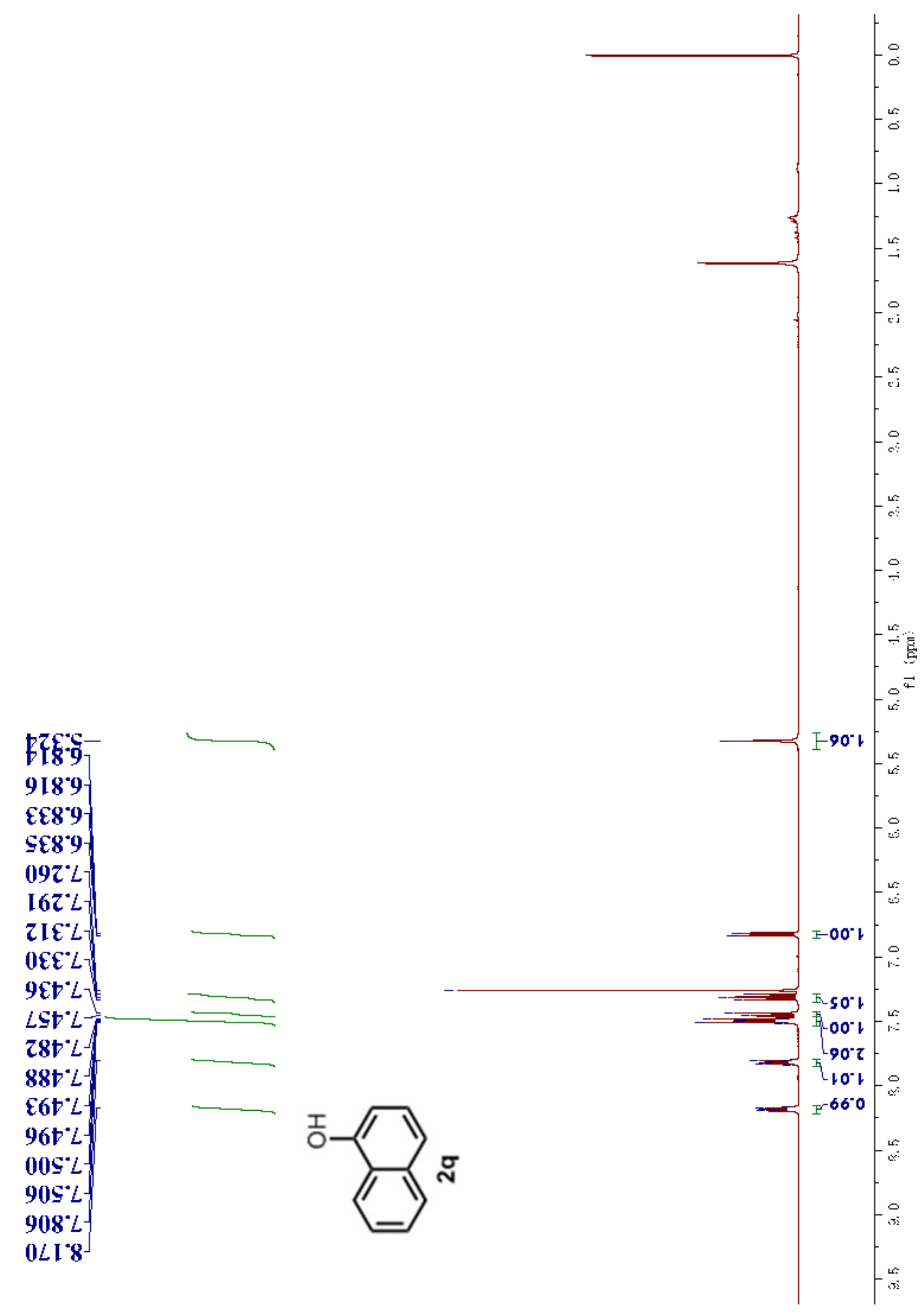



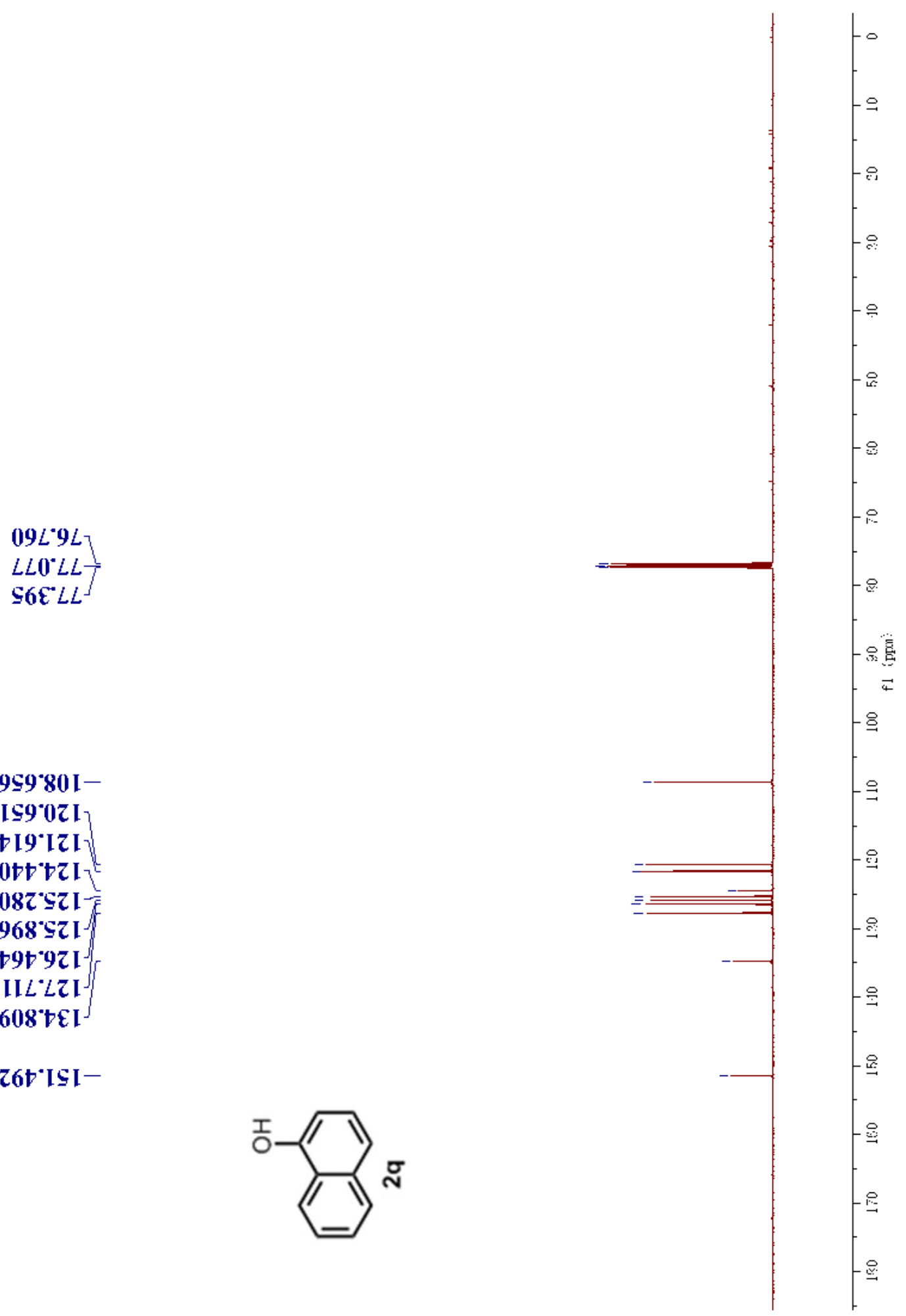


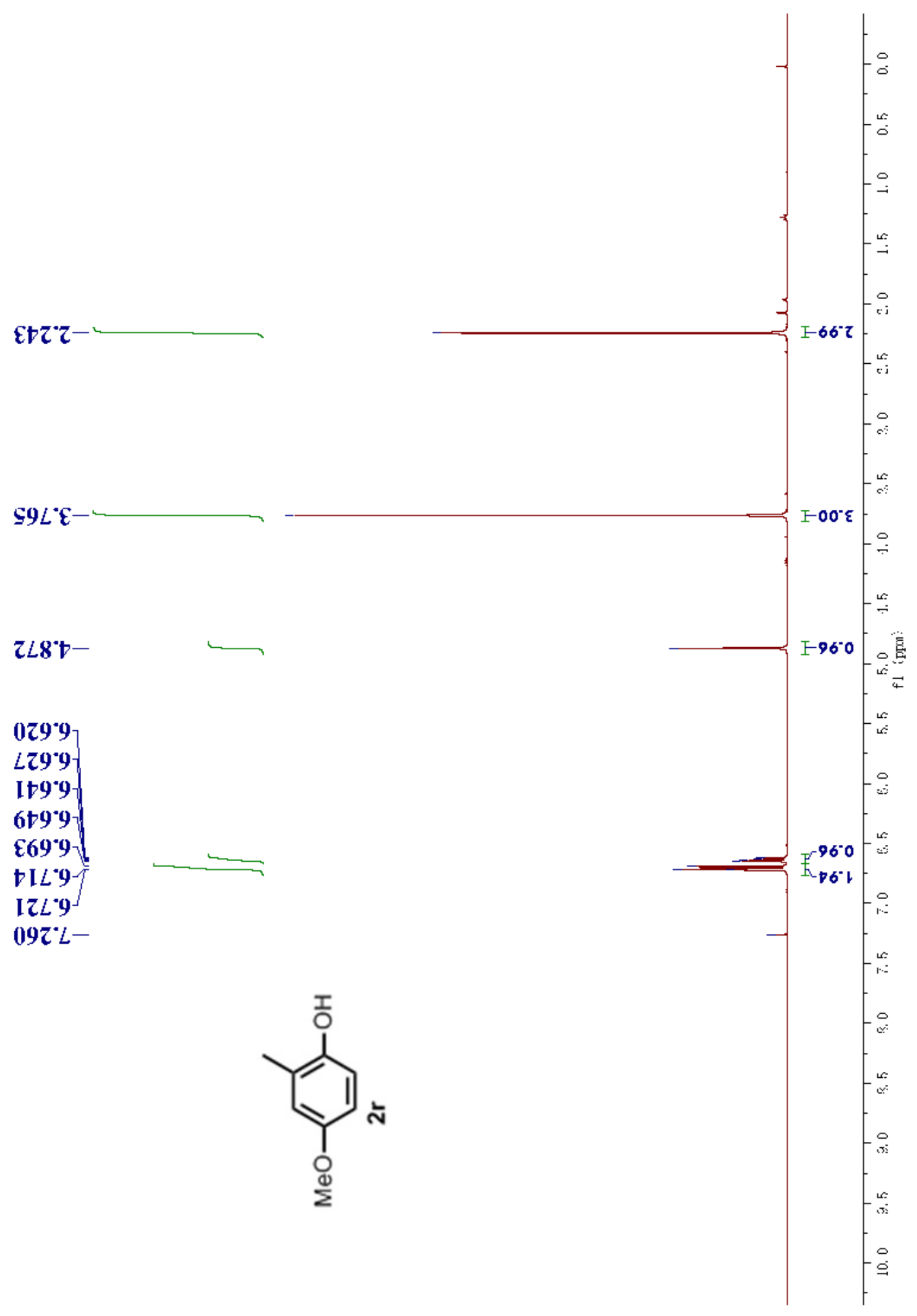


$9 z 7^{\circ} 9 \mathrm{I}-$

$806 \subseteq \varsigma-$

$\operatorname{cts} 8^{\circ} L$

$09 \mathrm{I}^{\circ} L L$

$8 L t^{\circ} L L$

t66 III

$089^{\circ}$ SI I $^{-}$

$\$ 8 L^{\circ} 9 \mathrm{II}^{\mathrm{J}}$

$\$ 6 I^{\circ} \subseteq I^{-}$

$\angle 96^{\circ} \angle t I-$

$9 \angle S^{\circ} \mathrm{ESI}-$

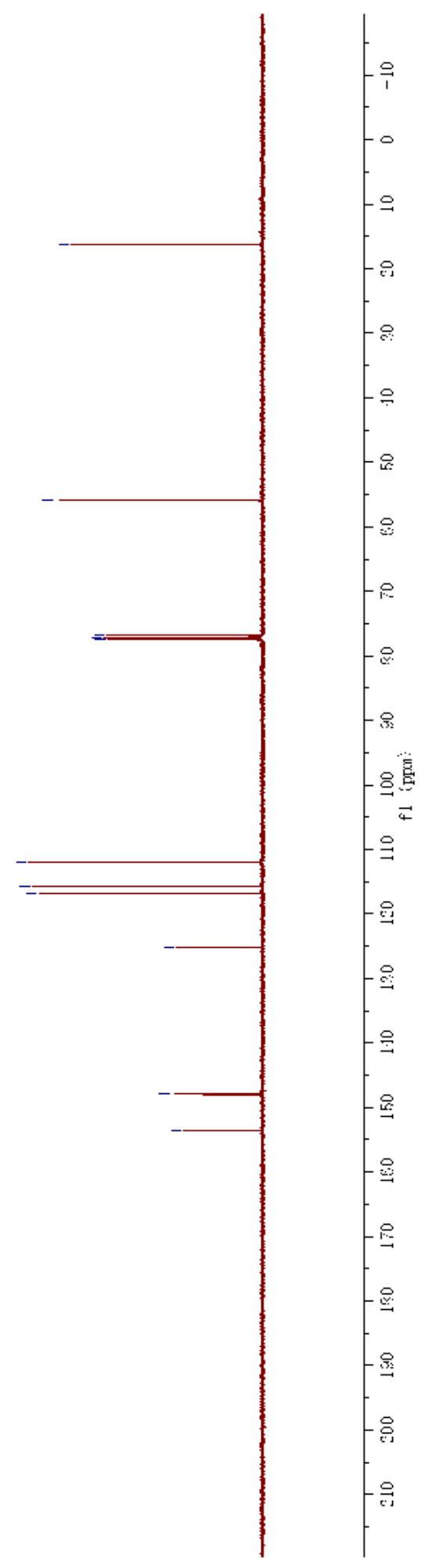




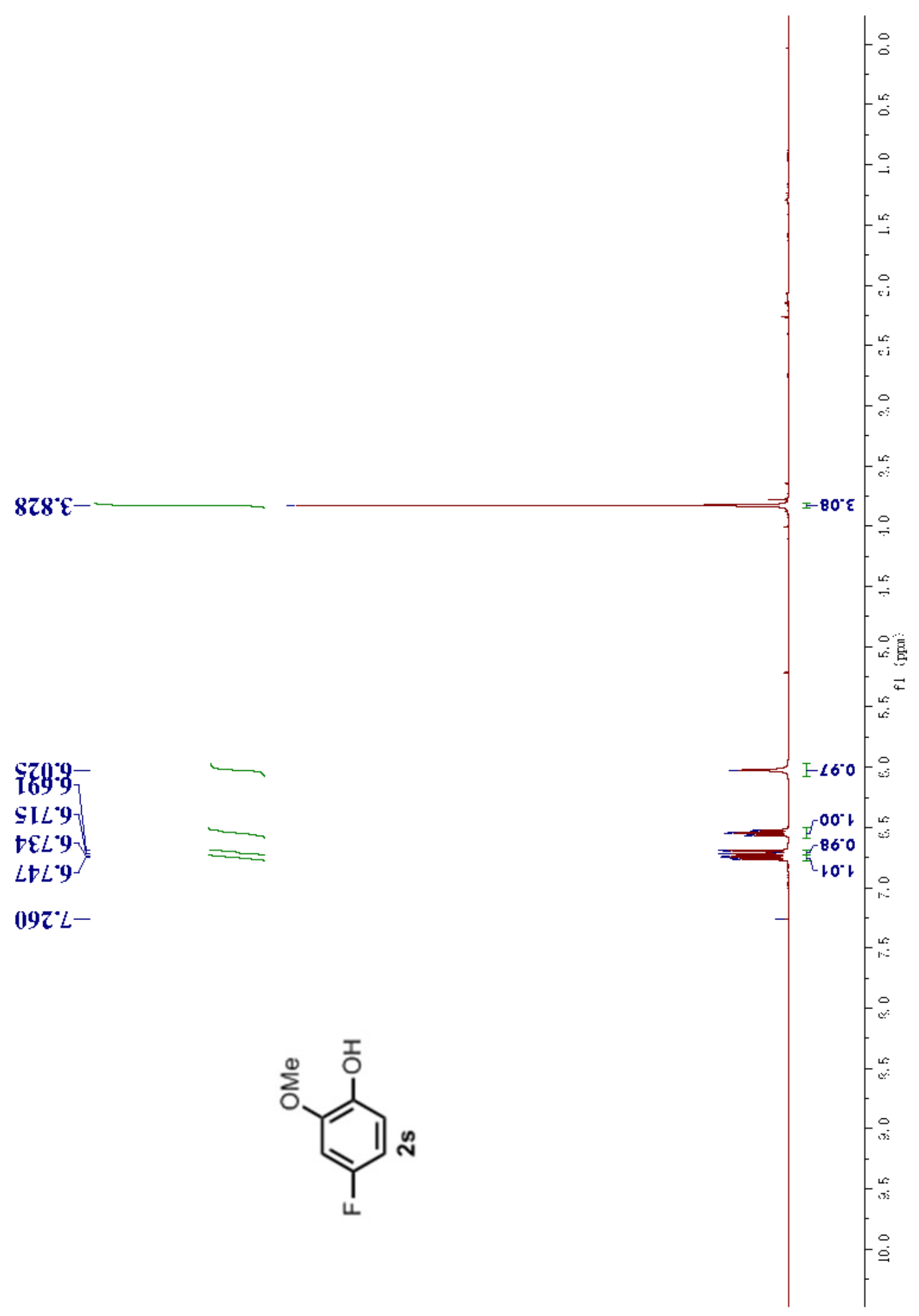


S6E9S-

$\mathbf{z}+8^{\circ} 9 L$

$09 \mathrm{I}^{\circ} L L$
$8 L 5^{\circ} L L$

E69 20 I

[96 $\mathrm{Z0}$ I]

I0S $\subseteq$ I

$0 \varepsilon L \subseteq 0{ }^{\circ}$

$870^{\circ}$ LII

$\angle$ I' III

šI $I^{\circ} \boldsymbol{t} \mathbf{I}$

ISI'Et I

Ett $9 t \mathrm{I}$

895.9t I

$78 \varepsilon^{\circ} 9 \mathrm{~S}$ I

$\angle \sqcup L^{\circ} 8 \mathrm{SI}^{-}$

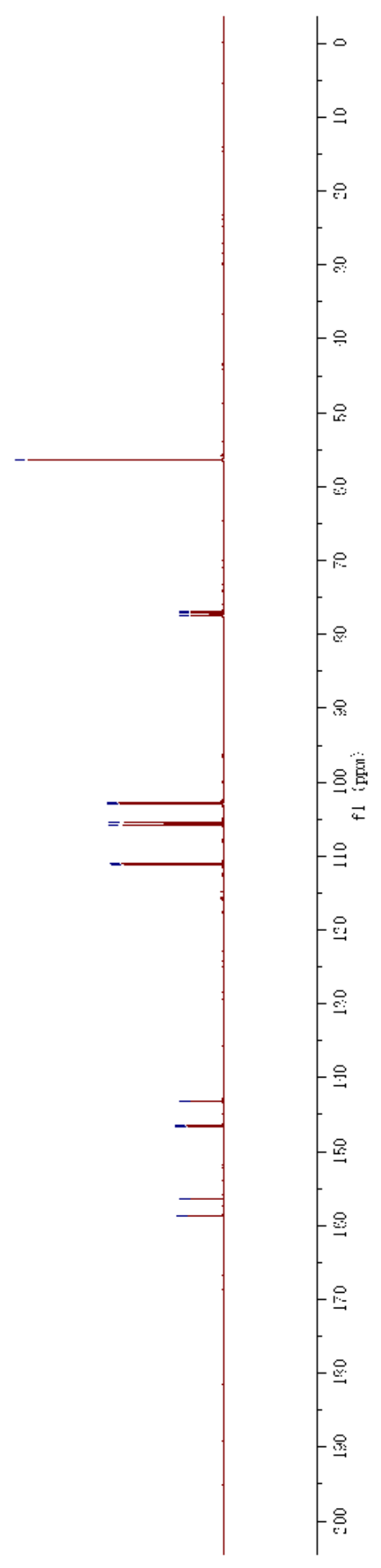


$00 \mathrm{z}-$

$6 \varepsilon I^{\circ} L$

$9 \mathrm{tI} / 2$

$\angle S I ' L$

$29 I^{\circ} \mathrm{L}$

$99 \mathrm{I}^{\circ} \mathrm{L}$

$L L I^{\circ} L$

$\left.\angle 8 \mathrm{I}^{\circ} \mathrm{L}\right]$

$\angle 6 \mathrm{I}^{\circ} \mathrm{L}$

$802: L$

I $20 \%$

$6208^{\circ}$

$\angle D I 8$

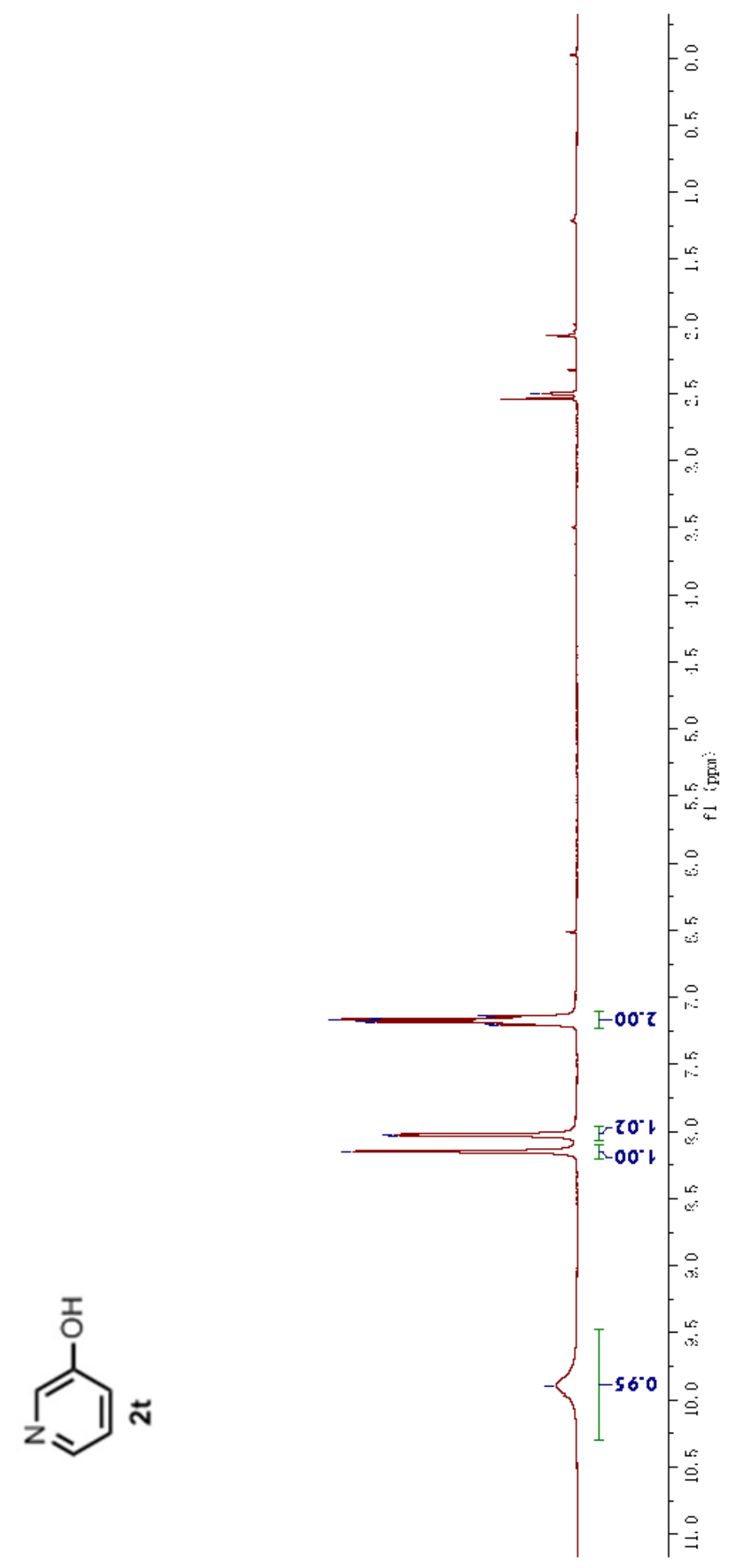




$$
\begin{aligned}
& \mathcal{E} 68^{\circ} 8 \varepsilon_{7} \\
& \text { zoI"6E } \\
& \text { II } \varepsilon * \varepsilon \\
& 0 z \subseteq 6 \varepsilon \\
& 6 z L^{\circ} 6 \varepsilon \\
& 8 \varepsilon 6^{\circ} 6 \varepsilon \\
& \text { 9t I. } 0 \mathrm{t} \\
& \text { IEt } \boldsymbol{t}^{\circ}
\end{aligned}
$$

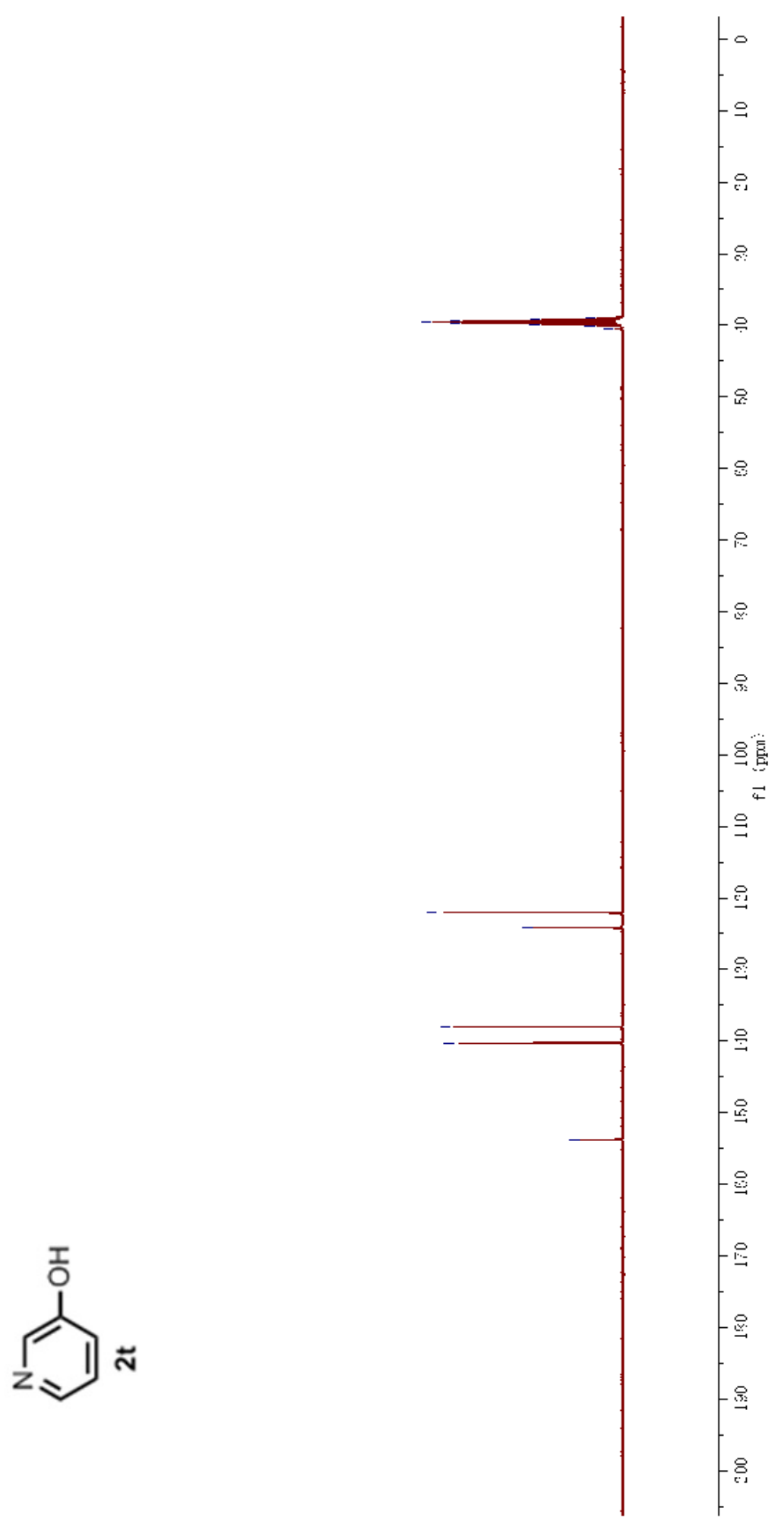

$\$ 80^{\circ} Z Z \mathrm{I}$

$\mathrm{I}^{\circ} \mathrm{t} t \mathrm{I}^{-}$

Z008EI

It $z^{\circ} 0 \mathrm{tI}^{-}$

$09 \angle \mathrm{ESI}-$ 
$00 \mathrm{~s} z$

$999^{\circ} 2$

$290^{\circ} \mathrm{L}$

$6 \angle 0^{\circ} L$

$S 0 \varepsilon^{*} L$

$s z \varepsilon^{*} L$

$8+\varepsilon^{*} L$

$99 E^{\circ} L$

$98 E^{\circ} L$

It I 8

$29 \mathrm{I}^{\circ}$

$900^{\circ} 6-$

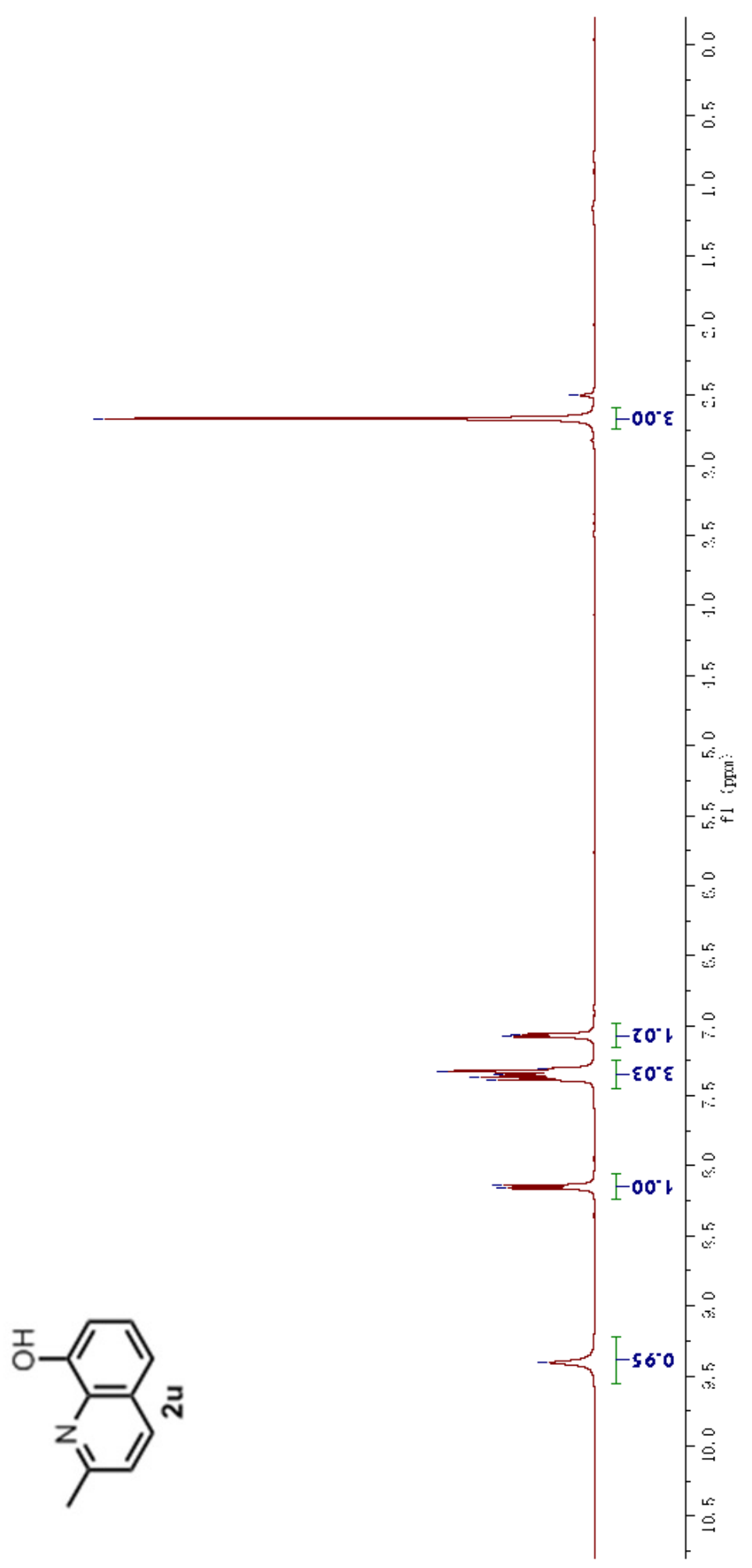




$$
\left.\begin{array}{l}
z \angle 9^{\circ} \downarrow z- \\
z 68^{\circ} 8 \varepsilon \\
z 0 I^{\circ} 6 \varepsilon \\
L I \varepsilon^{\circ} 6 \varepsilon- \\
0 z S^{\circ} 6 \varepsilon \\
8 z L L^{\circ} 6 \varepsilon \\
\angle E 6^{\circ} 6 \varepsilon \\
90 I^{\circ} 0 t^{\circ}
\end{array}\right]
$$
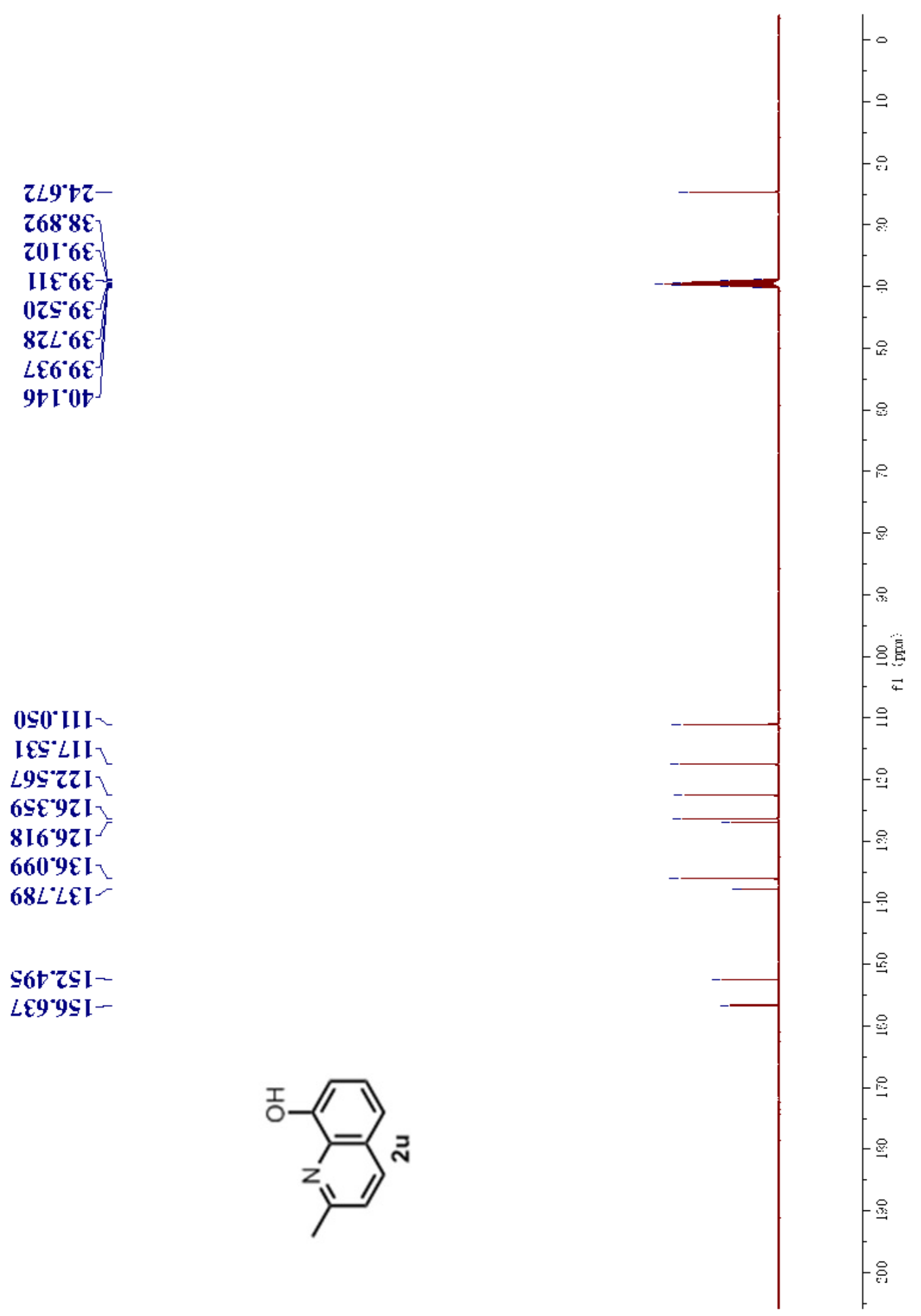


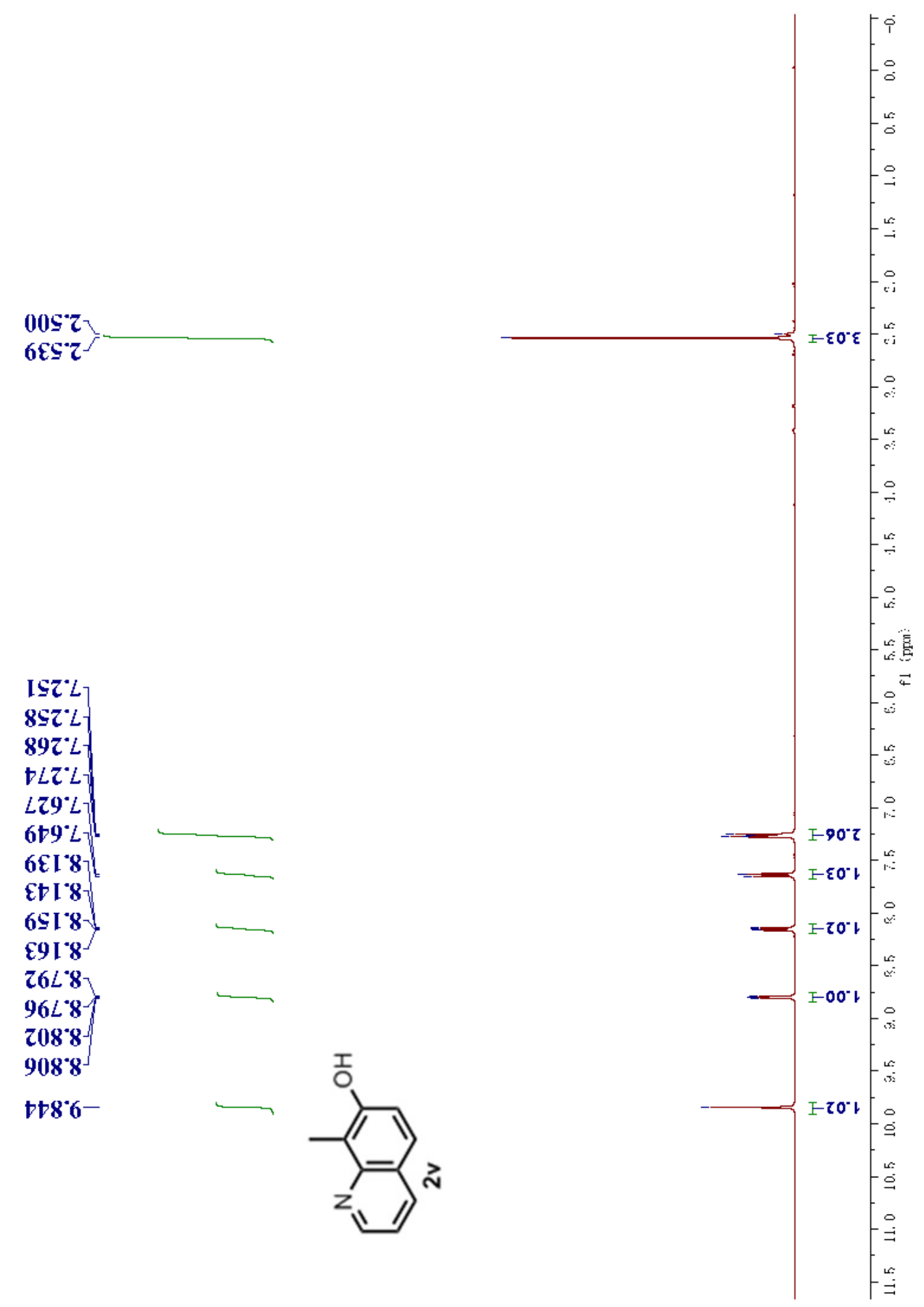




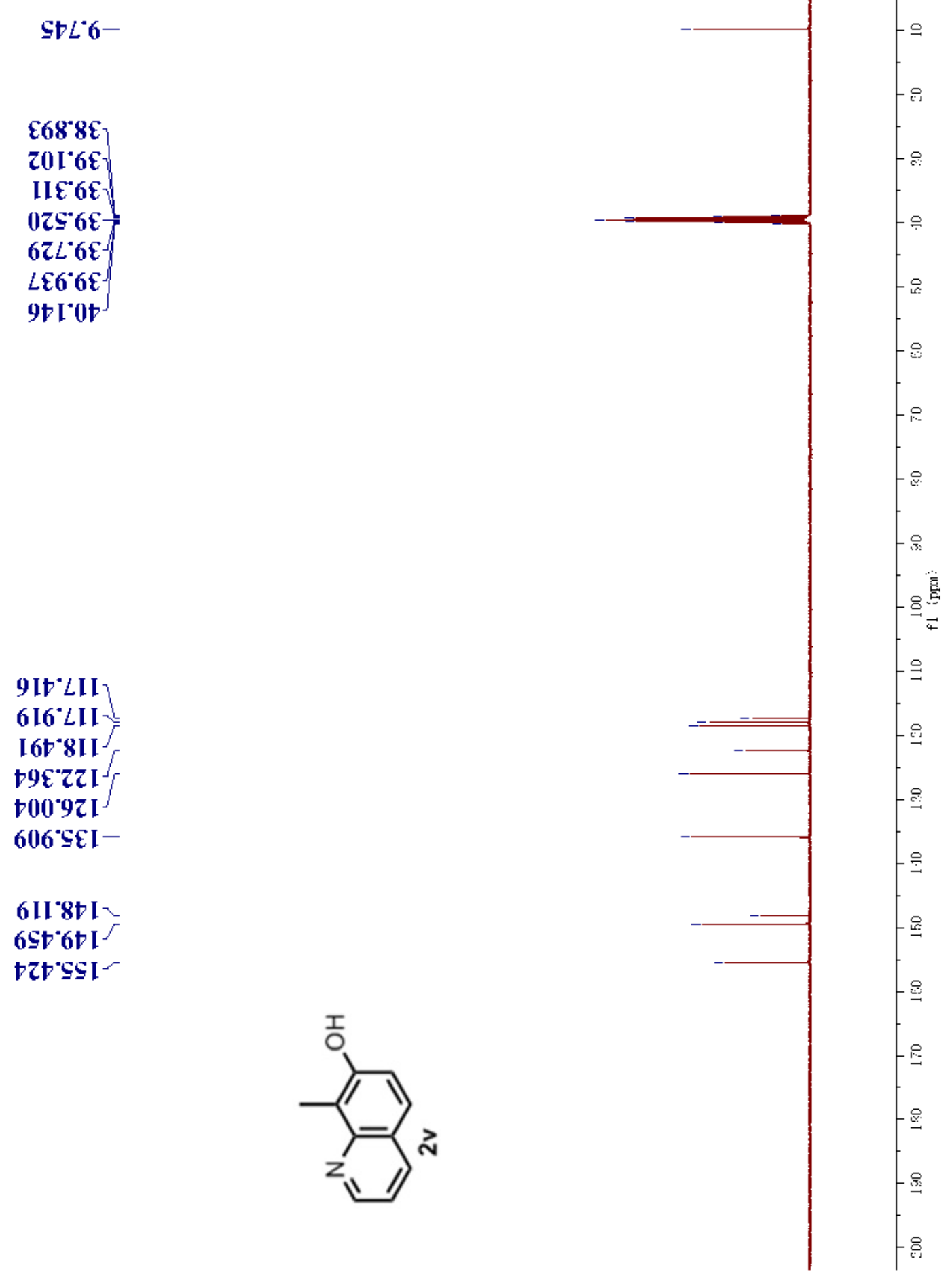




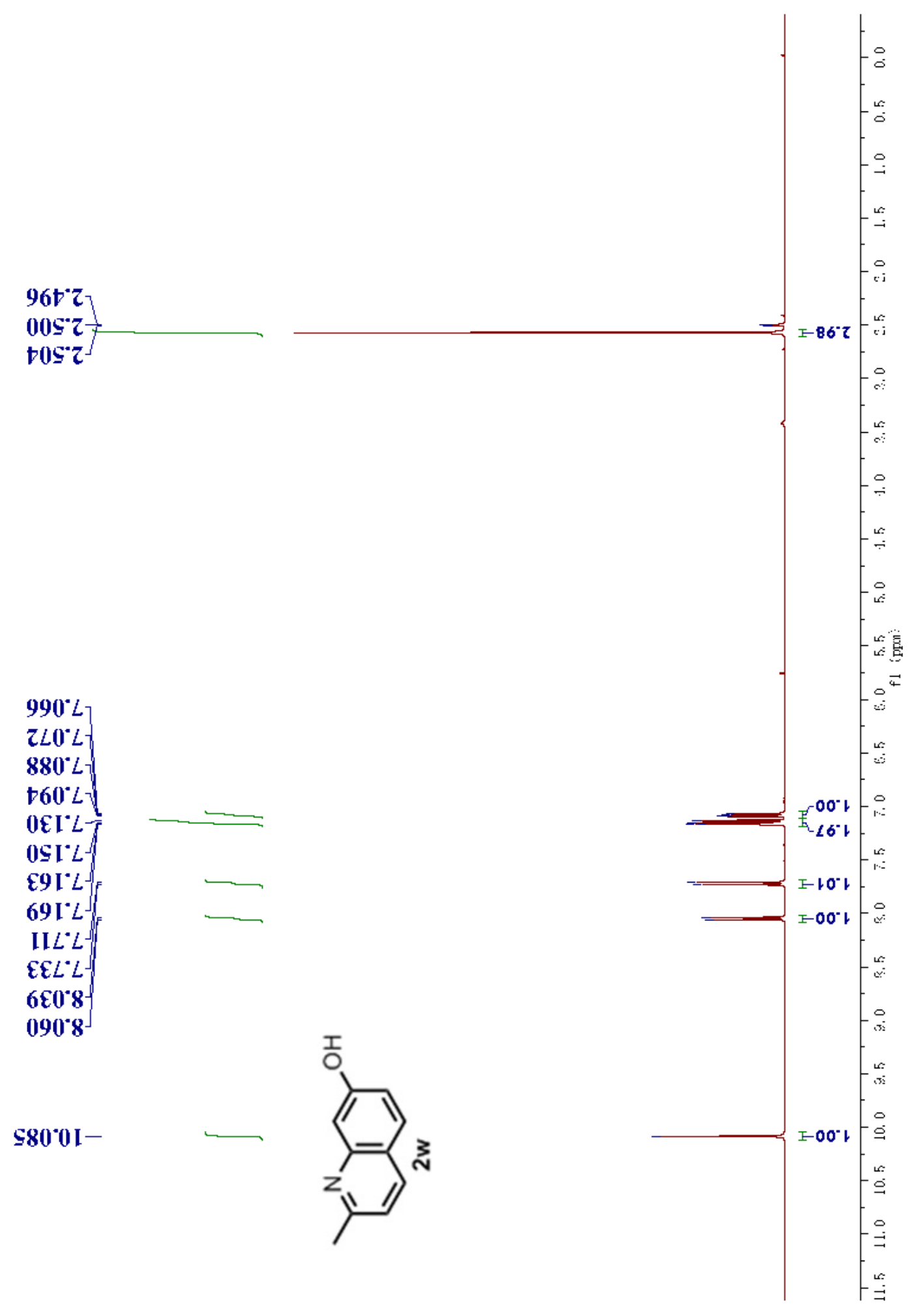




$$
\left.\begin{array}{l}
8 I 88^{\circ} z- \\
t 68^{\circ} 8 \varepsilon \\
z 0 I^{\circ} 6 \varepsilon \\
\text { II } \varepsilon^{\circ} 6 \varepsilon \\
0 z S^{\circ} 6 \varepsilon \\
6 z L 6 \varepsilon \\
L E \sigma^{\circ} 6 \varepsilon \\
90 I^{\circ} 0 t^{\circ}
\end{array}\right]
$$
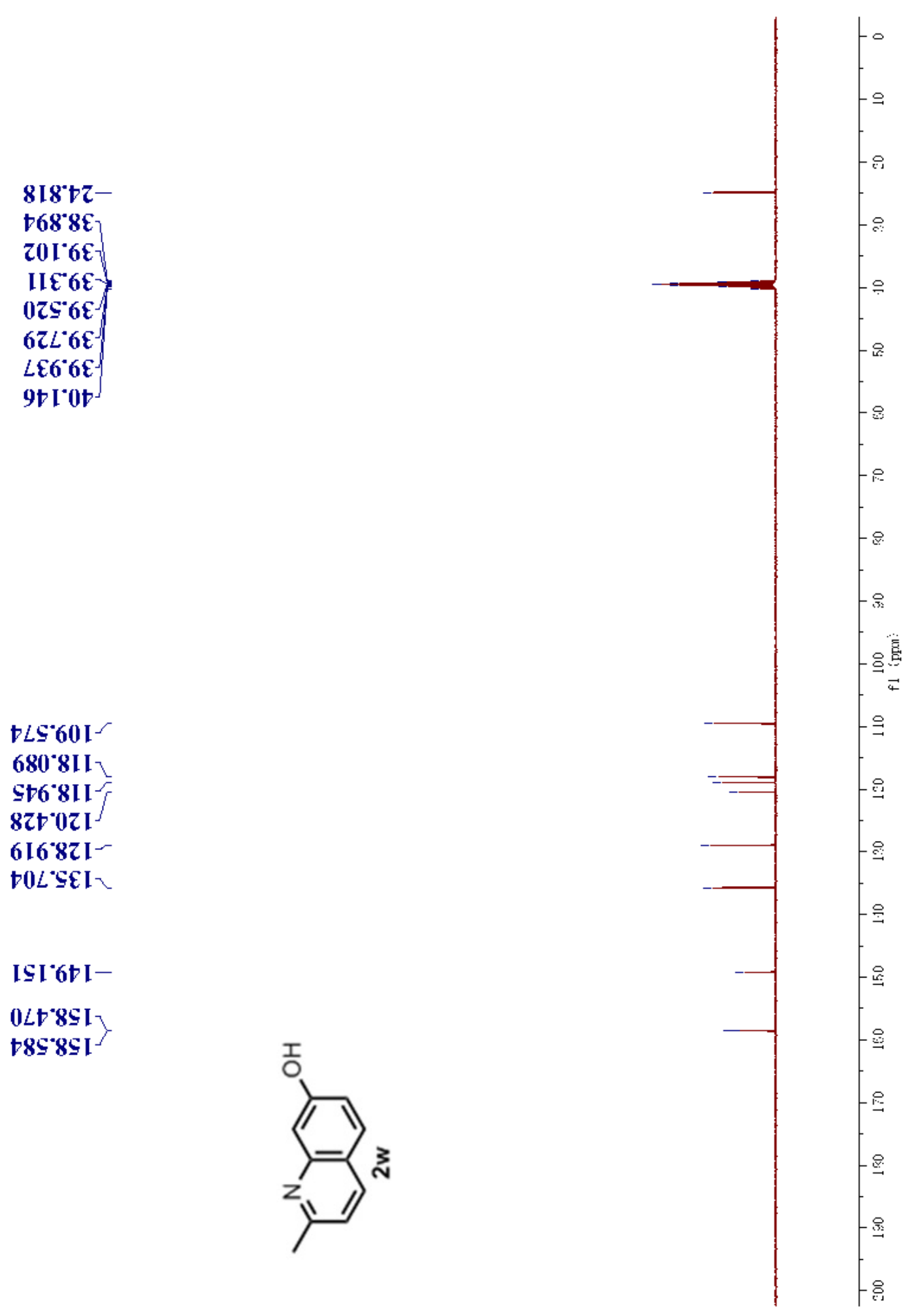


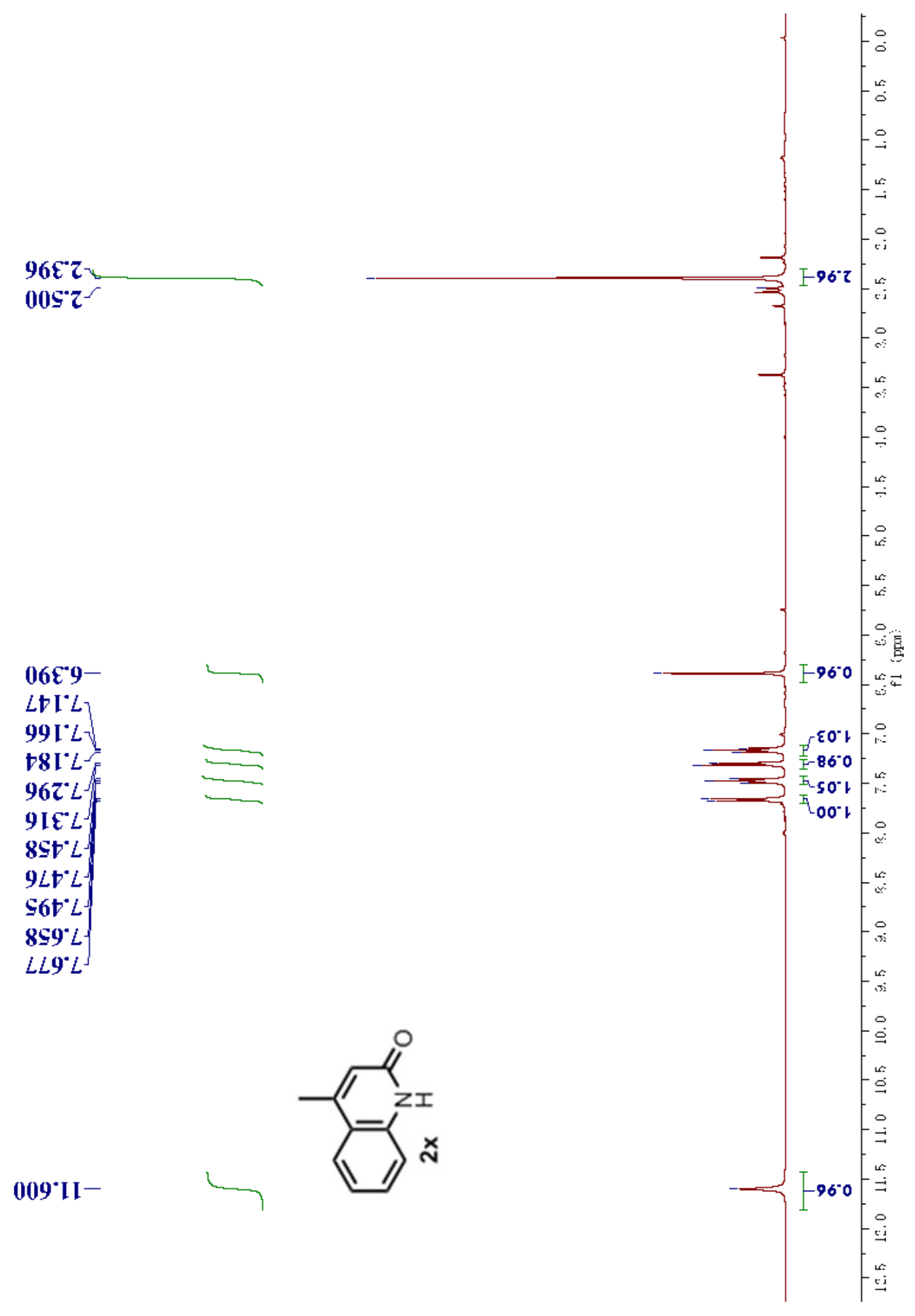


I $87^{\circ} 8 \mathrm{I}-$

† $68.8 \varepsilon$

$\mathcal{E} 0 \mathrm{I}^{\circ} 6 \mathcal{E}$

II $\varepsilon * 6 \varepsilon$

$0 z \Im 6$

$62 L^{\circ} 6 \varepsilon$

$\angle \mathcal{E} 6^{\circ} 6 \mathcal{E}$

$9 \mathrm{II}^{\circ} 0 \mathrm{t}^{\mathrm{s}}$

L0t'SI I -

E85:6 II-

Es80ZI

ZI9'IZI

$\angle L 9^{\circ} \pitchfork z \mathrm{I}$

$9 \varepsilon Z^{\circ} 0 \varepsilon \mathrm{I}^{-}$

$59{ }^{\circ} 8 \mathrm{I}$

$288^{\circ} \mathrm{L} \mathrm{I}^{-}$

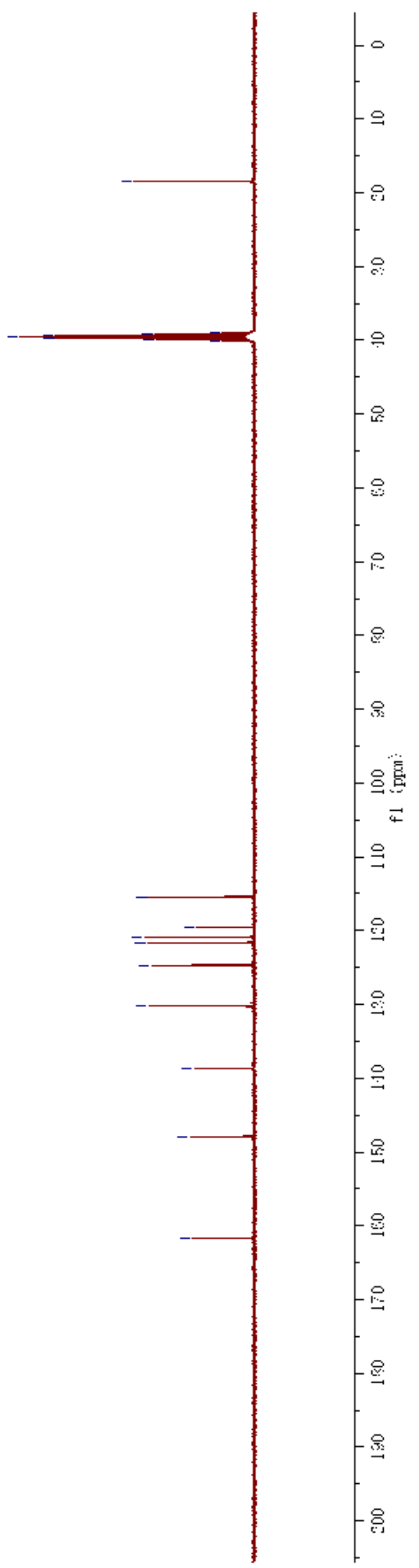


$\left.\begin{array}{l}\subseteq 6 t^{*} z \\ 00 S^{*} z \\ t 0 S^{\circ} z\end{array}\right]$

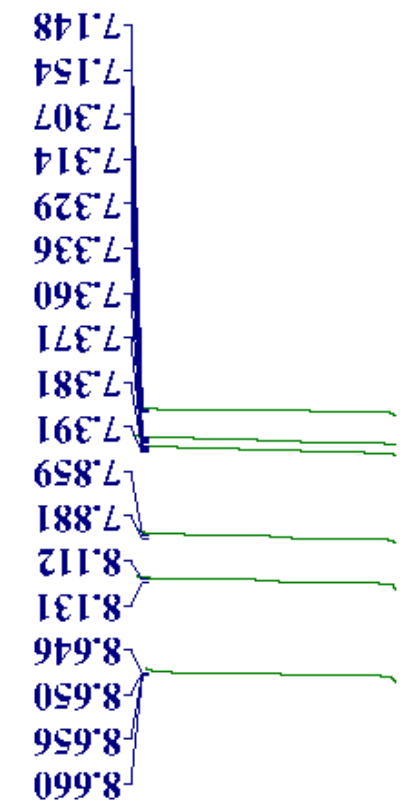

$9200^{\circ} \mathrm{I}$

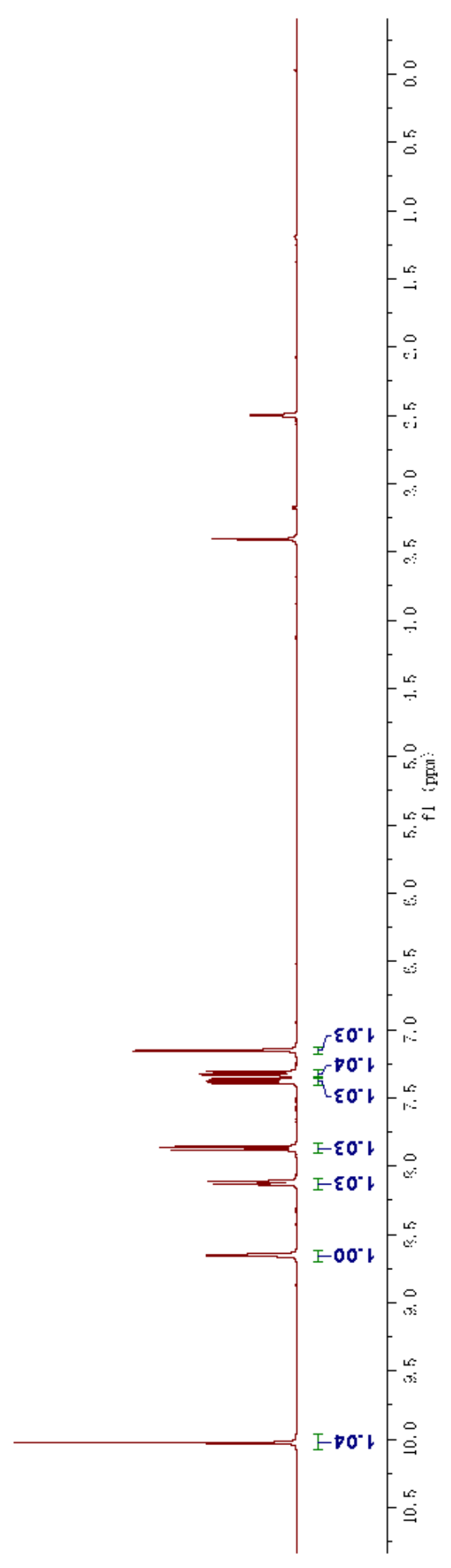




$$
\begin{aligned}
& \text { I } 68^{\circ} 8 \varepsilon_{7} \\
& \varepsilon 0 I^{\circ} 6 \varepsilon_{7} \\
& \text { zIE } 6 \varepsilon \\
& 0 z ङ 6 \varepsilon \\
& 6 z L 6 E \\
& \angle \mathcal{E} 6^{\circ} 6 \mathcal{E} \\
& \text { 9t I.0t }
\end{aligned}
$$

$\operatorname{ssc} 80 \mathrm{I}-$

\$8E IZI-

$566^{\circ} \mathrm{IZI}$

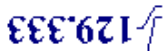

SIt $0 \varepsilon$ I

OEI'tE I

I $L 0^{\circ} \boldsymbol{\varepsilon} \boldsymbol{t}{ }^{\supset}$

$\angle$ II $^{\circ} L \mathbf{I}^{/}$

E6\% $9 s \mathrm{I}$

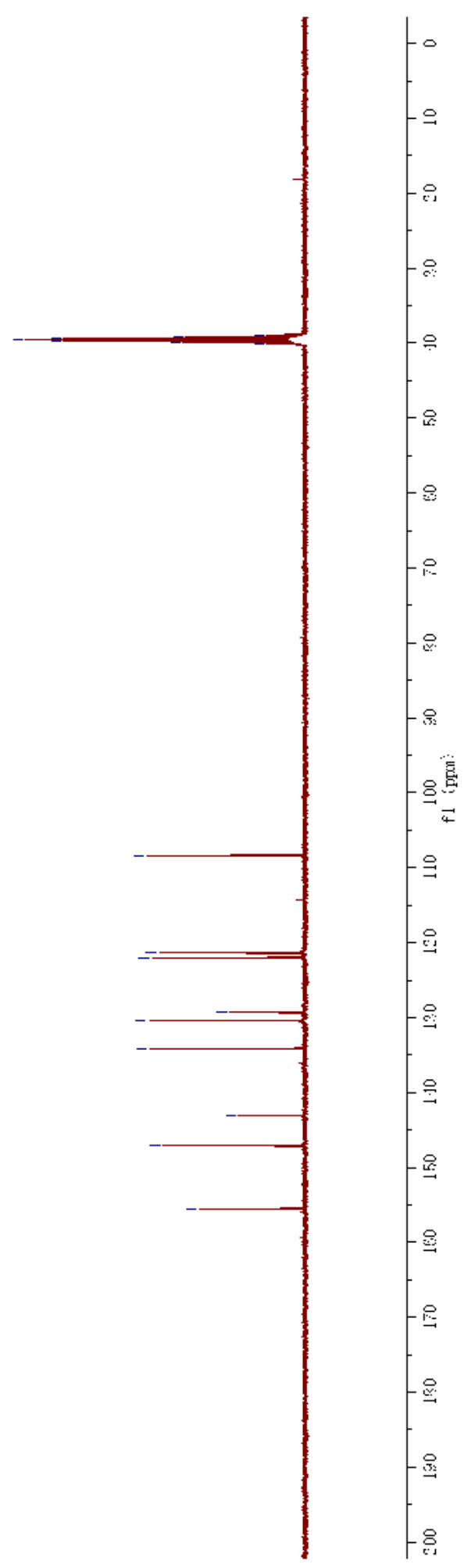



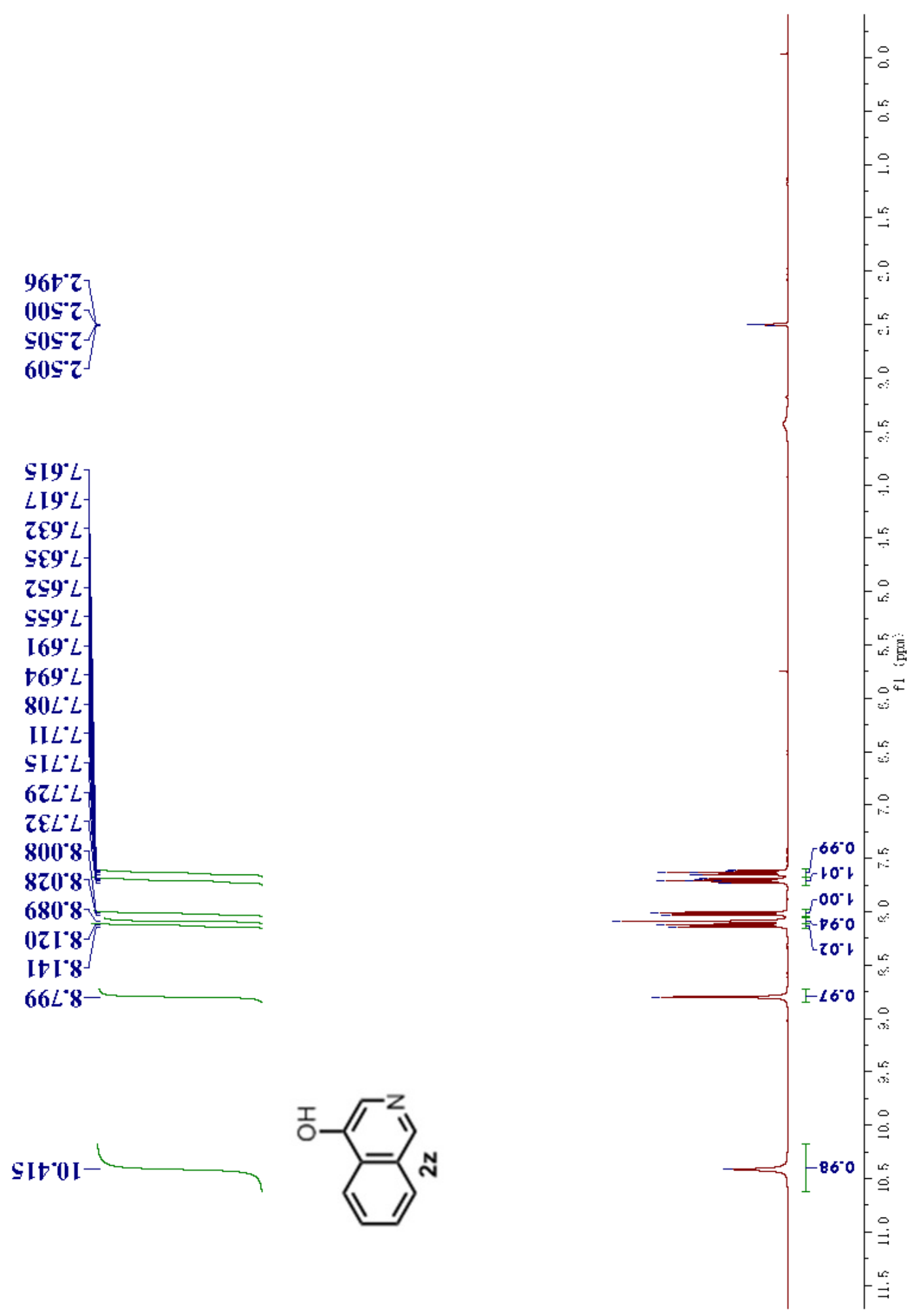


$$
\begin{aligned}
& \mathcal{E}^{6} 8^{\circ} 8 \varepsilon_{7} \\
& \text { I0I. } 6 \varepsilon_{7} \\
& \text { 0IE } 6 \varepsilon \\
& 0 z \Im 6 \varepsilon \\
& 6 z L 6 \varepsilon \\
& 6 \varepsilon 6^{\circ} 6 \mathcal{E} \\
& \angle I^{\circ} 0 t^{\prime}
\end{aligned}
$$

E8Z I I I

S68.9ZI

$\left.96 z^{\circ} \angle z\right]$

$9 \forall E^{\circ} L Z I$

$9 \mathrm{I0} 8 \mathrm{I}$

$0 S t^{\circ} 6 z \mathrm{I}$

$\varepsilon z L 6 z \mathrm{I}$

$0 \angle Z^{\circ} \varepsilon+\mathrm{I}-$

$88 L^{\circ} 8$ I $^{-}$

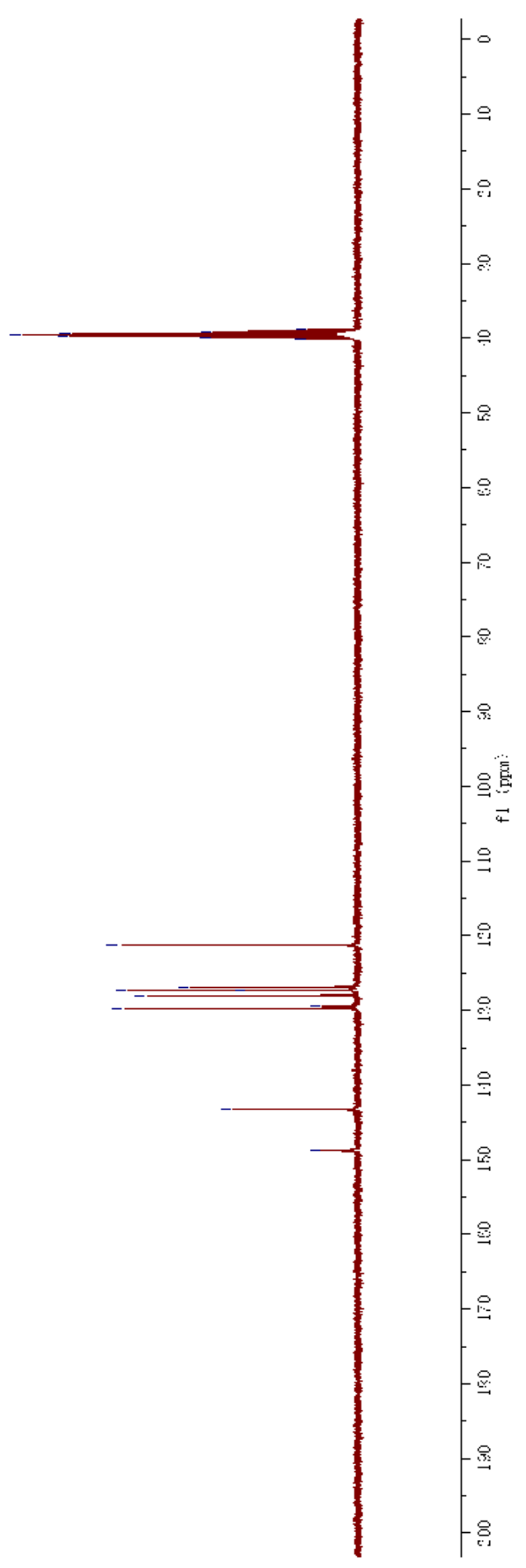


$96 t^{\circ} z$
$00{ }^{\circ} z$
t0S $z$

$69 \tau^{\circ} L$

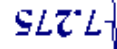

$96 \varepsilon L$

$\varepsilon 0 \sigma^{\circ} L$

$6 \mathrm{Lt}^{\circ} L$
$92 \mathrm{t}^{\circ} \mathrm{L}$

$026^{\circ} \mathrm{L}$

$276^{\circ} L^{\circ}$

$889^{\circ} 8$

$\angle 89^{\circ} 8$

I $L L \cdot 8$

$9 L L 8$
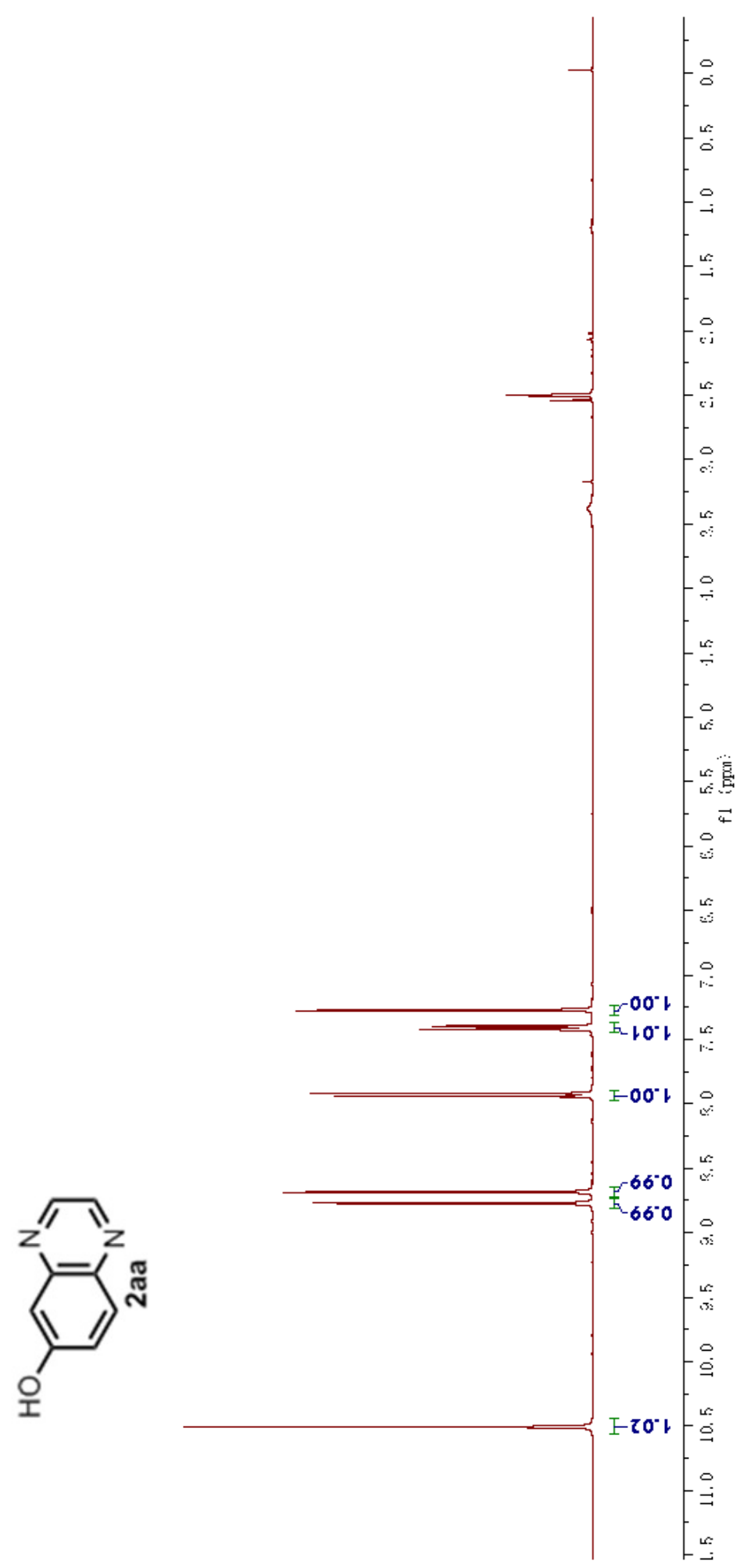


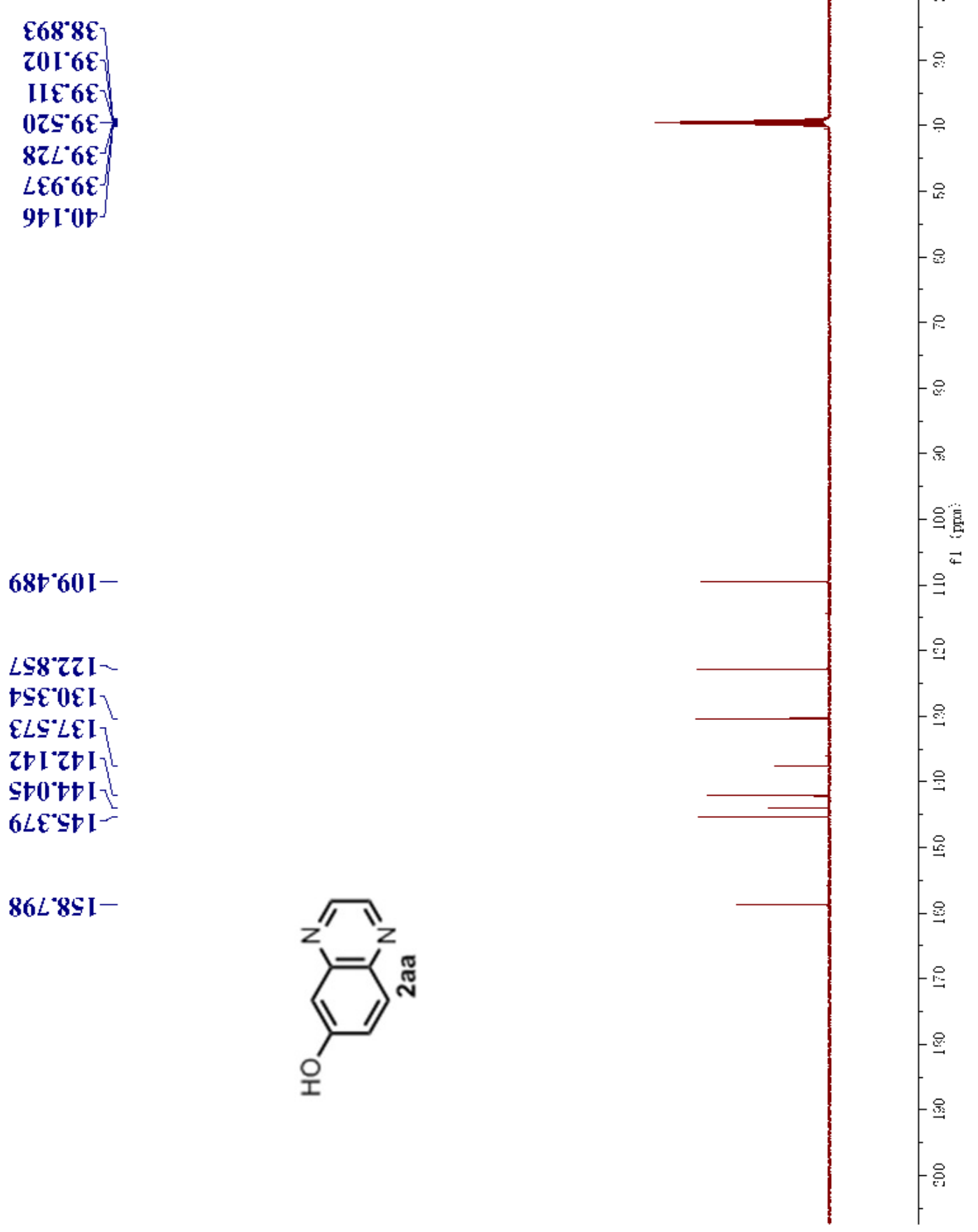




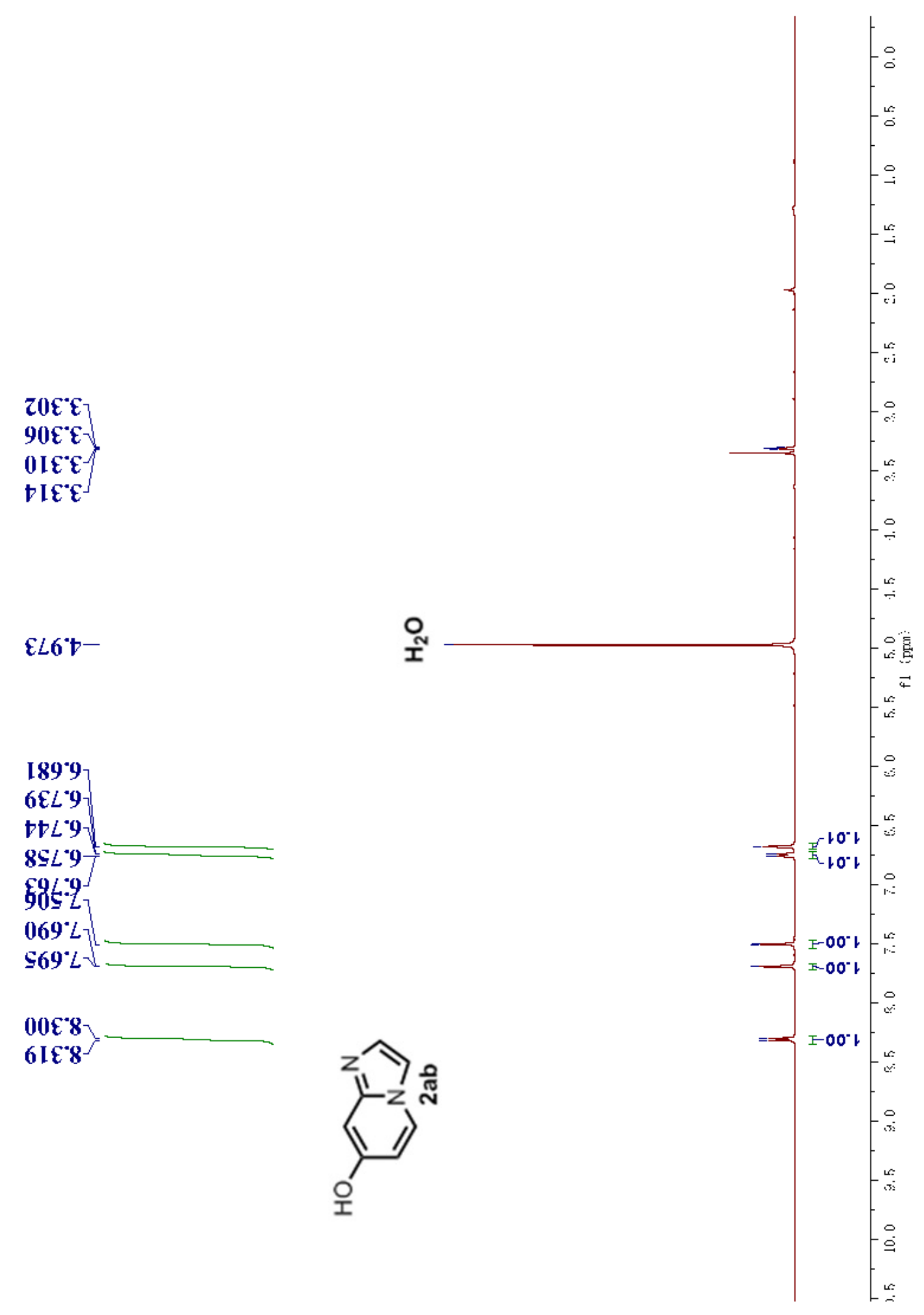




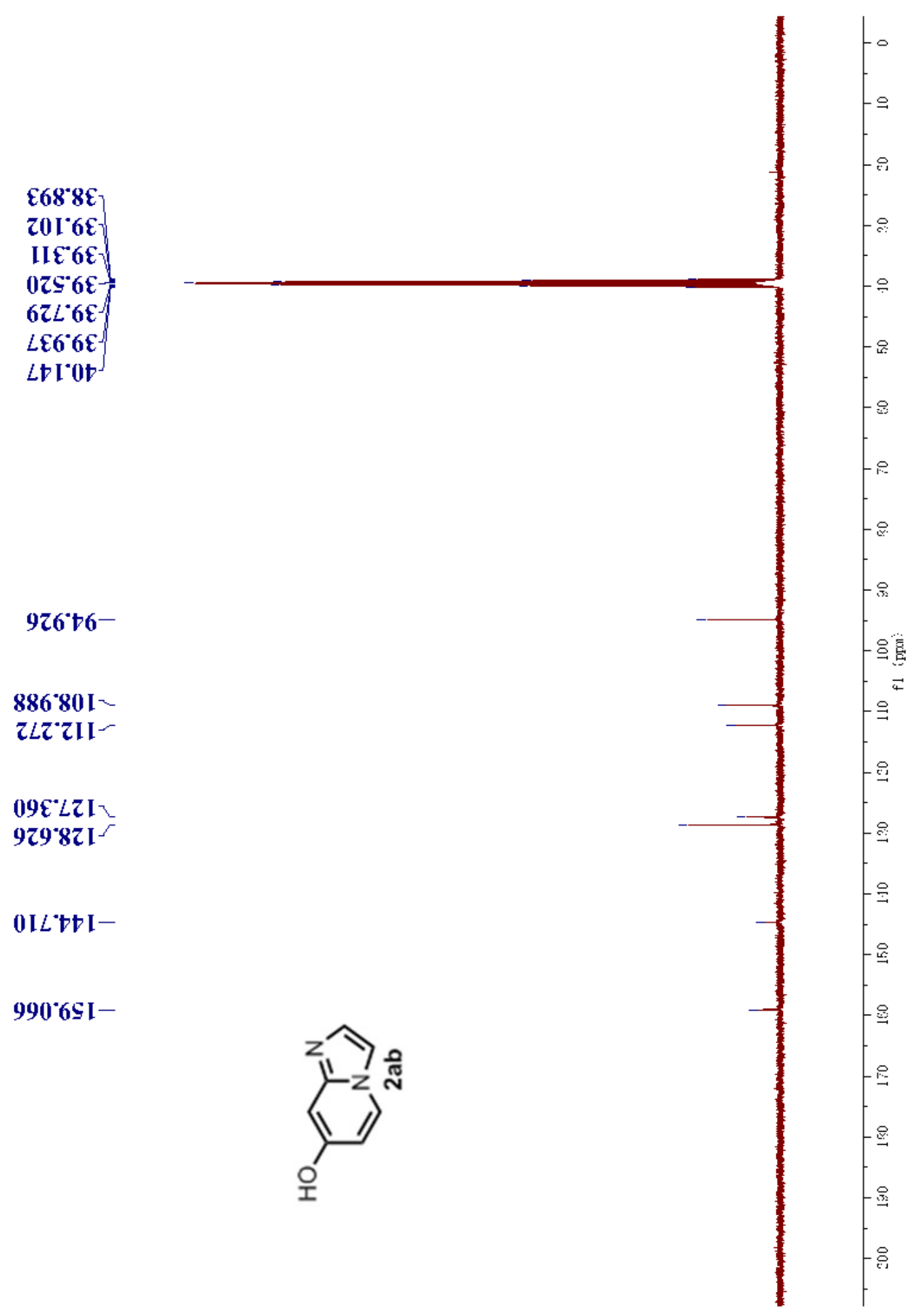


$\angle S \mathcal{E} z$

$09 \varepsilon z$

$\varepsilon 8 z^{\circ} \varsigma-$

$2 z 6^{\circ} 9$

$826^{\circ} 9$

$5060^{\circ}$

$0 \subseteq 6^{\circ} 9$

$\angle 80^{\circ} \angle$

$\operatorname{tE} I^{\circ} L$

$0 t I^{\circ} L$

$09 z^{\prime} L$

$9 \angle 9^{\circ} \mathrm{L}$

$\angle 69^{\circ} \mathrm{L}$

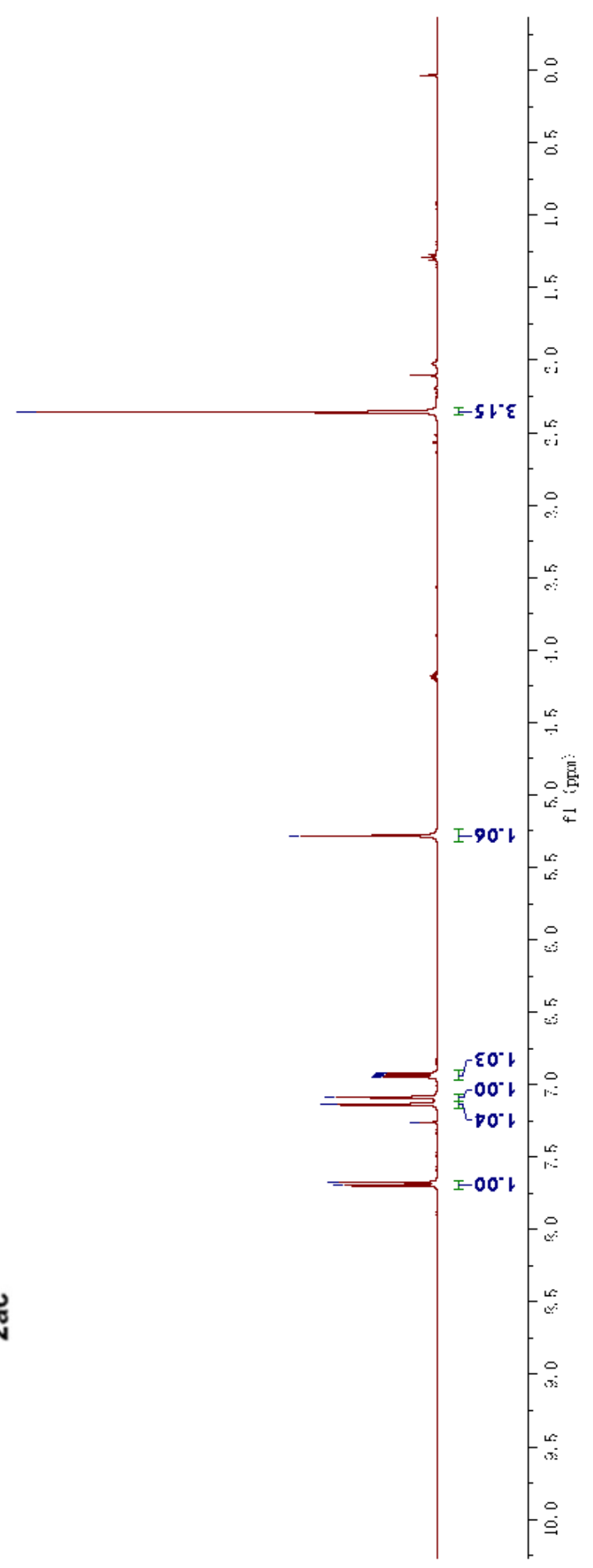


$086^{\circ} \varepsilon I^{-}$

$z+8^{\circ} 9 L$

$09 \mathrm{I}^{\circ} L L-$

$8 L T^{\circ} L L^{\prime}$

$99 \mathrm{I}^{\circ} \mathrm{L0I} \mathrm{I}^{\mathrm{S}}$

$8 \mathrm{I}^{\circ} \mathrm{tI}$

$6 z \tau \varepsilon z \mathrm{I}$

$\varepsilon \mathrm{L} L^{\prime} \varepsilon \mathrm{I}=$

9S9 $I \varepsilon I \backslash$

$\varepsilon+8 \cdot z \varepsilon I-$

$\angle t I^{\circ} I t I \backslash$

I86 $2 \mathrm{SI}-$

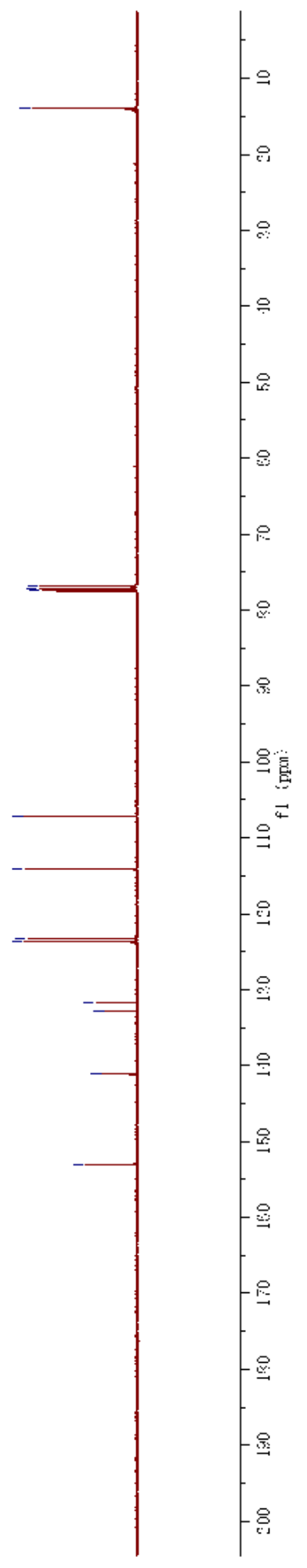




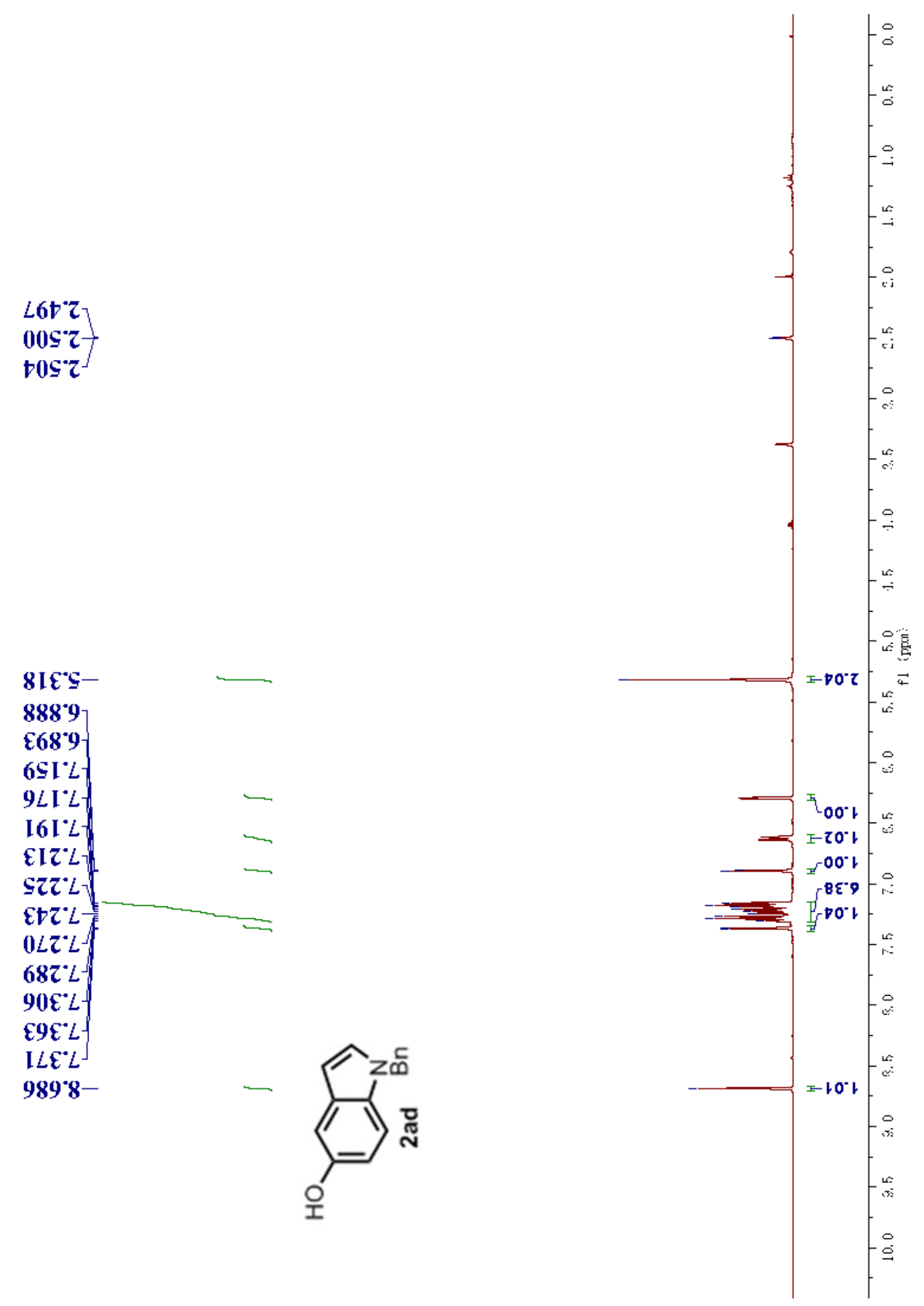




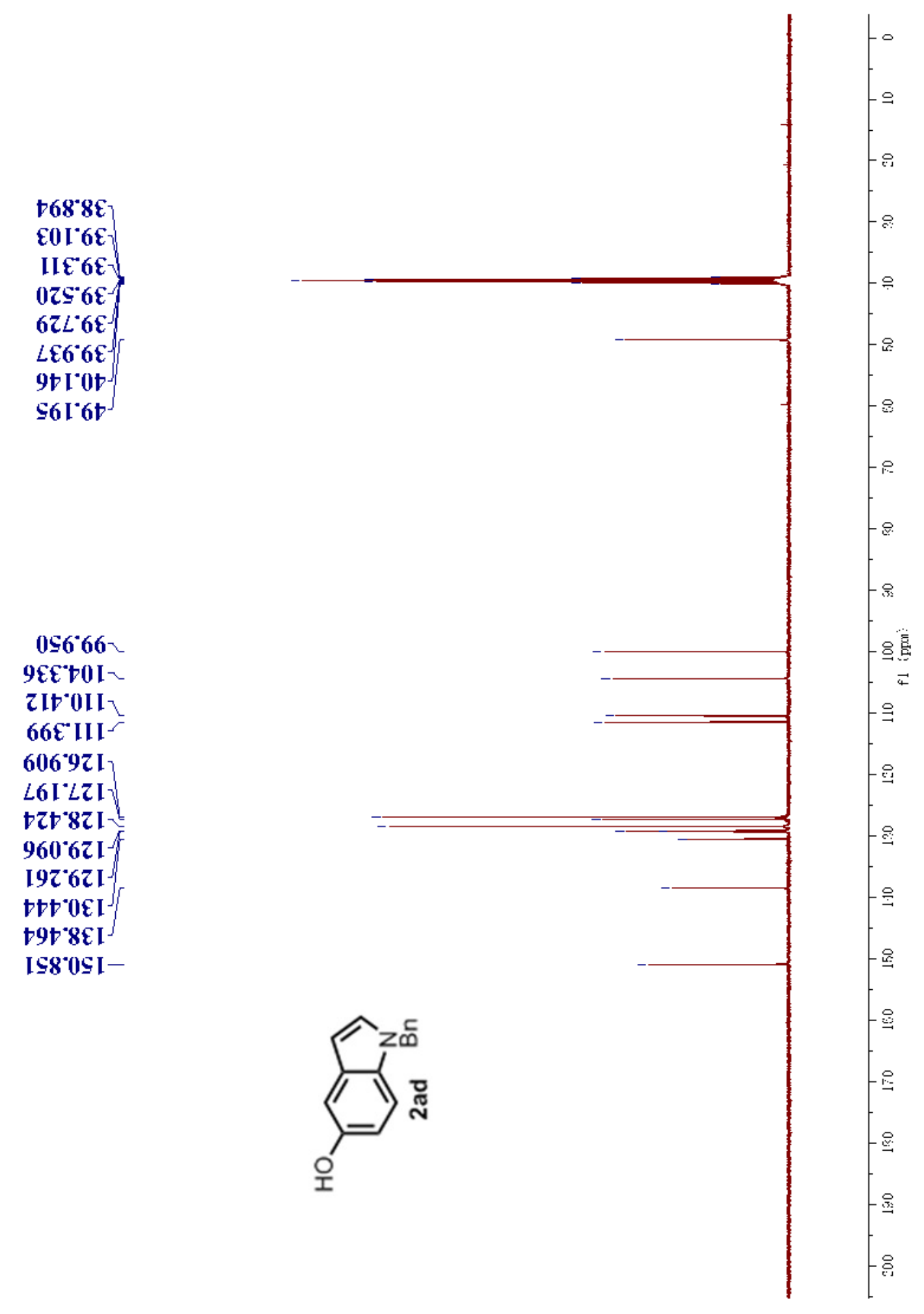




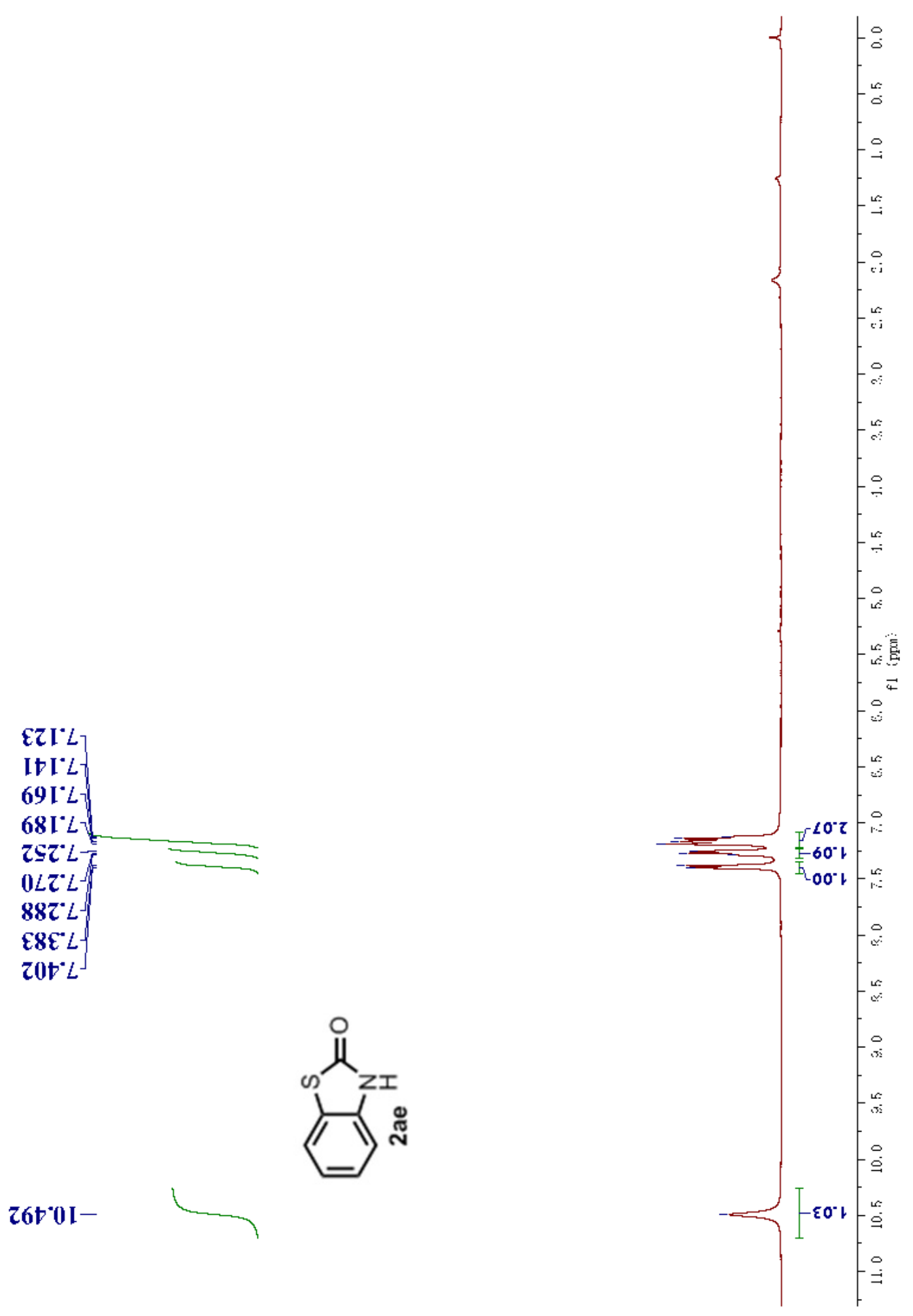




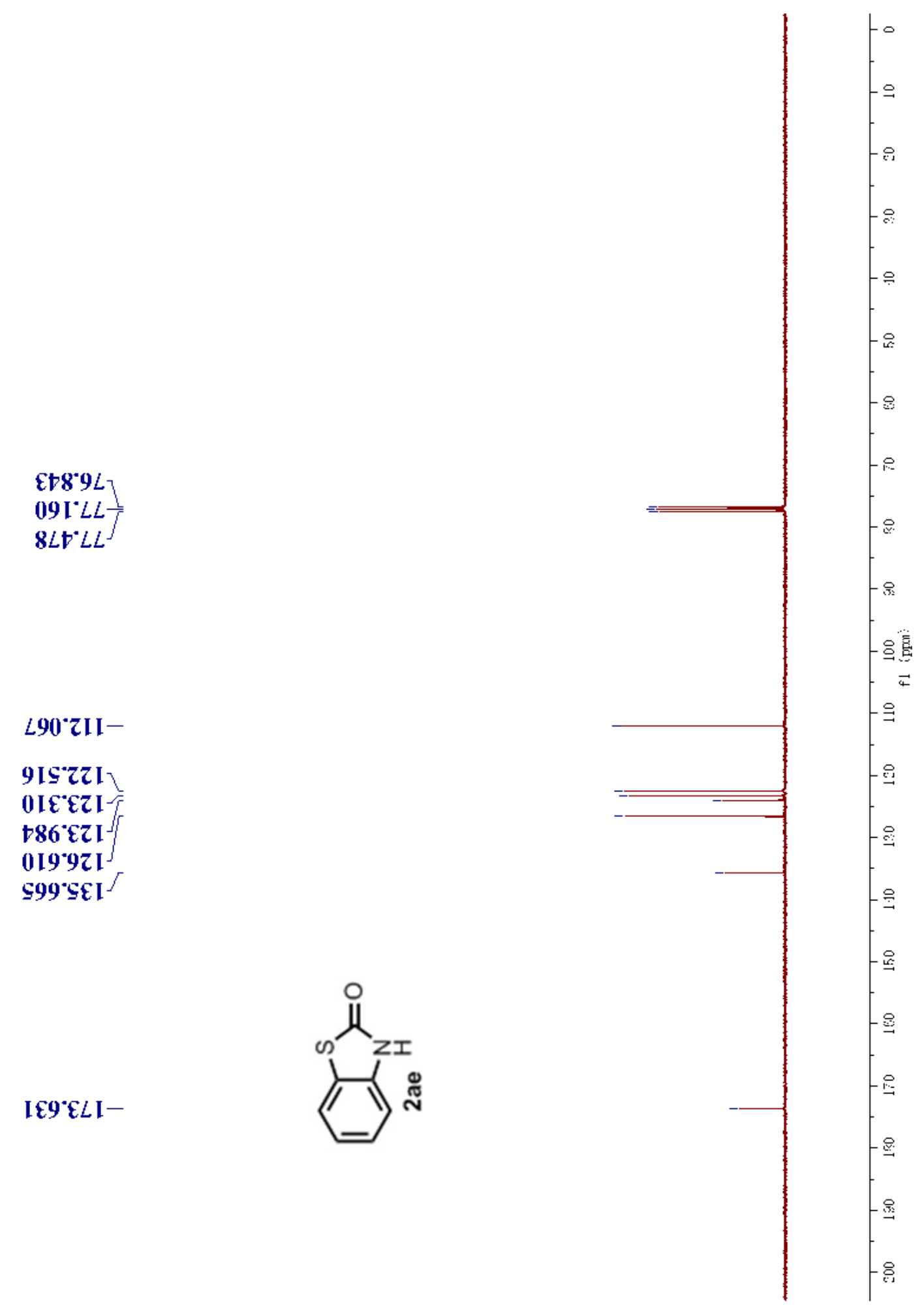




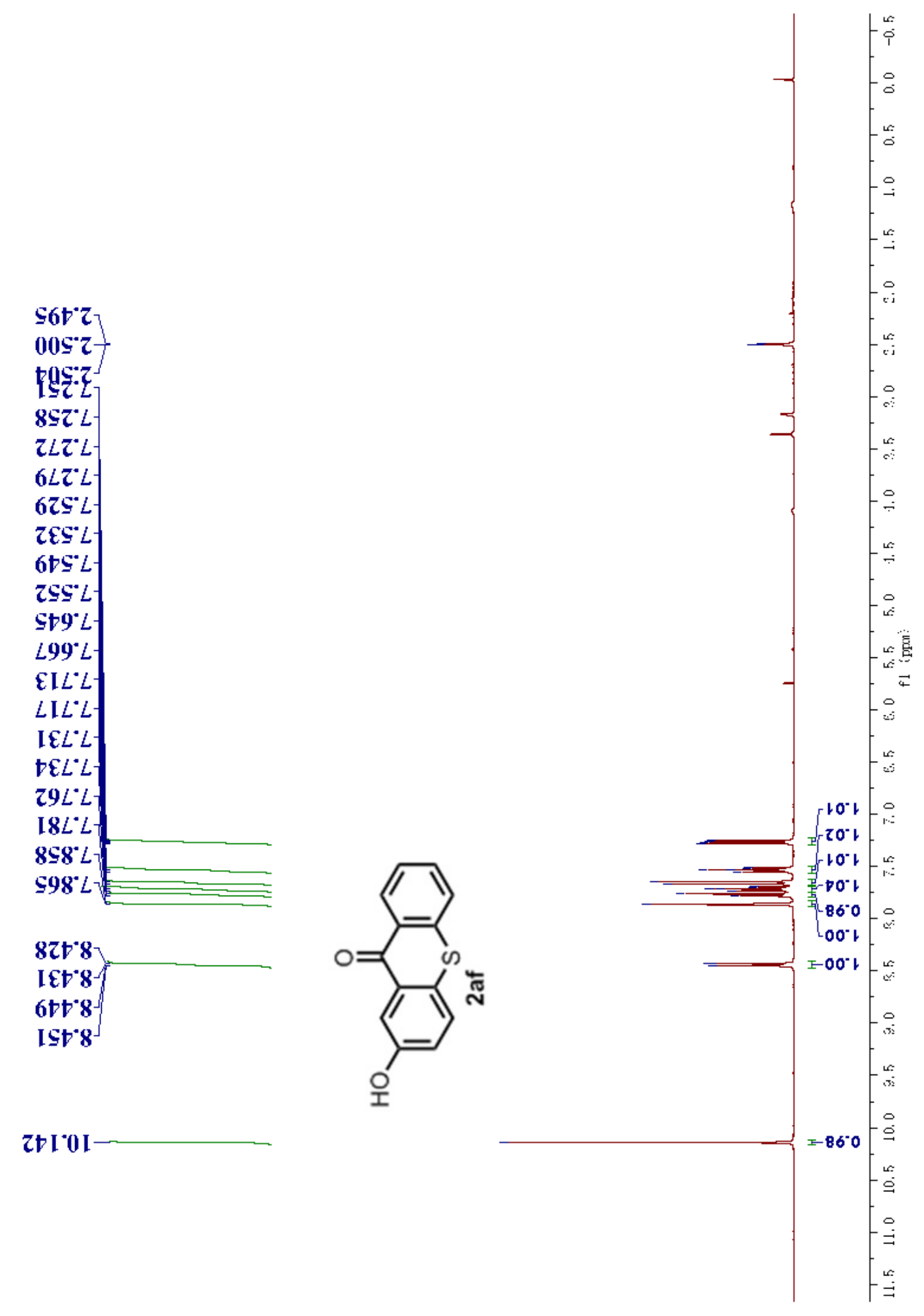




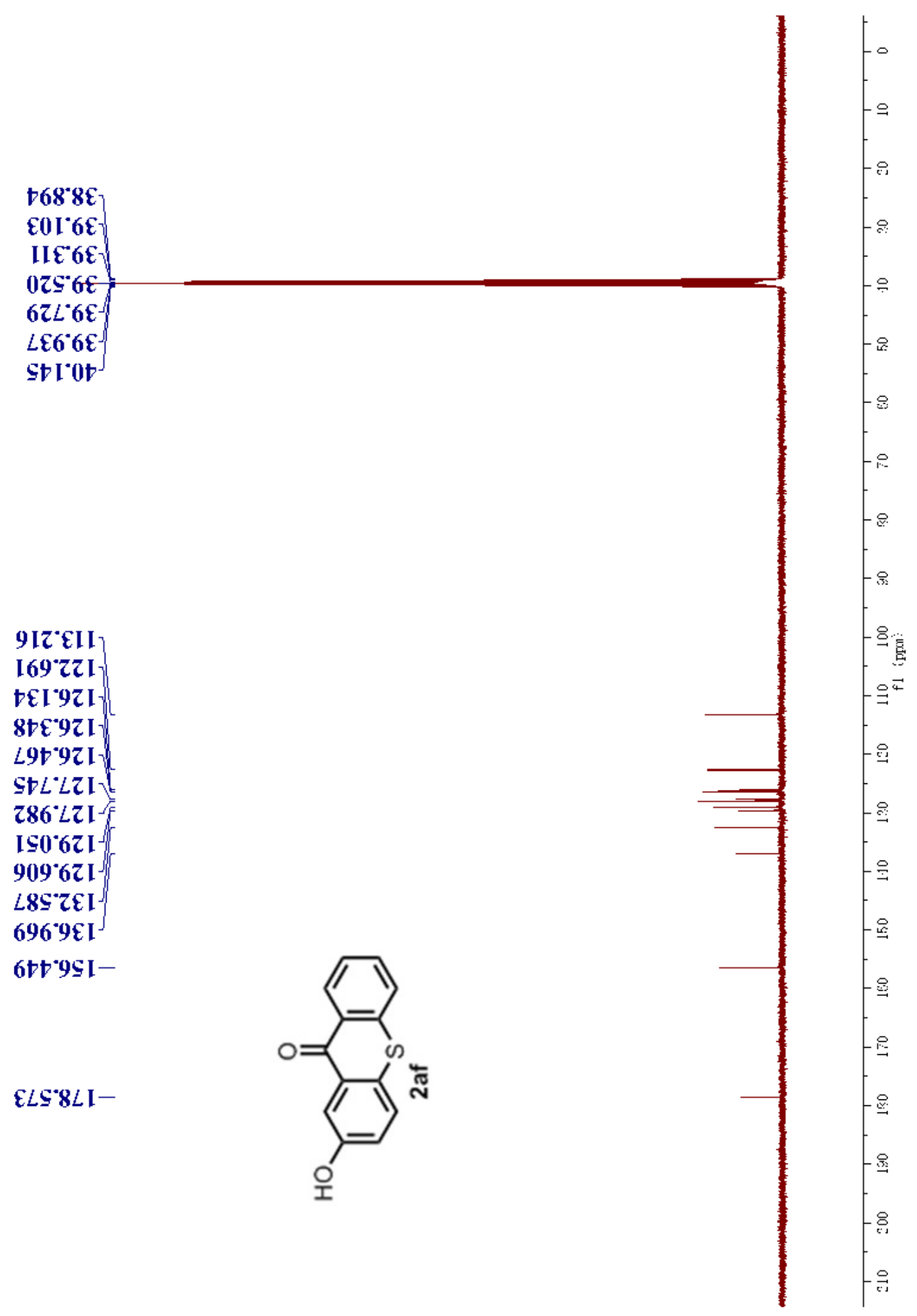




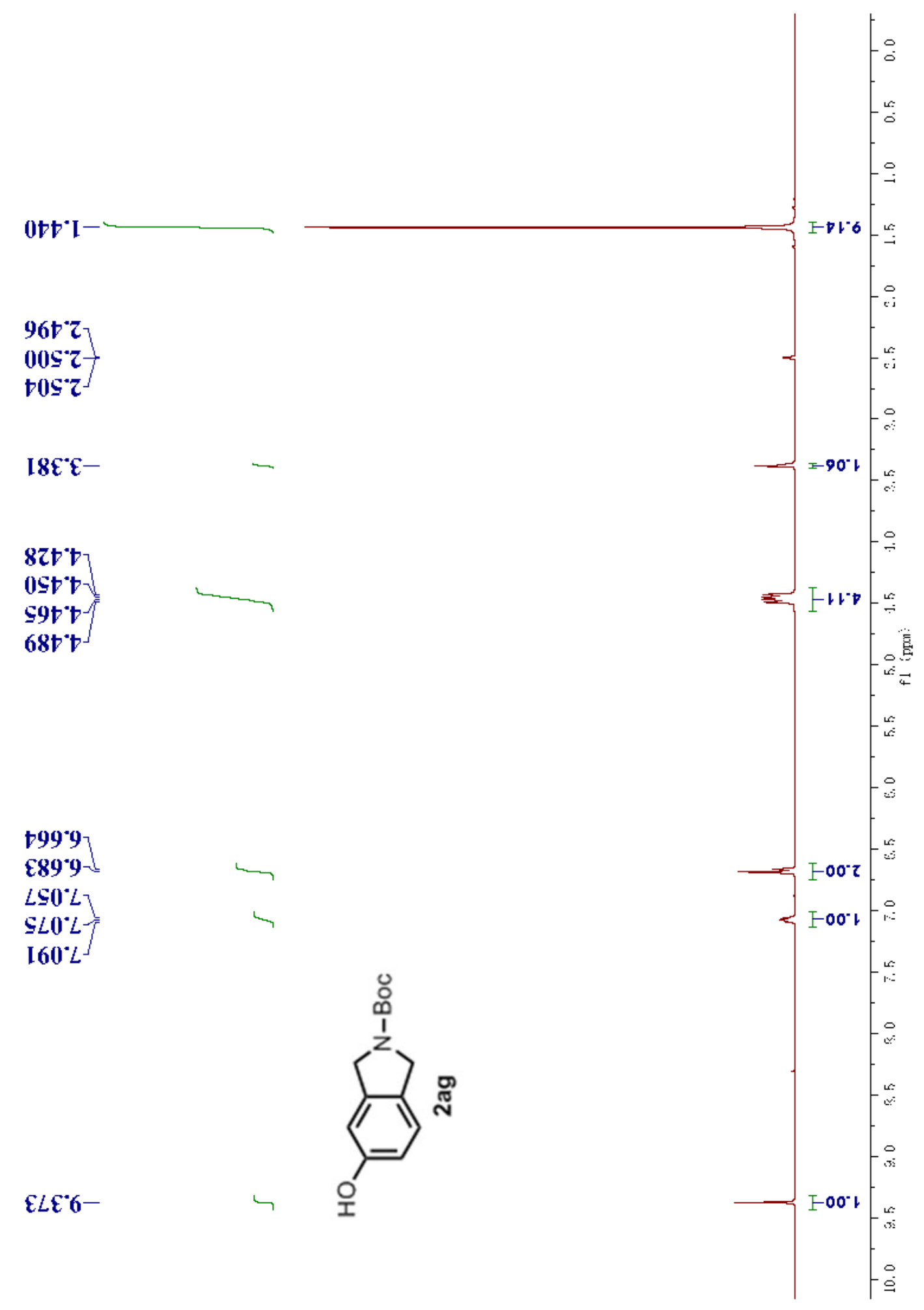




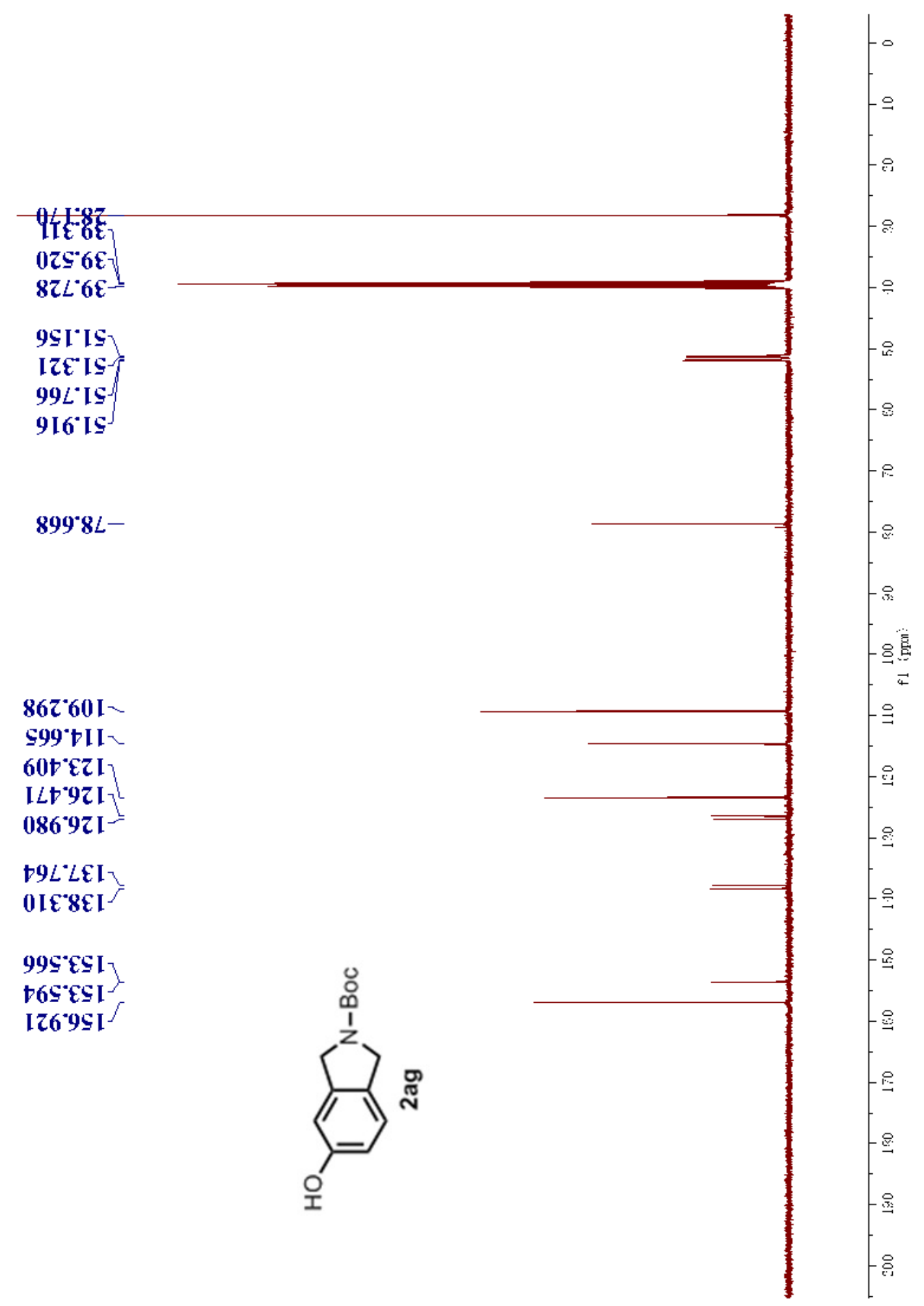


$00 \mathrm{~s} z-$

$085^{\circ} L$

EIS"L

$\varepsilon L L^{\circ} L$

I06 $L-7$

$5 \mathrm{~L} 6 \mathrm{~L}^{\circ}$

\$I9'8-

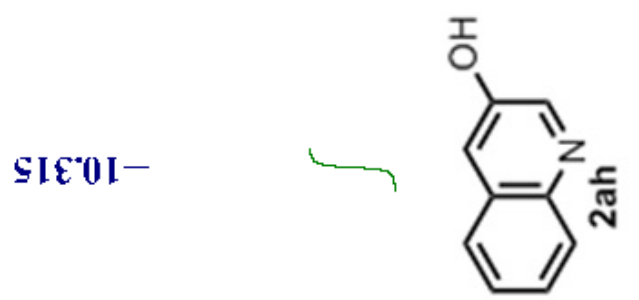

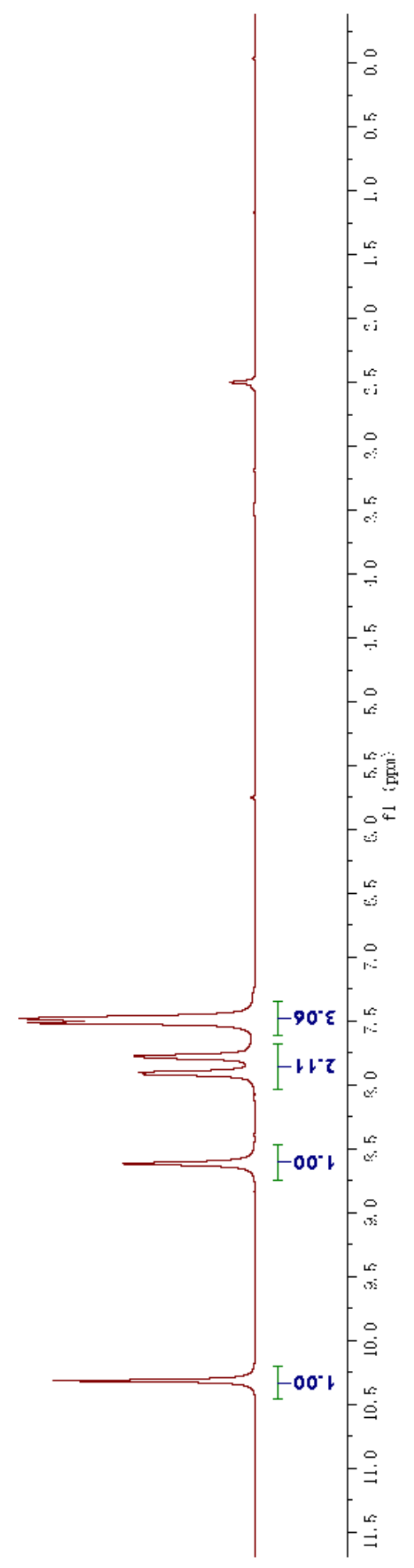




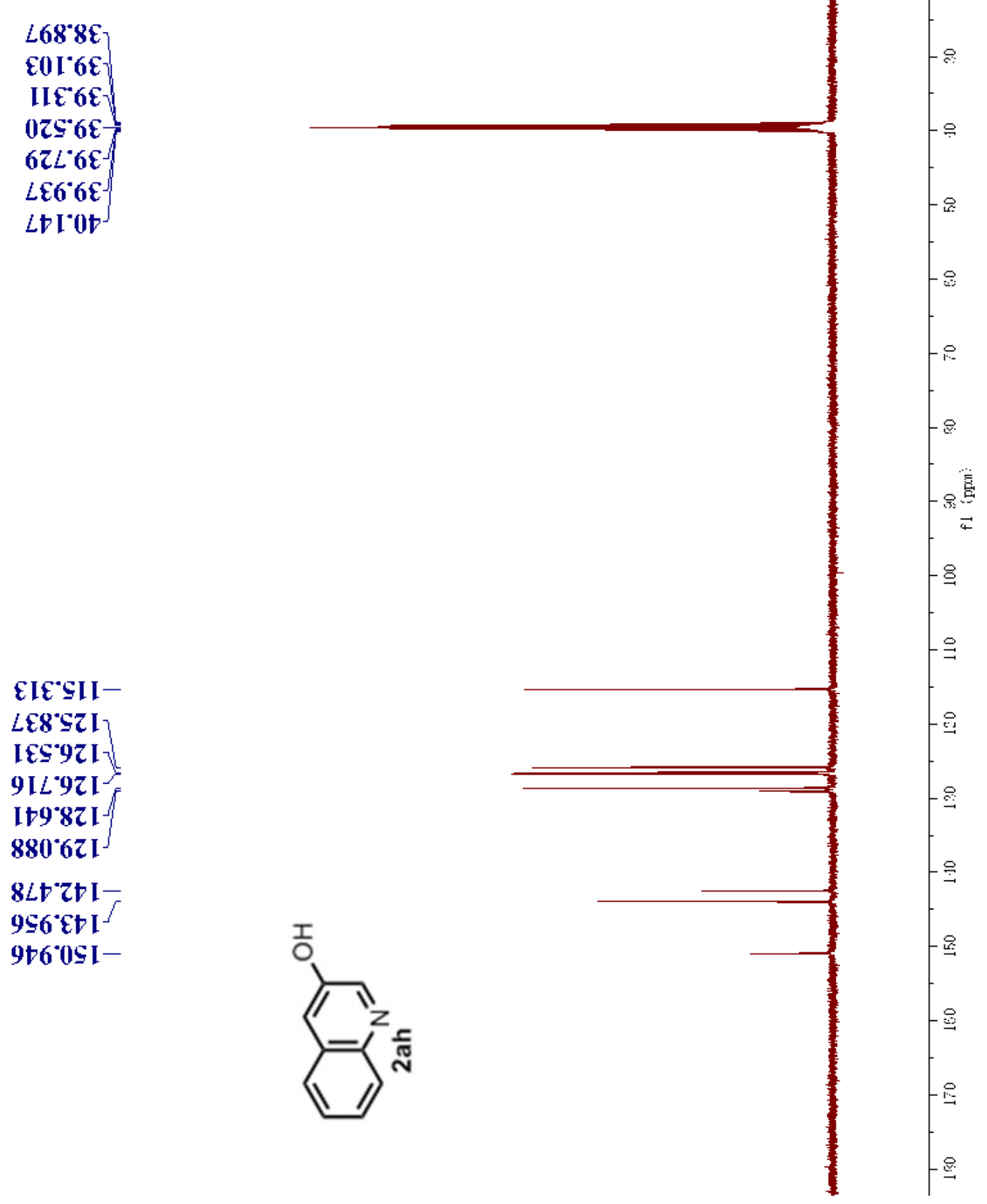




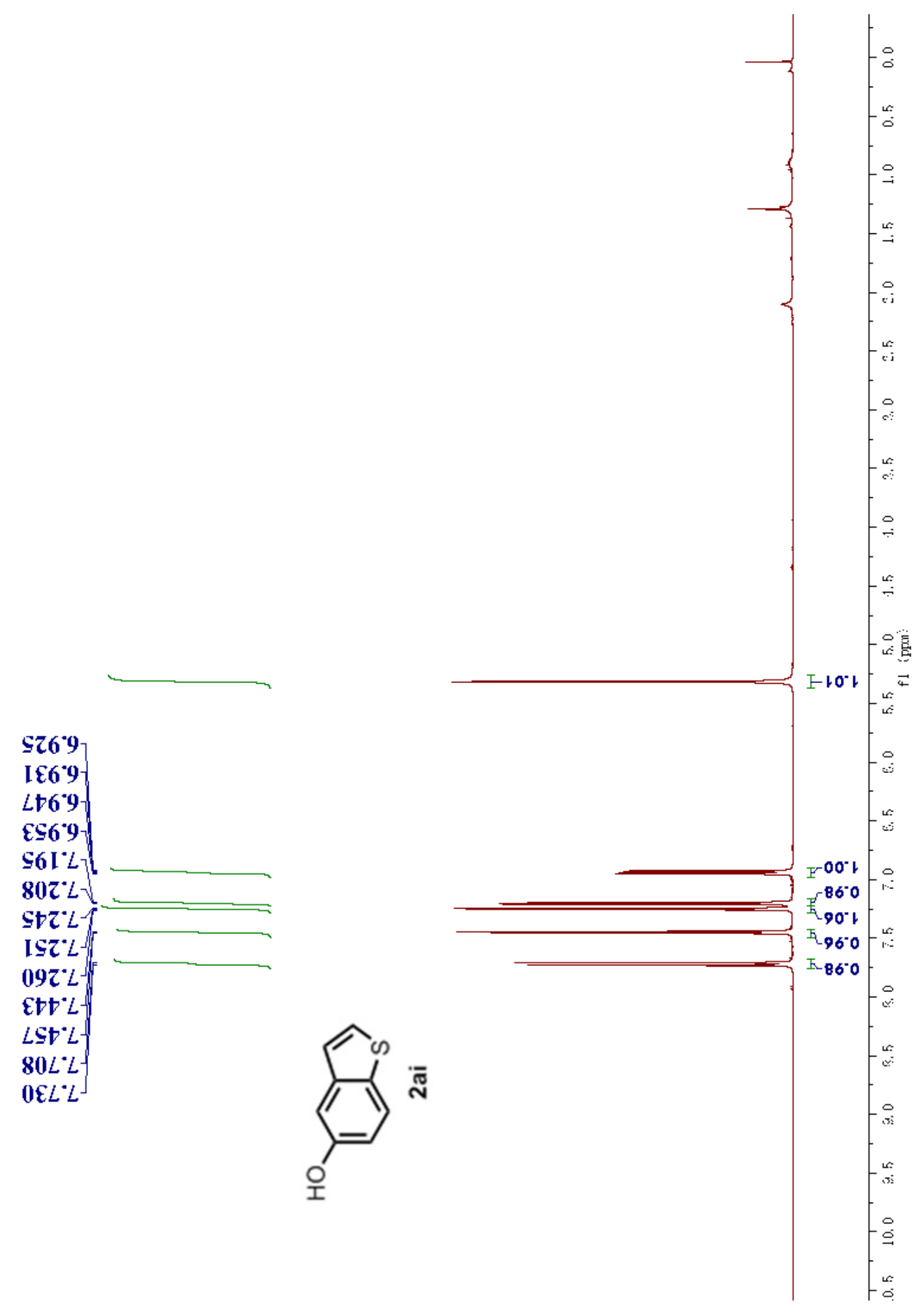



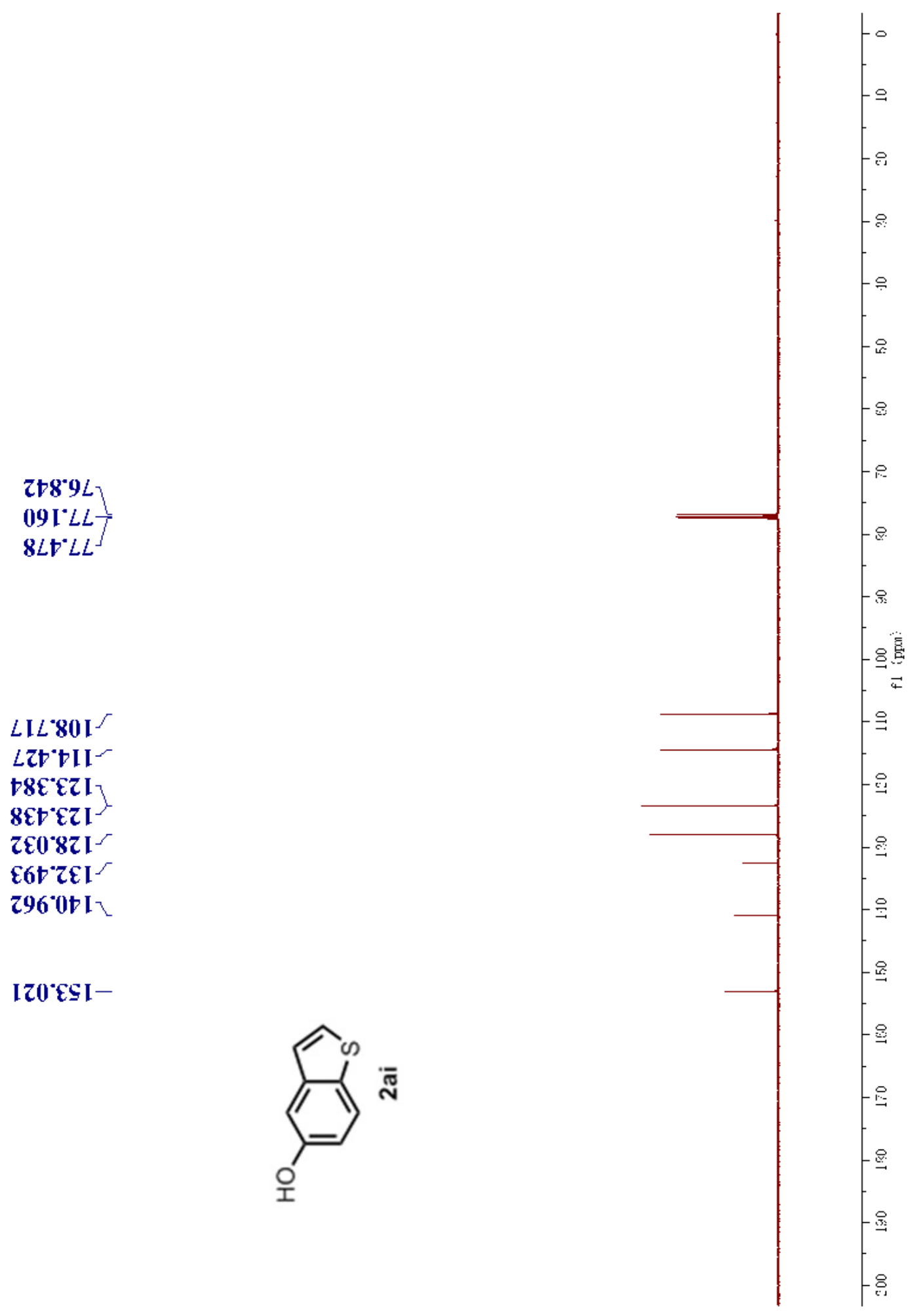


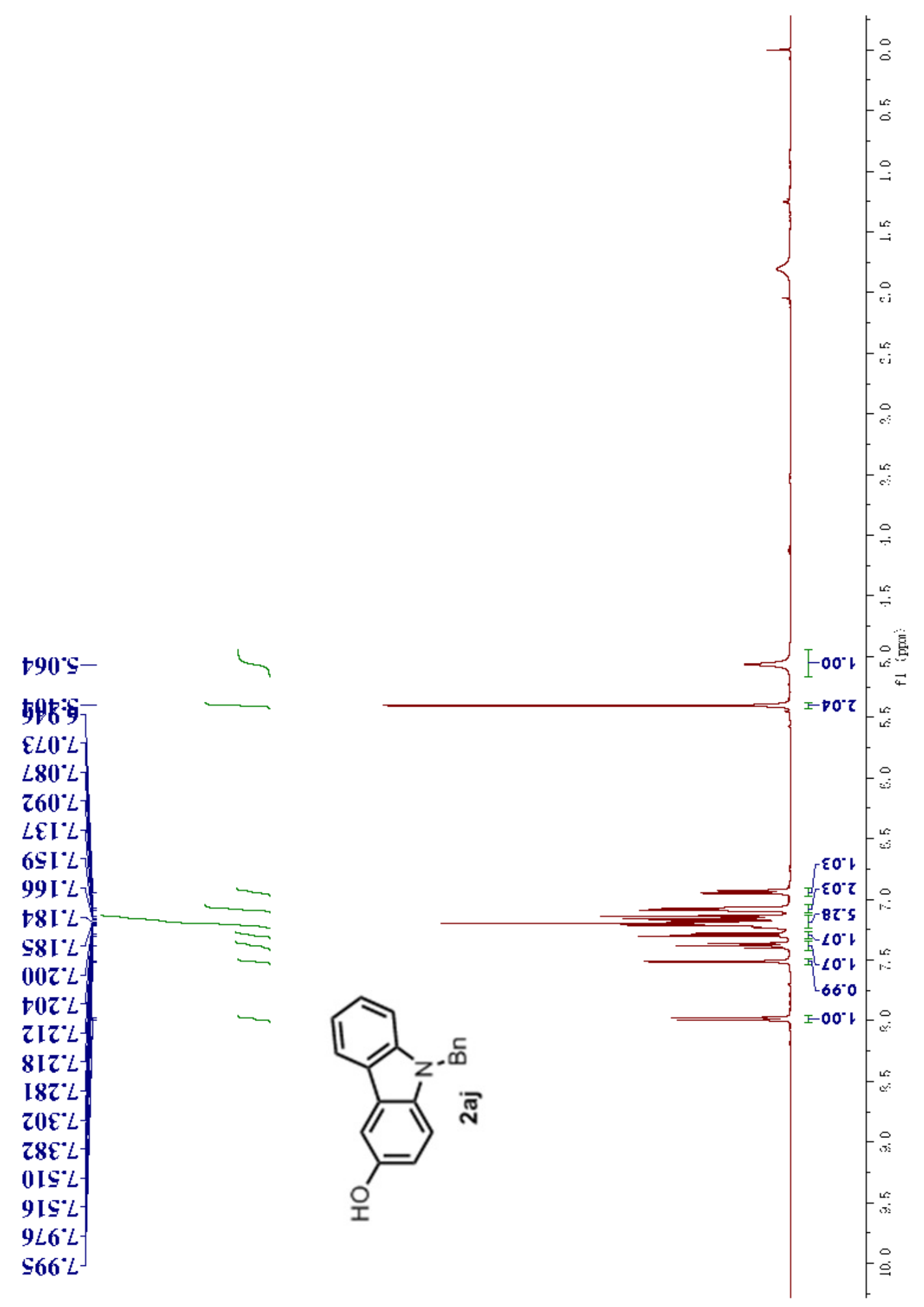




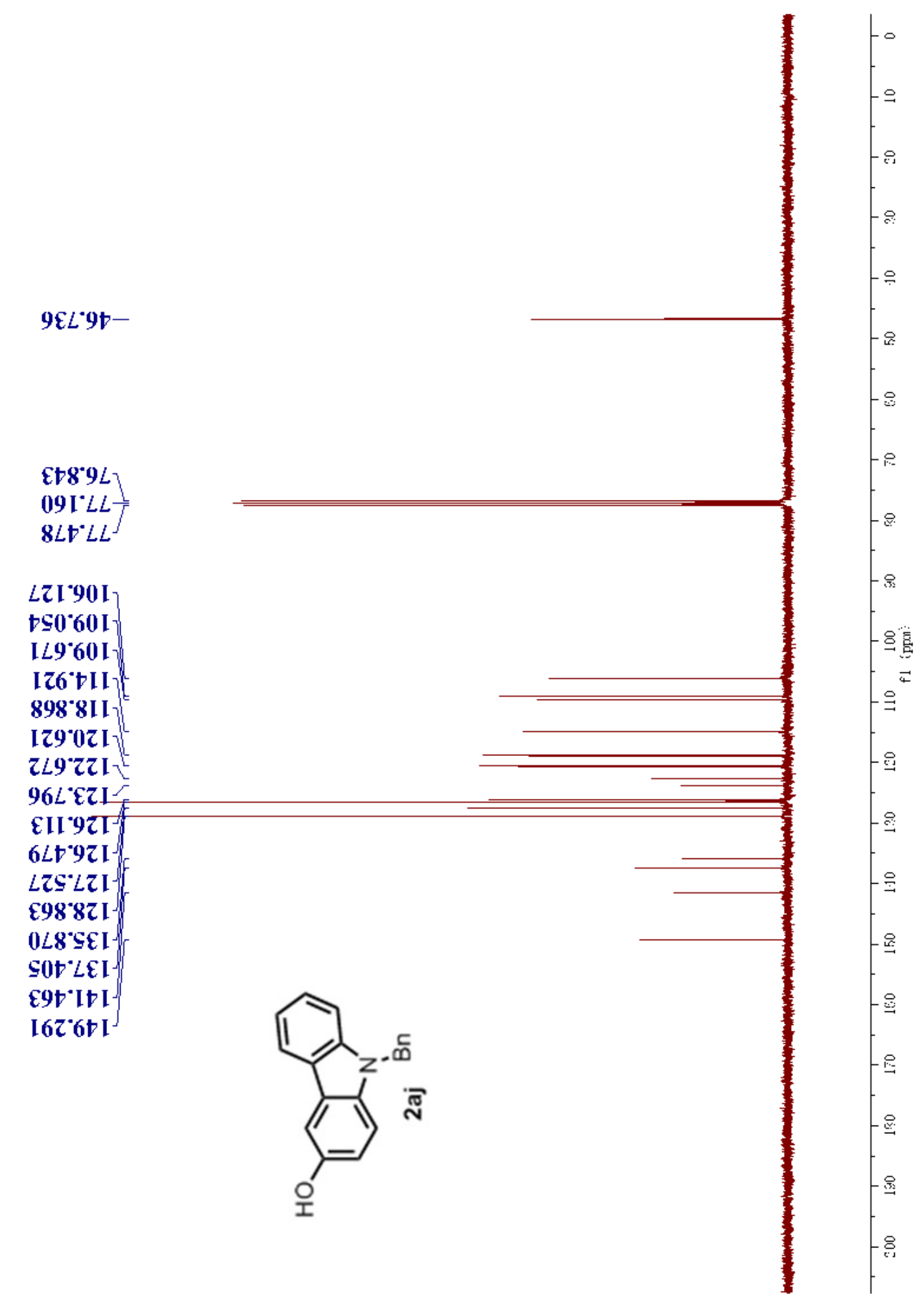




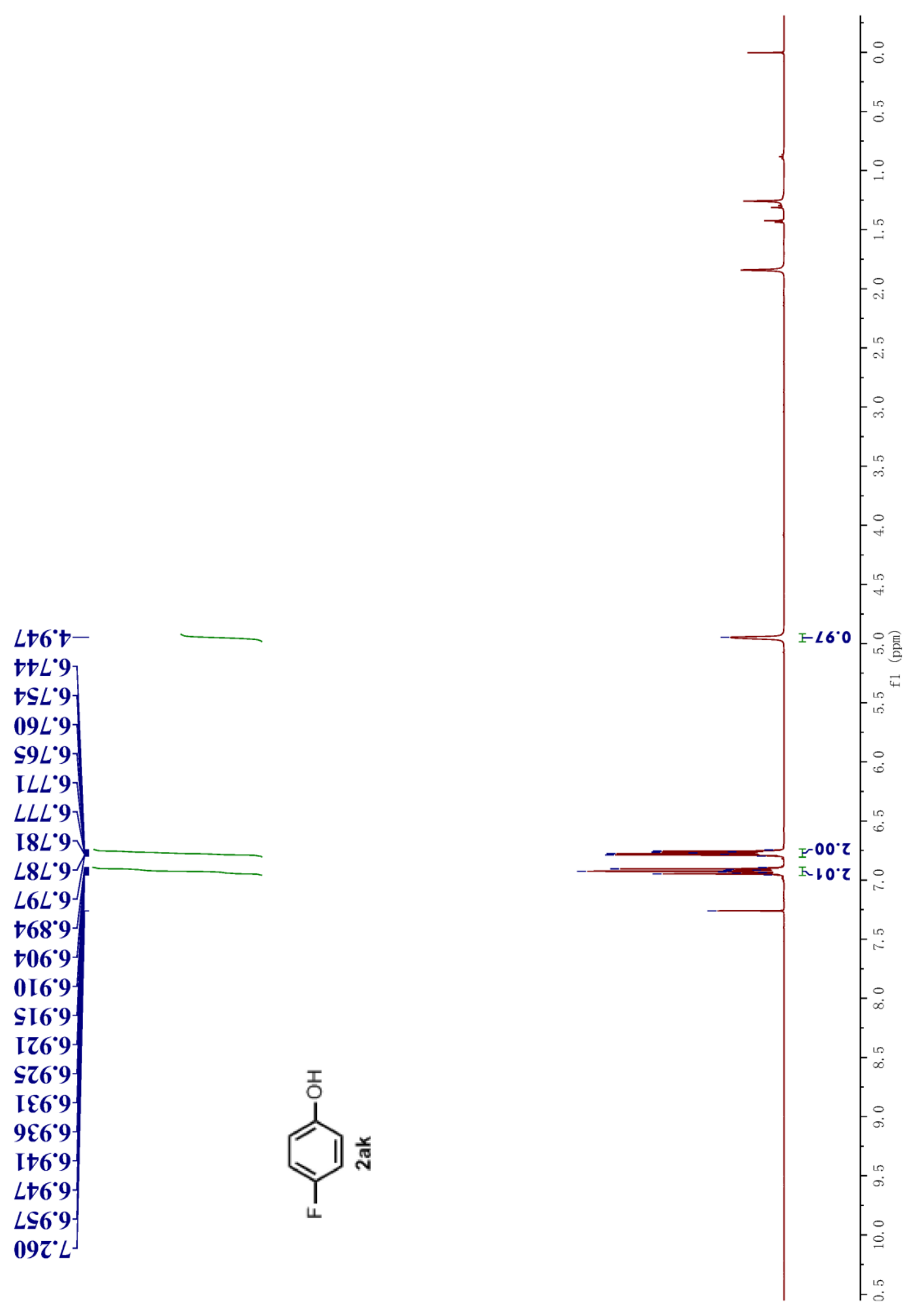




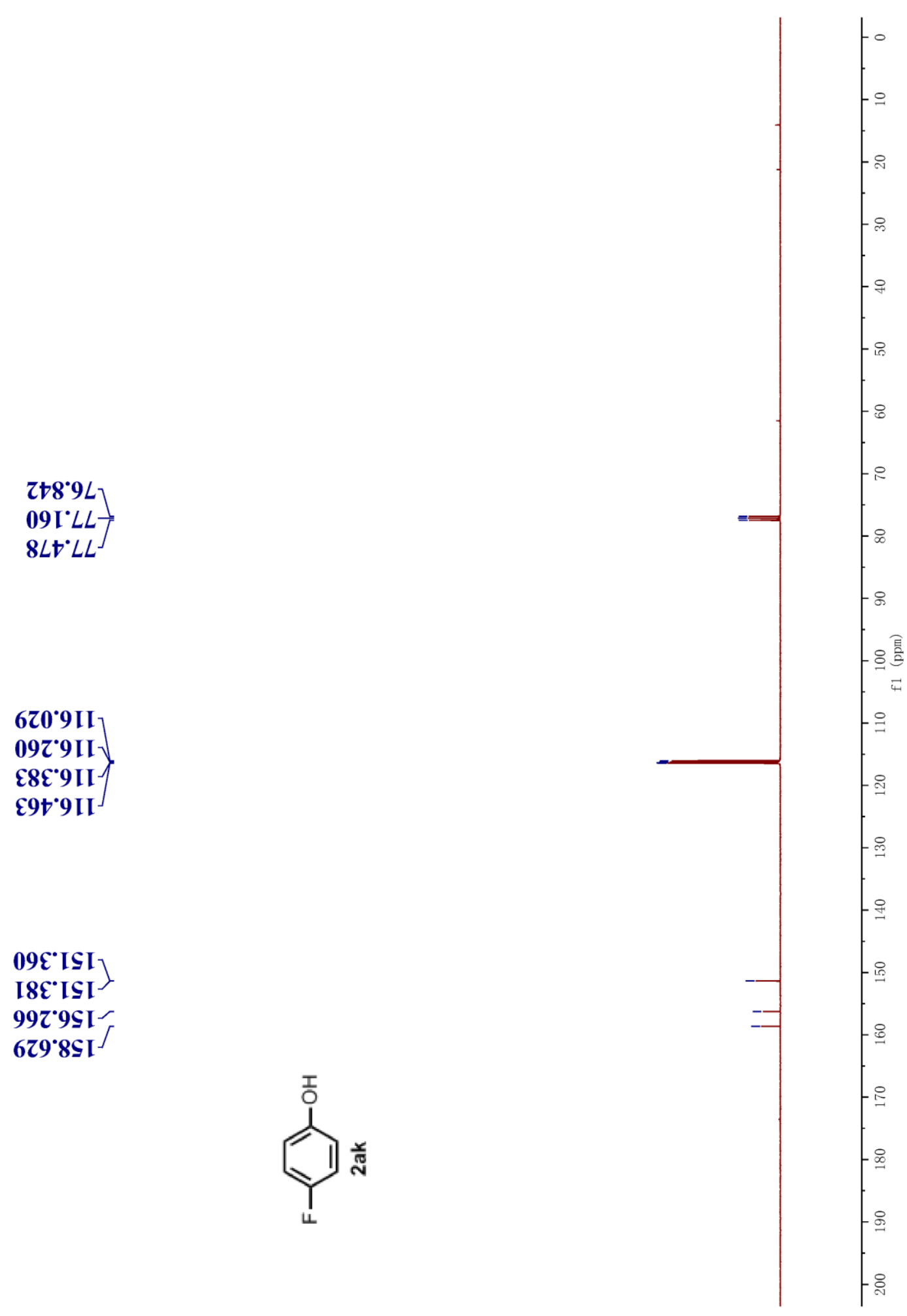




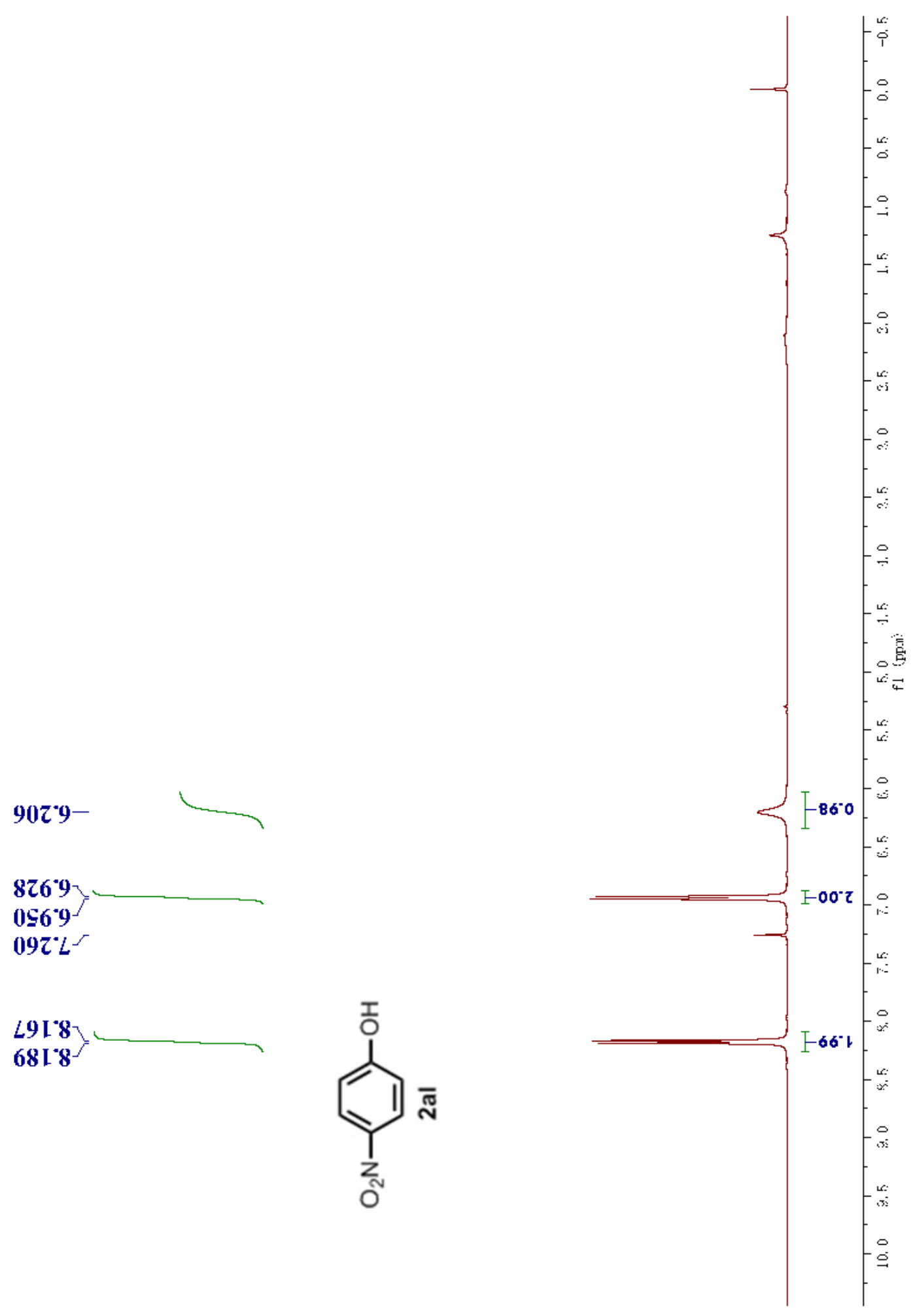




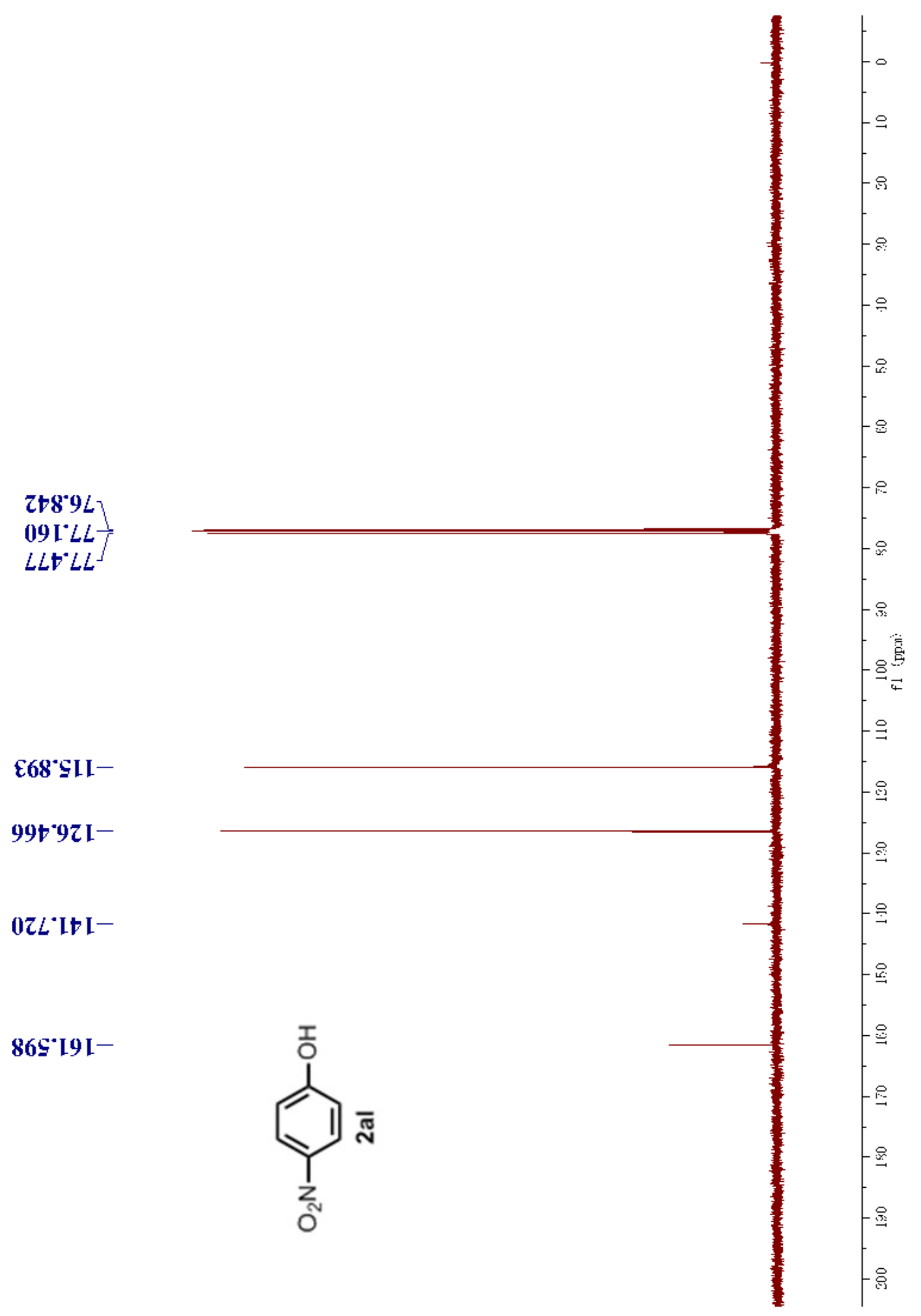




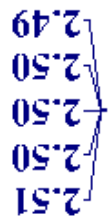

$S L^{*} 9$

$9 L^{\circ} 9$

$9 L^{\circ} 9$

$9 L^{\circ} 9$

$L L^{\circ} 9$

$8 L^{\circ} 9$

$8 L^{\circ} 9$

$8 L^{\circ} 9$

$\varepsilon 6^{\circ} 9$

$56^{\circ} 9$

56.9

$56^{\circ} 9$

$\$ 6^{\circ} 9$

$\$ 6^{\circ} 9^{-}$

$96^{\circ} 9$

$96^{\circ} 9$

$0 \mathrm{I}^{\circ} \mathrm{L}$

II $^{\circ} L$

ZI $L$

$\varepsilon I^{\circ} L$

$\varepsilon I^{\circ} L$

$\rightarrow I^{\circ} L$

$580^{\circ}-$

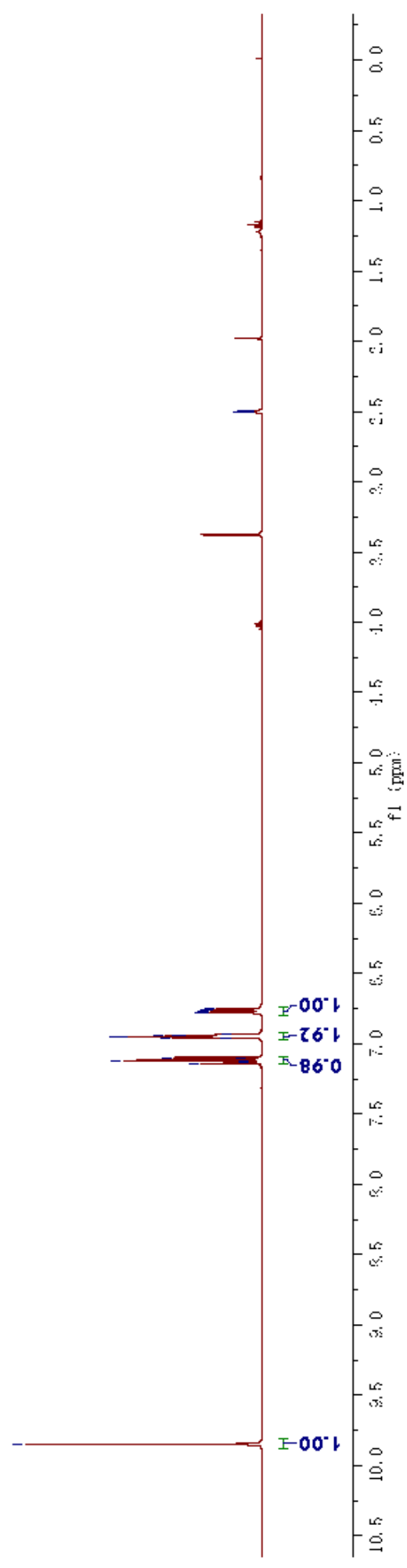




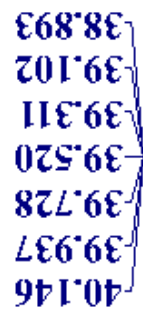

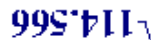

LII'8II

St9.IZI

LI8 IZI

8ZI'IEI-

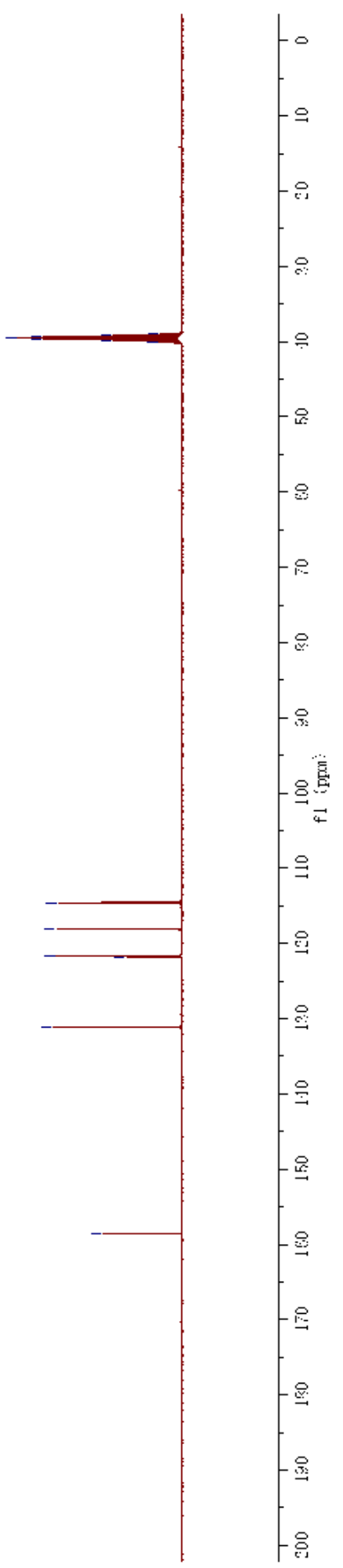




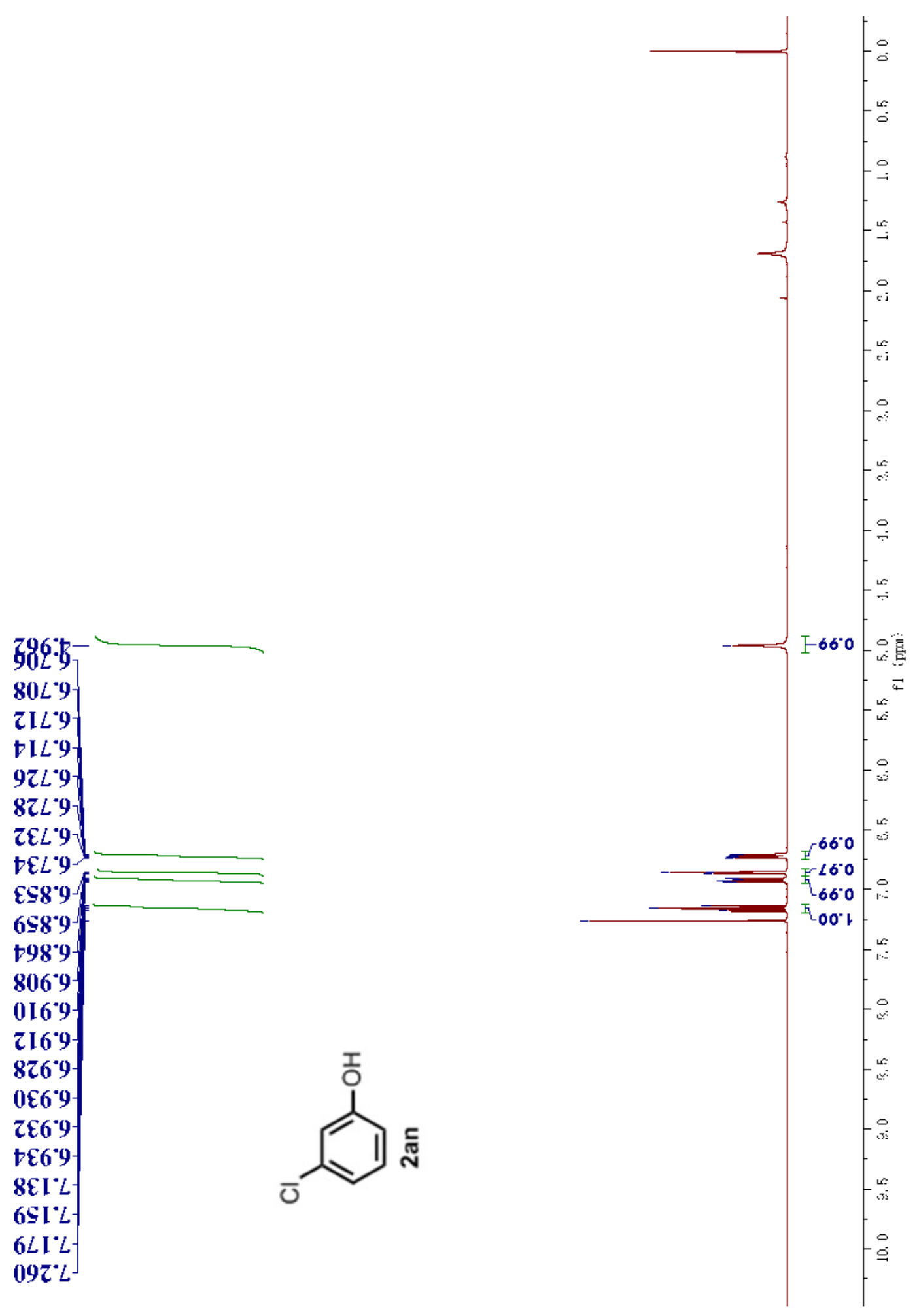




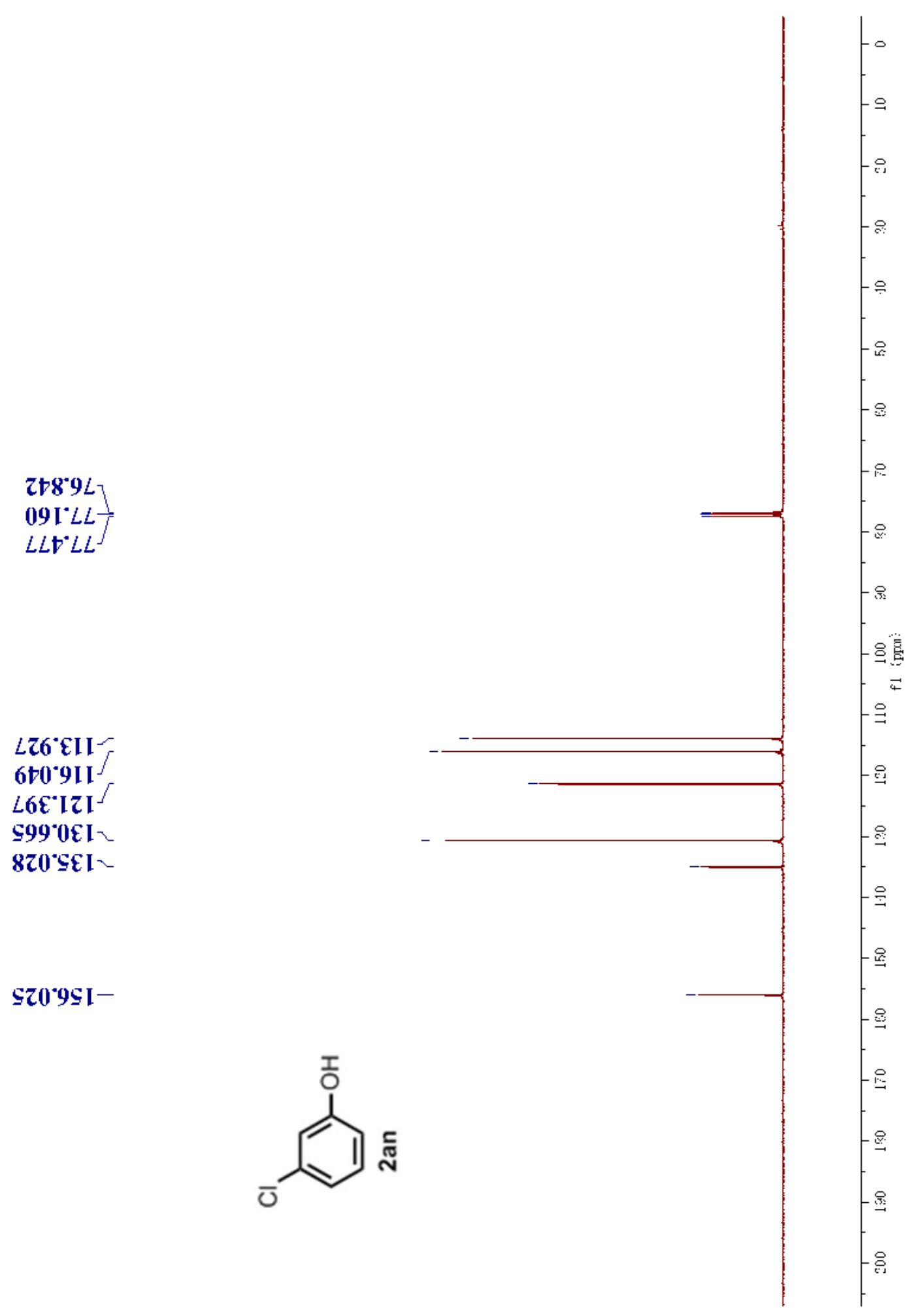


$\varepsilon L L^{*} L$

$26 \mathrm{I}^{\circ} \mathrm{L}$

0 I $\sigma^{\circ} L$

$09 \tau^{\circ} L$

$\angle t E L$

ISE $L$

$89 E L$

ZLE $L$

$\angle 8 E^{\circ} L$

$86 E^{\circ} L$

$806^{\circ} L$

$6 \mathrm{Lt} L$

Zzt $L$

$\angle Z t^{\circ} L$

$\angle 0 L L$

$92 L^{\circ} L^{\prime}$

$62 L L$

$t Z L^{\circ} 8$

$82 L^{\circ} 8$

$\subseteq E L^{\circ} 8$

$6 E L^{\circ} 8$

$6266^{\circ}$

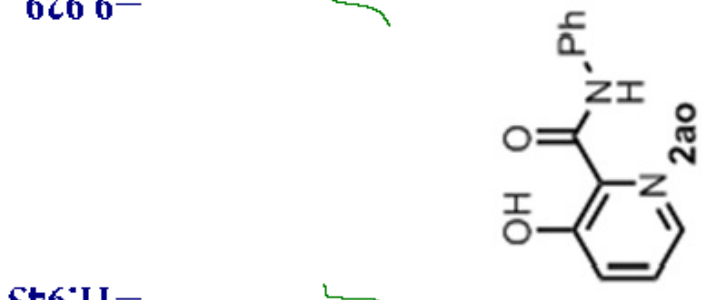

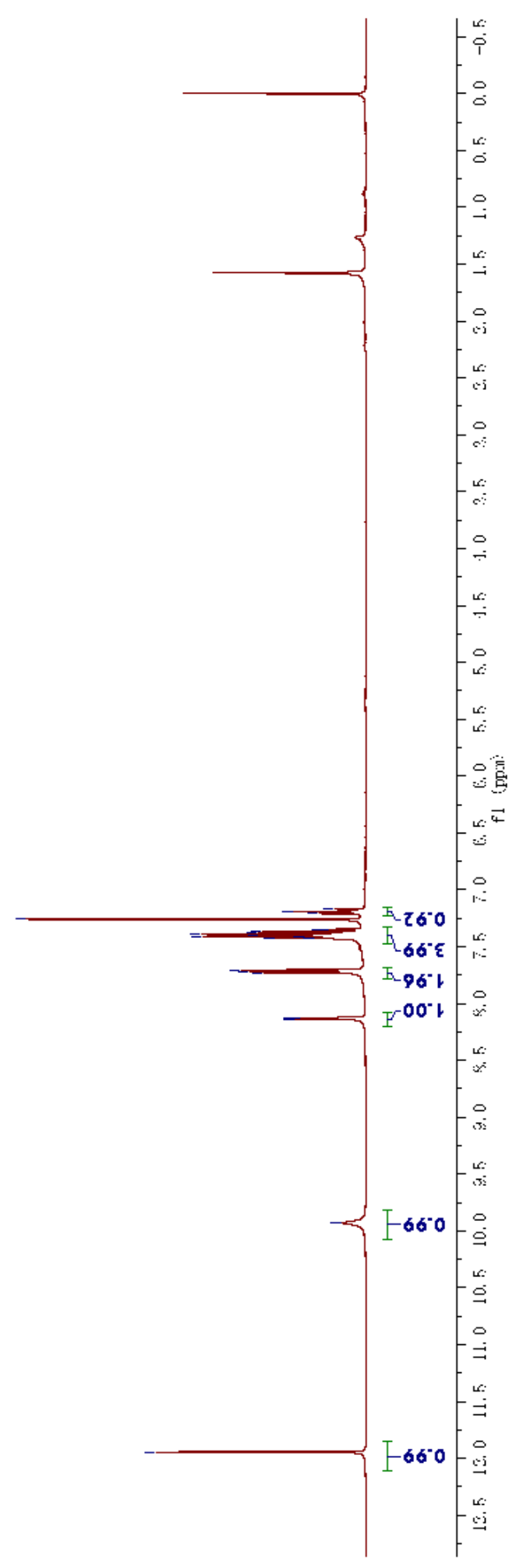


$2+8^{\circ} 9 L$
$09 L^{\circ} L L \frac{1}{5}$
$8 L 6^{\circ} L L$

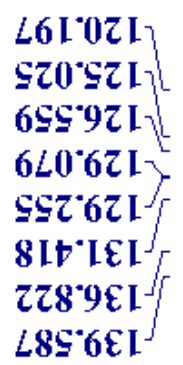

$9 S E^{\circ} 8 \mathrm{I}-$

$\varepsilon 26^{\circ 99} \mathrm{I}^{-}$

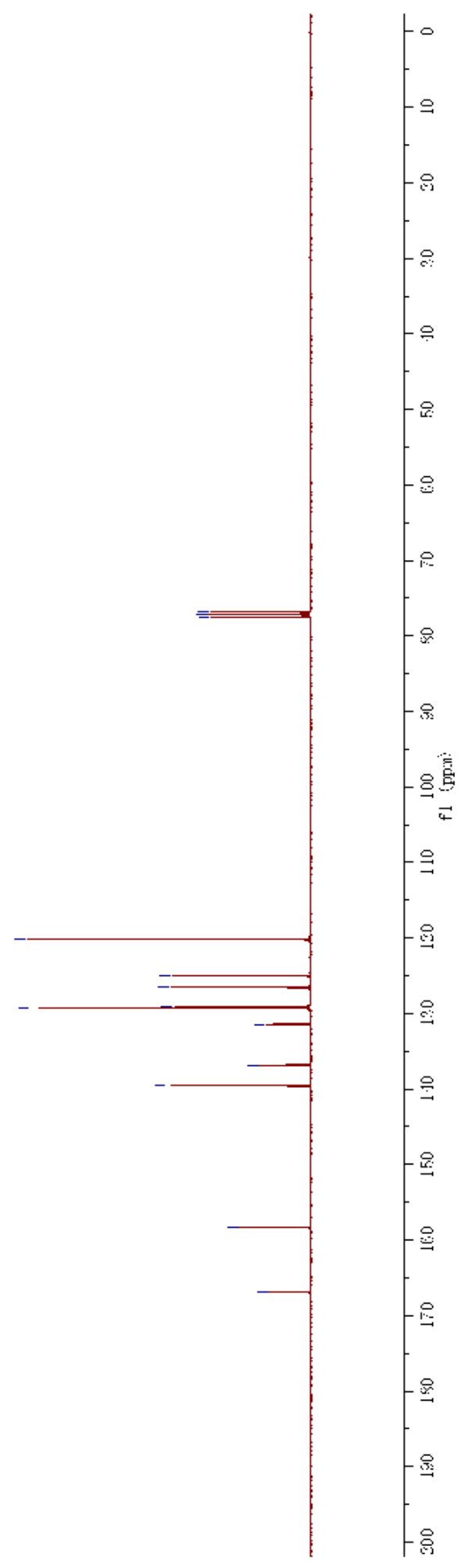




$$
\left.\begin{array}{l}
005 z \\
b 05 z \\
605 z \\
\varepsilon \text { IS } z \\
8 I S z
\end{array}\right]
$$

$900^{\circ} \mathrm{-}-$
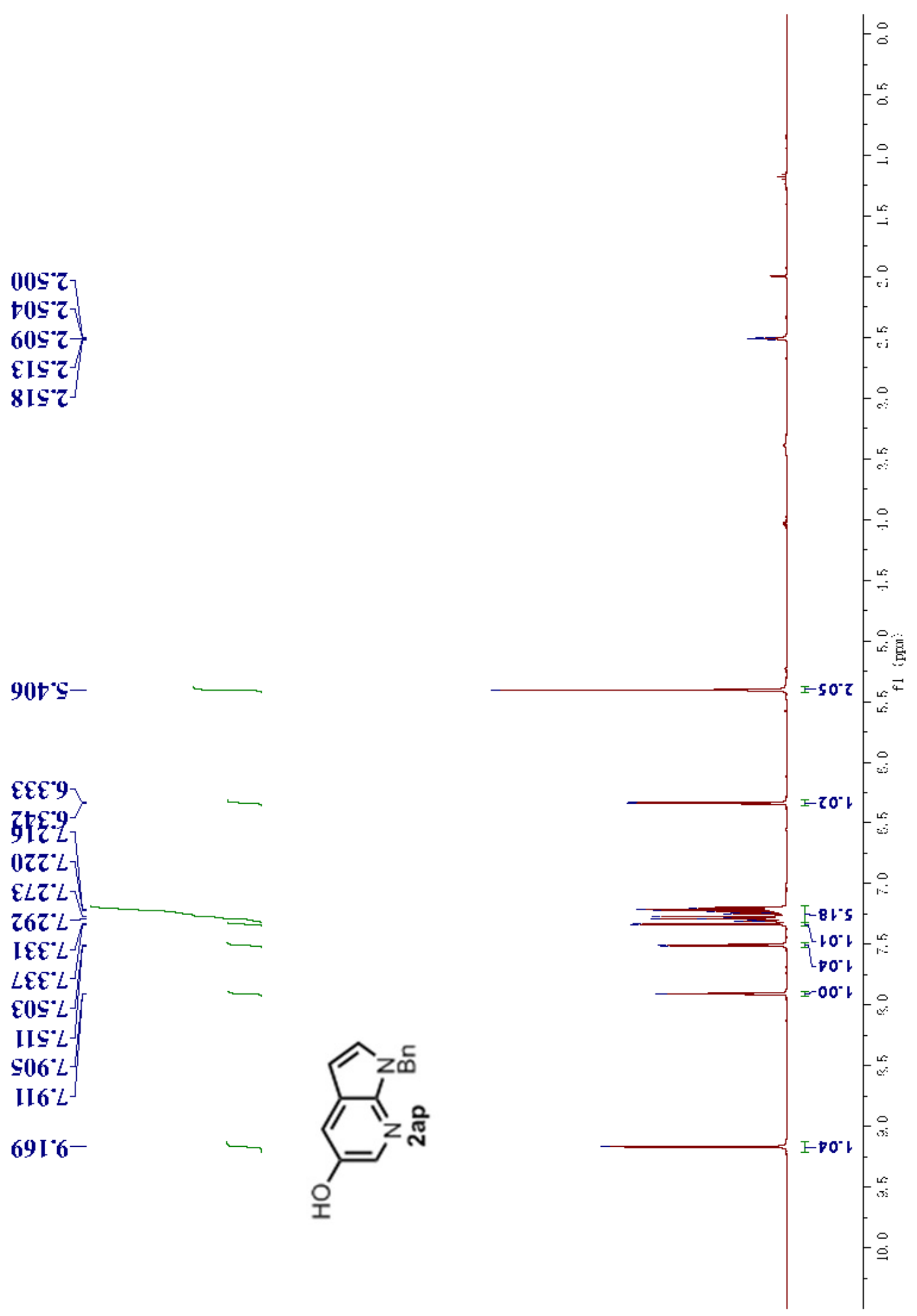


$$
\begin{aligned}
& 768^{\circ} 8 \varepsilon_{7} \\
& \text { 20I'6E } \\
& \text { IIE } 6 \varepsilon \\
& \text { 0ZS" } 6 \varepsilon \\
& 8 z L^{\circ} 6 \varepsilon \\
& \angle \mathcal{E} 6^{\circ} 6 \mathcal{E} \\
& 9 \mathrm{t} \mathbf{I}^{\circ} \mathrm{Ot} \\
& \text { I } \varepsilon Z^{\circ} \angle t
\end{aligned}
$$

$\$ s t \times 6-$
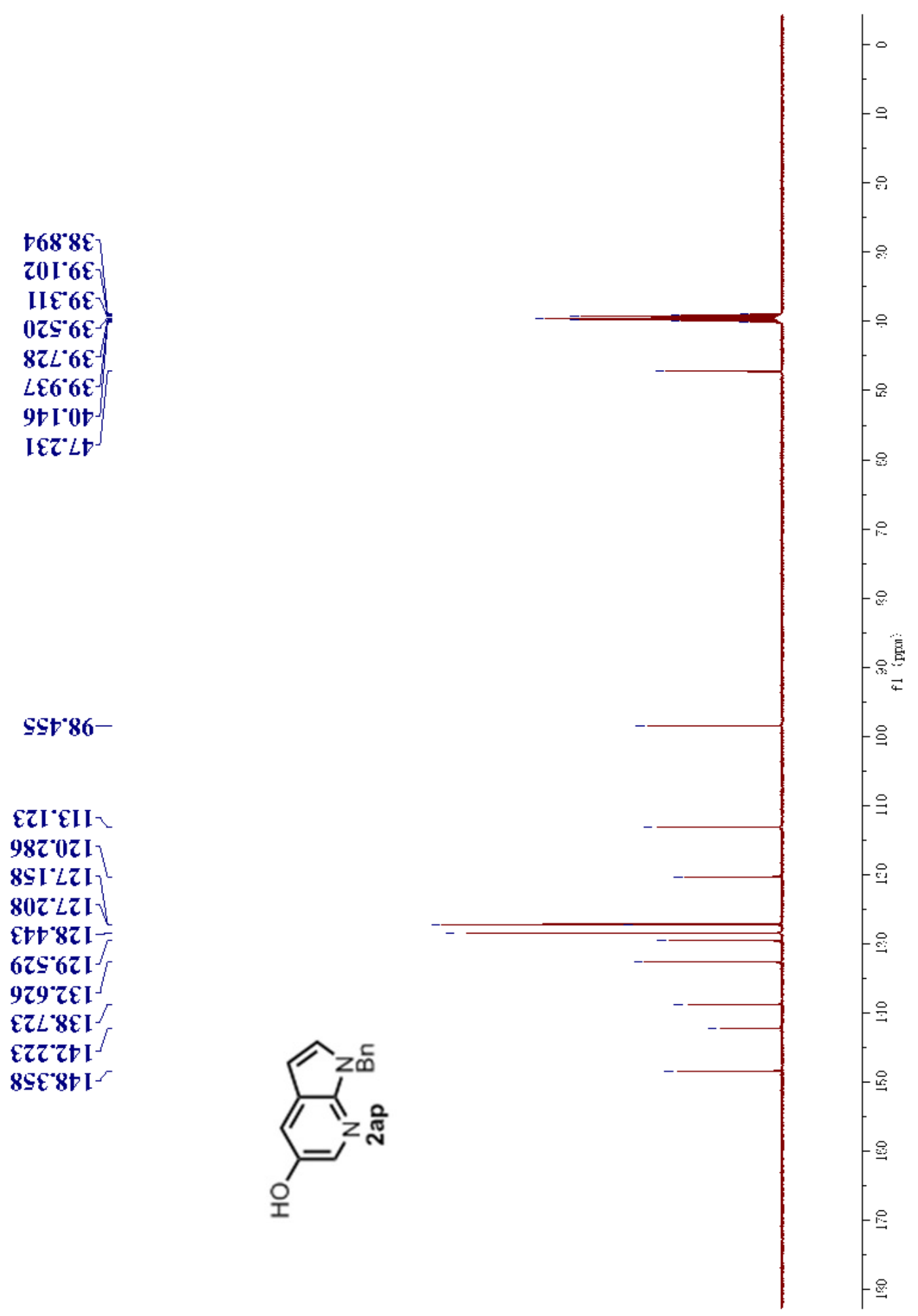